$\underline{\text { Preprint typeset in JHEP style - HYPER VERSION }}$

arXiv:1110.nnnn [hep-th]

\title{
Holographic Calculations of Rényi Entropy
}

\author{
Janet Hung, ${ }^{1}$ Robert C. Myers, ${ }^{1}$ Michael Smolkin ${ }^{1}$ and Alexandre Yale ${ }^{1,2}$ \\ ${ }^{1}$ Perimeter Institute for Theoretical Physics, \\ Waterloo, Ontario N2L 2Y5, Canada \\ ${ }^{2}$ Department of Physics 8 Astronomy and Guelph-Waterloo Physics Institute, \\ University of Waterloo, Waterloo, Ontario N2L 3G1, Canada
}

\begin{abstract}
We extend the approach of [9] to a new calculation of the Rényi entropy of a general CFT in $d$ dimensions with a spherical entangling surface, in terms of certain thermal partition functions. We apply this approach to calculate the Rényi entropy in various holographic models. Our results indicate that in general, the Rényi entropy will be a complicated nonlinear function of the central charges and other parameters which characterize the CFT. We also exhibit the relation between this new thermal calculation and a conventional calculation of the Rényi entropy where a twist operator is inserted on the spherical entangling surface. The latter insight also allows us to calculate the scaling dimension of the twist operators in the holographic models.
\end{abstract}




\section{Contents}

1. Introduction 1

2. Holographic Rényi Entropy 5

2.1 Einstein gravity in any dimension 9

2.2 Gauss-Bonnet gravity and $d \geq 4$

2.3 Quasi-topological gravity and $d=4 \quad[19$

3. Twist Operators and Regulator Surfaces 24

3.1 Twist operators in $d=2$

3.2 Holographic calculation for $d=2$

3.3 UV regulator geometry 32

4. Twist operators and Thermal ensembles 33

4.1 Twist operators and Black holes $\overline{36}$

5. Eigenvalue Spectrum

6. Discussion

A. Rényi entropy inequalities 5

B. Rényi entropy in $d=3$

Q. The on-shell bulk action for $d=2$

\section{Introduction}

Entanglement entropy has emerged as a useful measure of entanglement with which one can characterize various quantum systems e.g., [1], 2, 3]. Given a subsystem $V$ described by a density matrix $\rho_{V}$, the entanglement entropy corresponds to the von Neumann entropy of the corresponding density matrix: $S_{\mathrm{EE}}=-\operatorname{tr}\left[\rho_{V} \log \rho_{V}\right]$. An approach for calculating entanglement entropy for the boundary field theory in gauge/gravity duality was proposed some five years ago in [4] - see also [5, [6]. Given a particular holographic 
framework, the entanglement entropy in the $d$-dimensional boundary theory between a spatial region $V$ and its complement $\bar{V}$ is calculated by extremizing the following expression

$$
S(V)=\frac{2 \pi}{\ell_{\mathrm{P}}^{d-1}} \operatorname{ext}_{v \sim V}^{\operatorname{ext}}[A(v)]
$$

over $(d-1)$-dimensional surfaces $v$ in the bulk spacetime which are homologous to the boundary region $V .{ }^{1}$ In particular then, the boundary of $v$ matches the 'entangling surface' $\partial V$ in the boundary geometry. Implicitly, eq. (1.1) assumes that the bulk theory is well approximated by classical Einstein gravity, where we have adopted the convention: $\ell_{\mathrm{P}}^{d-1}=8 \pi G_{\mathrm{N}}$. Hence this expression (1.1) bears a striking resemblance to black hole entropy, however, the surfaces $v$ do not coincide with any event horizon in general. While there is a good amount of evidence supporting this proposal, e.g., see [5, 7, 8], a general derivation of eq. (1.1) remains lacking. However, we note that a derivation was recently constructed for the special case of a spherical entangling surface in [9].

Another interesting measure of entanglement is the Rényi entropy 10, 11]:

$$
S_{q}=\frac{1}{1-q} \log \operatorname{tr}\left[\rho_{V}^{q}\right] .
$$

where $q$ is a positive real number. Given this expression for the Rényi entropy, there are an number of interesting limits which one may consider: In particular, one finds that the entanglement entropy is recovered in the limit $q$ tends to one, i.e., $S_{\mathrm{EE}}=\lim _{q \rightarrow 1} S_{q}$. Another interesting case to consider is $q \rightarrow \infty$, which yields

$$
S_{\infty}=\lim _{q \rightarrow \infty} S_{q}=-\log \lambda_{1}
$$

where $\lambda_{1}$ is the largest eigenvalue of $\rho_{V}$. This infinite limit is sometimes referred to as the 'min-entropy'. Finally we note that

$$
S_{0}=\lim _{q \rightarrow 0} S_{q}=\log [\mathcal{D}]
$$

where $\mathcal{D}$ corresponds to the number of nonvanishing eigenvalues of $\rho_{V}$. For twodimensional CFT's, there is a universal result for the Rényi entropy of an interval of length $\ell$ [2, 3]:

$$
S_{q}(d=2)=\frac{c}{6}\left(1+\frac{1}{q}\right) \log (\ell / \delta),
$$

\footnotetext{
${ }^{1}$ Hence 'area' $A(v)$ to denotes the $(d-1)$-dimensional volume of $v$. Note that the extremal surface only yields the minimal area if one first Wick rotates to Euclidean signature.
} 
where $c$ is the central charge and $\delta$ is a short-distance regulator, in the underlying CFT. Recently, Rényi entropy has drawn some attention in the condensed matter community [12]. Essentially, all of these results rely on an approach to calculating Rényi entropy using the 'replica trick,' which requires evaluating the partition function on a $q$-fold cover of the original background geometry. In fact, this approach combined with the limit $q \rightarrow 1$ is regarded as the standard calculation of entanglement entropy. Now holography geometrizes calculations in the boundary theory and so a natural holographic implementation of the replica trick involves a singular bulk geometry, reflecting the singular nature of the $q$-fold cover in the boundary [13]. However, attempting to work with this singular bulk space in a straightforward way 13 produces incorrect results [7]. Hence it seems that without a full understanding of string theory or quantum gravity in the bulk, one will not be able to work with such singular bulk space in a controlled way. This problem then stands as an obstacle both to producing a derivation of the holographic expression of entanglement entropy (1.1) and to performing holographic calculations of Rényi entropy (1.2). However, one can also imagine finding a new solution for the bulk geometry which remains smooth despite the singularity in the boundary metric [7, 14]. Essentially our calculations below follow this route.

Recently, ref. [9] presented a new calculation of entanglement entropy of a CFT for a spherical entangling surface in flat $d$-dimensional Minkowski space, ${ }^{2}$ which has a simple holographic translation. One first considers the causal development of the ball enclosed by the entangling surface and then observes that this spacetime region can be conformally mapped to a 'hyperbolic cylinder,' $R \times H^{d-1}$. The curvature scale on the hyperbolic spatial slices $H^{d-1}$ matches the radius of the original spherical entangling surface, $R$. Further the vacuum of the CFT in the original spacetime is mapped to a thermal bath in the hyperbolic cylinder with temperature

$$
T_{0}=\frac{1}{2 \pi R}
$$

As with any other operator in the CFT, the density matrix in the new spacetime $R \times H^{d-1}$ is related to that in the original geometry by a unitary transformation, i.e., $\rho_{V}=U^{-1} \rho_{\text {therm }} U$. Hence the entanglement entropy for the spherical entangling surface is equivalent to the thermal entropy of the CFT at temperature $T_{0}$ in the hyperbolic cylinder $R \times H^{d-1}$. While this insight may not be particularly useful for a generic CFT, using the AdS/CFT correspondence for a holographic CFT, we can relate the latter thermal bath to an appropriate black hole in the bulk AdS space. Given the geometry of $R \times H^{d-1}$, it is natural the thermal bath in the boundary theory would be described

\footnotetext{
${ }^{2}$ This construction is also easily extended to a spherical entangling surface in a 'cylindrical' background with the topology $R \times S^{d-1}[9]$.
} 
by a 'topological' black hole, for which the event horizon has a hyperbolic cross-section [15]. In fact, the dual black hole with temperature $T_{0}$ is simply a particular foliation of empty AdS spacetime with slices which are topologically equivalent to $R \times H^{d-1}$. Here the coordinate transformation in the bulk from Poincaré coordinates to the hyperbolic foliation implements the conformal transformation described above on the boundary. ${ }^{3}$ In any event, in this holographic framework, the entanglement entropy is now given by the horizon entropy of this black hole, which is easily calculated using Wald's formula [16] for any general gravitational theory in the bulk.

Now we observe this derivation presented in [9] also readily lends itself to a holographic calculation of Rényi entropy for a spherical entangling surface. Following the above construction, the density matrix is (essentially) thermal and we can write the $q$ 'th power of $\rho_{V}$ as

$$
\rho_{V}^{q}=U^{-1} \frac{\exp \left[-q H / T_{0}\right]}{Z\left(T_{0}\right)^{q}} U \text { where } Z\left(T_{0}\right) \equiv \operatorname{tr}\left[e^{-H / T_{0}}\right] .
$$

The unitary transformation $U$ and its inverse, appearing above, will cancel upon taking the trace of this expression. Hence we are left with

$$
\operatorname{tr}\left[\rho_{V}^{q}\right]=\frac{Z\left(T_{0} / q\right)}{Z\left(T_{0}\right)^{q}}
$$

Now using the usual definition of the free energy, i.e., $F(T)=-T \log Z(T)$, the corresponding Rényi entropy (1.2) becomes

$$
S_{q}=\frac{q}{1-q} \frac{1}{T_{0}}\left[F\left(T_{0}\right)-F\left(T_{0} / q\right)\right] .
$$

We note that this result matches that in [17, which examined Rényi entropies for a thermal ensemble. Further using the standard thermodynamic identity, $S=-\partial F / \partial T$, we can rewrite this expression as

$$
S_{q}=\frac{q}{q-1} \frac{1}{T_{0}} \int_{T_{0} / q}^{T_{0}} S_{\text {therm }}(T) d T
$$

where $S_{q}$ is the desired Rényi entropy while $S_{\text {therm }}(T)$ denotes the thermal entropy of the CFT on $R \times H^{d-1}$. Eq. (1.10) will be convenient for our holographic calculations in the following. It is clear from this expression that we recover the desired result for the entanglement entropy

$$
S_{\mathrm{EE}}=\lim _{q \rightarrow 1} S_{q}=S_{\mathrm{therm}}\left(T_{0}\right) .
$$

\footnotetext{
${ }^{3}$ The interested reader will find the precise details of this transformation of the bulk coordinates in [9].
} 
with $T_{0}$ given in eq. (1.6).

This discussion demonstrates that for any CFT, Rényi entropies of a spherical entangling surface can be calculated if the thermal entropy on the hyperbolic cylinder $R \times H^{d-1}$ is known for an arbitrary temperature. As with the entanglement entropy, this approach will now allow us to perform holographic calculations of the Rényi entropy of the boundary CFT in the case where the entangling surface is a sphere $S^{d-1}$. The essential new ingredient is that we must understand the thermodynamic properties of the dual topological black holes away from $T=T_{0}$. Hence our analysis of holographic Rényi entropy is restricted to bulk gravitational theories where these black hole solutions are known. Hence the present discussion differs from that of holographic entanglement entropy in [9], which applied for any covariant theory of gravity in the bulk.

An overview of the remainder of the paper is as follows: We begin by explicitly calculating the holographic Rényi entropy as described above for three bulk theories: Einstein gravity, Gauss-Bonnet gravity [18] and quasi-topological gravity [19, 20 in sections 2.1, 2.2 and 2.3, respectively. Our results indicate that the Rényi entropy (and in particular, the universal contribution) is a complicated nonlinear function of the central charges and other parameters which characterize the underlying CFT. In section 3, we review the calculation of Rényi entropy in terms of twist operators for twodimensional CFT's and investigate the holographic representation of these calculations. Section 1 presents the connection between the 'thermal calculation' of Rényi entropy discussed above and the calculation where a twist operator is inserted on the spherical entangling surface. This discussion applies for CFT's in any dimension and so in section 4.1, we apply these insights to calculate the scaling dimension of the twist operators for the holographic models introduced in section 2. Finally, in section 5, we begin to examine what information can be inferred about the eigenvalue spectrum of the reduced density matrix using our results for the Rényi entropy. Then we conclude with a discussion of our results in section 6. A number of appendices are included to cover certain ancillary discussions. in appendix A, we consider various inequalities which Rényi entropy must generally satisfy and their implications for our holographic results.. Appendix B presents a nontrivial holographic model for a three-dimensional boundary CFT. Our calculations with this model indicate that there is no particular simplification of the Rényi entropy for $d=3$. Finally, we present some details of holographic calculations relevant for section 3 in appendix $Q$.

\section{Holographic Rényi Entropy}

As described in the introduction, our goal is to use the AdS/CFT correspondence to cal- 
culate the Rényi entropy for a spherical entangling surface in the boundary conformal field theory. We do this for three bulk gravitational theories of increasing complexity, beginning with Einstein gravity in section 2.1 and then considering Gauss-Bonnet gravity in section 2.2 and quasi-topological gravity in section 2.3 . In appendix B, we also make a perturbative analysis of a four-dimensional bulk theory with an interaction term which is cubic in the Weyl tensor. As we describe below, the additional couplings appearing in these bulk gravity theories allow us to consider a broader class of dual boundary CFT's. The three gravitational theories in this section were chosen because, in each case, a family of analytic solutions is known corresponding to topological black holes with hyperbolic horizons. Using the standard tools of black hole thermodynamics, i.e., the Wald entropy [16, we are then able to calculate the horizon entropy for these black holes. The standard AdS/CFT dictionary equates this horizon entropy to the thermal entropy of the boundary CFT on the hyperbolic cylinder $R \times H^{d-1}$. Hence using eq. (1.10) we can calculate the Rényi entropy for a spherical entangling surface.

Before moving to specific cases, let us consider the gravitational calculations in a little more detail. We are considering a $d$-dimensional boundary theory and hence the bulk spacetime has $d+1$ dimensions. For the three gravity theories of interest, the metric for the topological black holes will take the form

$$
d s^{2}=-\left(\frac{r^{2}}{L^{2}} f(r)-1\right) N^{2} d t^{2}+\frac{d r^{2}}{\frac{r^{2}}{L^{2}} f(r)-1}+r^{2} d \Sigma_{d-1}^{2},
$$

where $d \Sigma_{d-1}^{2}$ is the line element for the $(d-1)$-dimensional hyperbolic plane $H^{d-1}$ with unit curvature. The function $f(r)$ is determined by the field equations of the particular gravity theory under consideration. We will find that asymptotically $f(r \rightarrow \infty) \equiv f_{\infty}$ and hence, from $g_{r r}$, we can see that the AdS curvature scale is given by $\tilde{L}^{2}=L^{2} / f_{\infty}$. We have included an extra constant $N^{2}$ in $g_{t t}$ to allow us to adjust the normalization of the time coordinate. In particular, we will choose $N^{2}=L^{2} /\left(f_{\infty} R^{2}\right)=\tilde{L}^{2} / R^{2}$ to ensure that the boundary metric is conformally equivalent to

$$
d s_{\infty}^{2}=\left(-d t^{2}+R^{2} d \Sigma_{d-1}^{2}\right)
$$

That is, we are studying the boundary CFT on $R \times H^{d-1}$ with the curvature scale of the hyperbolic spatial slices set to $R$.

The position of the event horizon, given by vanishing of $g_{t t}$, is defined by the expression

$$
\frac{r_{\mathrm{H}}^{2}}{L^{2}} f\left(r_{\mathrm{H}}\right)=1 .
$$


The temperature of the thermal bath in the boundary CFT is given by the Hawking temperature of the horizon, which may be written as

$$
\begin{aligned}
T & =\frac{N}{4 \pi} \partial_{r}\left[\frac{r^{2}}{L^{2}} f(r)\right]_{r=r_{\mathrm{H}}} \\
& =\frac{\tilde{L}}{4 \pi R}\left[\frac{2}{r_{\mathrm{H}}}+\left.\frac{r_{\mathrm{H}}^{2}}{L^{2}} \frac{\partial f(r)}{\partial r}\right|_{r=r_{\mathrm{H}}}\right] .
\end{aligned}
$$

At this point, we note that setting $f(r)$ to a constant, i.e., $f(r)=f_{\infty}$, always provides a solution of the gravitational equations of motion. This is the case where the metric (2.1) corresponds to a pure AdS space with a hyperbolic foliation. With this choice, eq. (2.3) yields $r_{\mathrm{H}}=\tilde{L}$ and then from eq. (2.4) we recover the expected temperature as in eq. (1.6), i.e., $T=T_{0}=(2 \pi R)^{-1}$. This AdS metric was central to the discussion of holographic entanglement entropy in 9].

Now one could proceed with a holographic calculation the Rényi entropy using eq. (1.9). In this approach, one would first evaluate the Euclidean gravitational action $I_{\mathrm{E}}$ for the topological black holes (2.1). Then interpreting this result in terms of the free energy of the dual thermal ensemble, i.e., $F(T)=T I_{\mathrm{E}}(T)$, the Rényi entropy is easily calculated with the expression in eq. (1.9). However, we will instead proceed with eq. (1.10) where the thermal entropy is determined as the horizon entropy of the dual black holes. Hence an essential step in our calculations will be evaluating the horizon entropy of these topological black holes (2.1). Since in sections 2.2 and 2.3 we will be considering bulk theories with higher curvature interactions, we calculate the horizon entropy using Wald's formula [16]

$$
S=-2 \pi \int_{\text {horizon }} d^{d-1} x \sqrt{h} \frac{\partial \mathcal{L}}{\partial R_{c d}^{a b}} \hat{\varepsilon}^{a b} \hat{\varepsilon}_{c d}
$$

where $\mathcal{L}$ corresponds to the Lagrangian of the particular gravity theory of interest. We use $\hat{\varepsilon}_{a b}$ to denote the binormal to the horizon.

Again, we interprete this result as the thermal entropy of the boundary CFT at a temperature $T$ given by eq. (2.4) and then we can calculate the Rényi entropy using eq. (1.10). However, rather than considering the entropy as a function of the temperature, it will be more convenient in the following to consider $S(x)$ where $x \equiv r_{\mathrm{H}} / \tilde{L}$. In this case, we can rewrite the expression for the Rényi entropy as

$$
\begin{aligned}
S_{q} & =\frac{q}{q-1} \frac{1}{T_{0}} \int_{x_{q}}^{1} S(x) \frac{d T}{d x} d x \\
& =\frac{q}{q-1} \frac{1}{T_{0}}\left[\left.S(x) T(x)\right|_{x_{q}} ^{1}-\int_{x_{q}}^{1} \frac{d S}{d x} T(x) d x\right]
\end{aligned}
$$


The upper endpoint in the above integral is fixed using $r_{\mathrm{H}}=\tilde{L}$ and hence $x=1$ for $T=T_{0}$. It remains to determine the lower endpoint $x_{q}$ corresponding to the temperature $T=T_{0} / q$. We will see below that the precise value of $x_{q}$ will depend on the details of the gravitational theory under consideration.

The black hole solutions (2.1) above inherit an $S O(1, d-1)$ symmetry from the hyperbolic plane portion of the metric. In particular, the geometry of the horizon is homogenous and the integrand in eq. (2.5) is simply a constant in the cases of interest. Hence we may write the entropy as:

$$
S=-\left.2 \pi r_{\mathrm{H}}^{d-1} \frac{\partial \mathcal{L}}{\partial R^{a b} c d} \hat{\varepsilon}^{a b} \hat{\varepsilon}_{c d}\right|_{r=r_{\mathrm{H}}} V_{\Sigma} .
$$

Here we have introduced $V_{\Sigma}$ to denote the 'coordinate' volume of the hyperbolic plane, i.e., $V_{\Sigma}=\int_{H^{d-1}} d \Sigma$. Of course, this volume is divergent and must be regulated to make sense of the entropy. As described in detail in [9], this divergence is related to the UV divergences appearing in the Rényi entropy.

A convenient choice of coordinates on $H^{d-1}$ is given by ${ }^{4}$

$$
d \Sigma_{d-1}^{2}=\frac{d y^{2}}{y^{2}-1}+\left(y^{2}-1\right) d \Omega_{d-2}^{2},
$$

where $d \Omega_{d-2}^{2}$ is the line-element on a unit $(d-2)$-sphere. As explained in [9], the horizon entropy (2.7) is regulated by integrating out to a maximum radius in this hyperbolic geometry:

$$
y_{\max }=\frac{R}{\delta},
$$

where $\delta$ is the short-distance cut-off in the boundary CFT. Hence the hyperbolic volume becomes

$$
\begin{aligned}
V_{\Sigma} & =\Omega_{d-2} \int_{1}^{y_{\max }}\left(y^{2}-1\right)^{(d-3) / 2} d y \\
& \simeq \frac{\Omega_{d-2}}{d-2}\left[\frac{R^{d-2}}{\delta^{d-2}}-\frac{(d-2)(d-3)}{2(d-4)} \frac{R^{d-4}}{\delta^{d-4}}+\cdots\right],
\end{aligned}
$$

where $\Omega_{d-2}=2 \pi^{(d-1) / 2} / \Gamma((d-1) / 2)$ is the area of a unit $(d-2)$-sphere. Now using the horizon entropy in eq. (2.7) to calculate the Rényi entropy using eq. (1.19), it is clear that the UV divergences appearing in the latter are simply inherited from the above expression for $V_{\Sigma}$.

An important consequence of these calculations is that the UV divergences appearing in the Rényi entropy for any $q$ - and the entanglement entropy given by $q=1$

\footnotetext{
${ }^{4}$ In terms of the coordinates chosen in [9, $y=\cosh u$ or $y=\sqrt{x^{2}+1}$.
} 
- have precisely the same structure. In particular, if we note that the area of the spherical entangling surface is given by $\mathcal{A}_{d-2}=\Omega_{d-2} R^{d-2}$, then from eq. (2.10) the leading divergence in the Rényi entropies will have the form of the 'area law' expected to appear in the entanglement entropy [5, 6]. Note that the hyperbolic geometry of the horizon was essential to ensure the leading power was $1 / \delta^{d-2}$ here despite the area integral being $(d-1)$-dimensional in eq. (2.10). Of course, the power-law divergences in the Rényi entropies are not universal, e.g., see [5, 6], however, a universal contribution can be extracted from the subleading terms. The form of this universal contribution to the entanglement entropy depends on whether $d$ is odd or even [9, 21]:

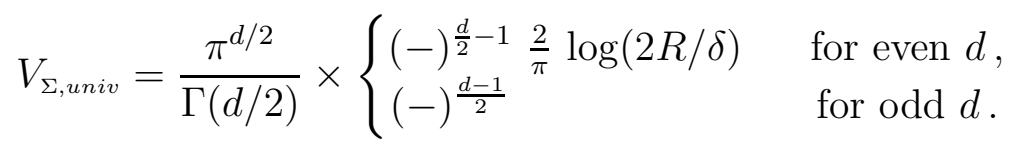

Here we should acknowledge a technical point with regards to odd $d$. As is standard, we have identified here the universal contribution as the constant term appearing in the expansion (2.10). While the universal character of this constant is established in the entanglement entropy, i.e., $S_{1}$, for a variety of $d=3$ conformal quantum critical systems [23], as well as certain three-dimensional (gapped) topological phases [1]. Nevertheless, in general, one may worry whether this constant is affected by the details of scheme chosen to regulate the underlying field theory. However, this issue should be circumvented by considering the mutual information with an appropriate construction [9, 21], as can be explicitly demonstrated in holographic calculations [24]. The potential ambiguity does not appear with the mutual information since the latter is free of any UV divergences - for example, see [25, 26]. We expect that the same approach can be extended to Rényi entropies with general $q$. For the present purposes, however, it will suffice to focus on the above terms (2.11) to identify the universal contribution to the Rényi entropies.

\subsection{Einstein gravity in any dimension}

To begin the detailed calculations, we consider Einstein gravity. Hence the bulk action is simply

$$
I=\frac{1}{2 \ell_{\mathrm{P}}^{d-1}} \int d^{d+1} x \sqrt{-g}\left(\frac{d(d-1)}{L^{2}}+R\right),
$$

and the equations of motion lead to the solution

$$
f(r)=1-\frac{\omega^{d}}{r^{d}}
$$


for the line element in eq. (2.1). Above, $\omega^{d}$ is an integration constant which characterizes the energy (density) of the corresponding black hole. For the present purposes, it is most useful to parameterize this constant in terms of the position of the horizon with

$$
\omega^{d}=r_{\mathrm{H}}^{d}-\tilde{L}^{2} r_{\mathrm{H}}^{d-2} .
$$

Further we note that here, $f_{\infty}=f(r \rightarrow \infty)=1$ and hence $\tilde{L}=L$, i.e., the AdS curvature scale is same as the scale appearing in the cosmological constant term in the action (2.12). The latter equality will not hold for the higher curvature gravity theories in the subsequent sections. Applying eq. (2.4) to the present case, we find that the temperature is given by

$$
T=\frac{1}{4 \pi R}\left[d \frac{r_{\mathrm{H}}}{\tilde{L}}-(d-2) \frac{\tilde{L}}{r_{\mathrm{H}}}\right]
$$

Next we would like to determine the horizon entropy. For Einstein gravity, the Wald formula (2.5) reduces to the expected result $S=\mathcal{A}_{\text {hor }} /\left(4 G_{\mathrm{N}}\right)=\frac{2 \pi}{\ell_{\mathrm{P}}^{d-1}} \mathcal{A}_{\text {hor }}$ and hence, in the present case, eq. (2.7) reduces to

$$
S=\frac{2 \pi}{\ell_{\mathrm{P}}^{d-1}} r_{\mathrm{H}}^{d-1} V_{\Sigma}
$$

Now we would like to use these results to determine the Rényi entropy using eq. (2.6). First we must re-express the temperature (2.15) and the entropy (2.16) as functions of the variable $x=r_{\mathrm{H}} / \tilde{L}$ :

$$
T(x)=\frac{T_{0}}{2}\left[d x-\frac{d-2}{x}\right], \quad S(x)=2 \pi V_{\Sigma} \frac{\tilde{L}^{d-1}}{\ell_{\mathrm{P}}^{d-1}} x^{d-1} .
$$

Next we must determine the lower endpoints $x_{q}$ of the corresponding integration, which corresponds to $T=T_{0} / q$. From eq. (2.17), we see that $x_{q}$ satisfies

$$
0=d x_{q}^{2}-\frac{2}{q} x_{q}-(d-2) .
$$

We can easily find the roots of this quadratic equation and choosing the real and positive root we have

$$
x_{q}=\frac{1}{q d}\left(1+\sqrt{1-2 d q^{2}+d^{2} q^{2}}\right) .
$$

Further we may note that $x_{q} \leq 1$ for $q \geq 1$. Now it straightforward to show that eq. (2.6) yields

$$
S_{q}=\frac{\pi q}{q-1} V_{\Sigma}\left(\frac{\tilde{L}}{\ell_{\mathrm{P}}}\right)^{d-1}\left(2-x_{q}^{d-2}\left(1+x_{q}^{2}\right)\right)
$$




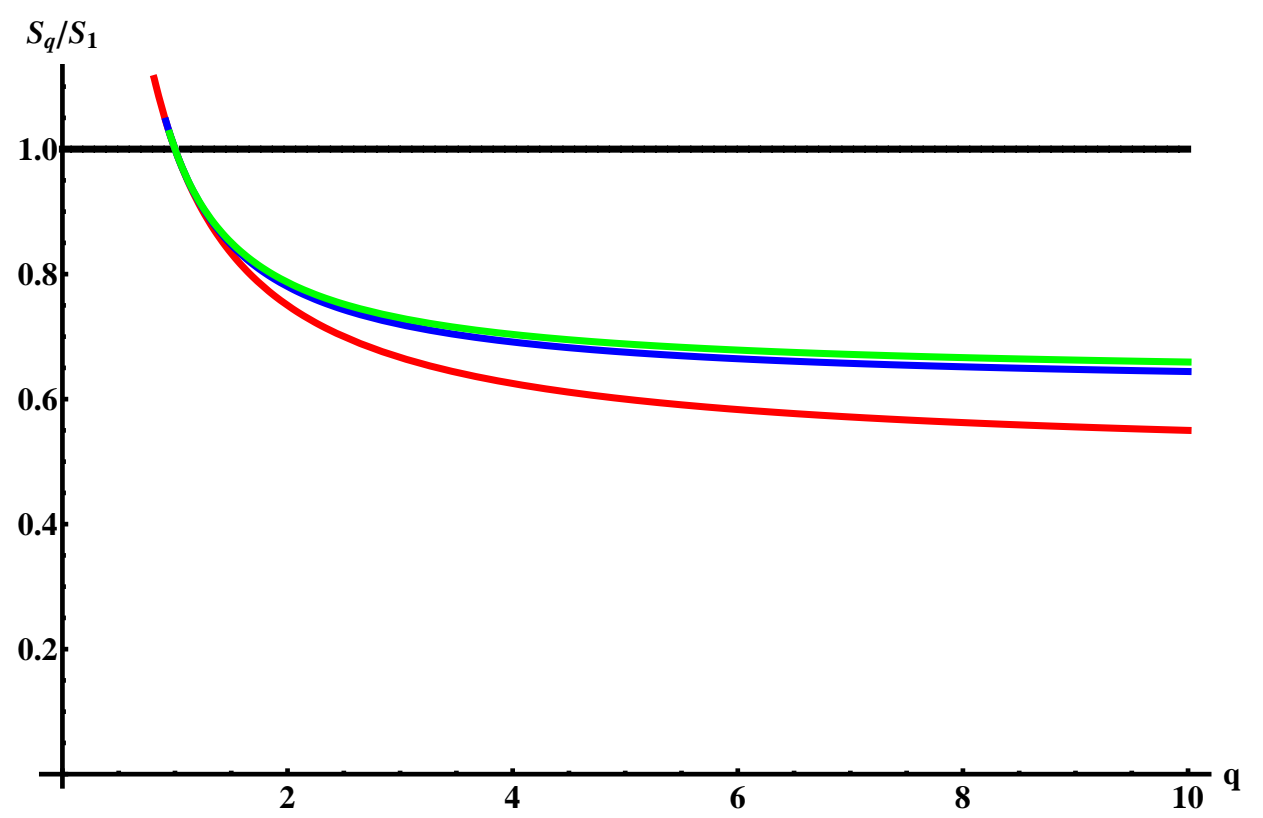

Figure 1: (Colour Online) Ratio of the Rényi entropy to the entanglement entropy, $S_{q} / S_{1}$, as a function of $q$. Starting at the bottom, the three curves correspond to $d=2$ (red), 3 (blue) and 1000 (green). The dashed line with $S_{q} / S_{1}=1$ is simply a guide to the eye. Note that $S_{q} / S_{1}<1$ for $q>1$ and $S_{q} / S_{1}>1$ for $q<1$.

This expression is plotted in Figure 1 for various values of $d$.

As discussed in the introduction, it is interesting to consider the limits $q \rightarrow 0,1$ and $\infty$, which yield

$$
\begin{aligned}
\lim _{q \rightarrow 0} S_{q} & =2 \pi V_{\Sigma}\left(\frac{\tilde{L}}{\ell_{\mathrm{P}}}\right)^{d-1}\left(\frac{2}{d}\right)^{d} \frac{1}{2 q^{d-1}}, \\
\lim _{q \rightarrow 1} S_{q} & =2 \pi V_{\Sigma}\left(\frac{\tilde{L}}{\ell_{\mathrm{P}}}\right)^{d-1}, \\
\lim _{q \rightarrow \infty} S_{q} & =2 \pi V_{\Sigma}\left(\frac{\tilde{L}}{\ell_{\mathrm{P}}}\right)^{d-1}\left(1-\frac{d-1}{d}\left(\frac{d-2}{d}\right)^{\frac{d-2}{2}}\right) .
\end{aligned}
$$

As required, we recover the entanglement entropy in the limit $q \rightarrow 1$, as was calculated previously [9, 21]. This can also be seen by substituting $r_{\mathrm{H}}=\tilde{L}$ in eq. (2.16). We discuss various properties of $S_{0}$ and $S_{\infty}$ later. However, let us note here that because of the factor of $V_{\Sigma}$, both of these expressions (as well as $S_{1}$ ) are divergent in the limit $\delta \rightarrow 0$. 
In this general expression (2.20), we can recognize that the factor $\left(\tilde{L} / \ell_{\mathrm{P}}\right)^{d-1}$ gives a count of the number of degrees of freedom in the dual boundary CFT, e.g., for even $d$, this factor is proportional to the central charge of the boundary theory [27]. ${ }^{5}$ As discussed previously, the factor $V_{\Sigma}$ contains information about the size of the entangling surface and the short-distance cut-off, as given in eqs. (2.10) and (2.11). The remaining factors implicitly give some function of $q$ (and $d$ ) through eq. (2.19). Hence the qualitative form of eq. (2.20) is

$$
S_{q}=C \times V(R / \delta) \times f(q)
$$

where $C$ denotes some central charge in the boundary CFT. Hence this expression, which applies for any $d \geq 2$, has a form similar to the Rényi entropy of any twodimensional CFT given in eq. (1.5). We will see, however, that for holographic CFT's in higher dimensions which are dual to a more general gravitational theory than Einstein gravity, the general form of eq. (2.22) no longer applies.

It is a non-trivial check of our result (2.20) to verify that it yields the expected result (1.5) for a two-dimensional CFT when $d=2$. In this case, eq. 2.18 simplifies to yield $x_{q}=1 / q$ and then eq. (2.20) becomes

$$
S_{q}(d=2)=\frac{\pi \tilde{L}}{\ell_{\mathrm{P}}}\left(1+\frac{1}{q}\right) V_{\Sigma} .
$$

Now we make some observations: first, the central charge of the boundary CFT is given by $c=12 \pi \tilde{L} / \ell_{\mathrm{P}}$, as is well known [27]. Second, care must be taken in evaluating the regulated volume $V_{\Sigma}$. For $d=2$, there are no power law divergences as in eq. (2.10), rather the leading contribution coincides with the universal term in eq. (2.11), i.e., one finds the logarithmic divergence $V_{\Sigma}(d=2)=2 \log (2 R / \delta)+\cdots$. Finally, for $d=2$, the 'spherical' entangling surface consists of two points separated by a distance $2 R$. In other words, we are calculating the Rényi entropy for an interval of length $\ell=2 R$. Combining these observations, eq. (2.23) becomes

$$
S_{q}(d=2)=\frac{c}{6}\left(1+\frac{1}{q}\right) \log \frac{\ell}{\delta},
$$

which precisely matches the required result (2.20) for two dimensions.

\subsection{Gauss-Bonnet gravity and $d \geq 4$}

In this section, we examine holographic Rényi entropy for higher curvature bulk theories by considering Gauss-Bonnet (GB) gravity [18. The gravitational action in $(d+1)$

\footnotetext{
${ }^{5}$ Recall that all of the central charges are proportional to this same factor for any holographic CFT dual to Einstein gravity 27.
} 
dimensions can be written:

$$
I=\frac{1}{2 \ell_{\mathrm{P}}^{d-1}} \int d^{d+1} x \sqrt{-g}\left[\frac{d(d-1)}{L^{2}}+R+\frac{\lambda L^{2}}{(d-2)(d-3)} \mathcal{X}_{4}\right],
$$

where

$$
\mathcal{X}_{4}=R_{a b c d} R^{a b c d}-4 R_{a b} R^{a b}+R^{2}
$$

Of course, because this interaction is proportional to the four-dimensional Euler density, $\mathcal{X}_{4}$ only contributes to the equations of motion for $d \geq 4$ [28]. These higher-dimensional theories (2.25) have a variety of interesting features but foremost amongst these is the fact that the equations of motion are only second order in derivatives [18, which is related to the topological origin of the $\mathcal{X}_{4}$ interaction. Relevant for the present analysis, asymptotically AdS black hole solutions with hyperbolic horizons were found for GB gravity in [29]. We might also mention that [8, 30] provided a generalization of the standard prescription for holographic entanglement entropy (1.1) to these higher curvature theories. However, we will not directly use the results of these studies here.

Recently, there has been renewed interest in this theory (2.25) in the context of the AdS/CFT correspondence - for example, see [31]. One interesting feature of the dual boundary theory is that the different central charges have distinct values because of the curvature-squared interaction [32]. For example, in four dimensions, one has [20]

$$
c=\pi^{2} \frac{\tilde{L}^{3}}{\ell_{\mathrm{P}}^{3}}\left(1-2 \lambda f_{\infty}\right), \quad a=\pi^{2} \frac{\tilde{L}^{3}}{\ell_{\mathrm{P}}^{3}}\left(1-6 \lambda f_{\infty}\right) .
$$

As before, we are using $\tilde{L}$ to denote the curvature scale of the AdS vacuum while $f_{\infty}=L^{2} / \tilde{L}^{2}$. The precise definition of $f_{\infty}$ for GB gravity is given below in eq. (2.35). To facilitate our discussion with arbitrary $d \geq 4$, we would like to define two central charges that appear any CFT for any $d$ - including odd $d$ and hence the trace anomaly is not a useful definition of the central charges. One simple charge is that controlling the leading singularity of the two-point function of the stress tensor. For GB gravity, one finds $33^{6}$

$$
\widetilde{C}_{T}=\frac{\pi^{d / 2}}{\Gamma(d / 2)}\left(\frac{\tilde{L}}{\ell_{\mathrm{P}}}\right)^{d-1}\left[1-2 \lambda f_{\infty}\right]
$$

One can think of this central charge as playing the role of the four-dimensional $c$ in higher dimensions. In fact, with the present normalization, we have $\left.\widetilde{C}_{T}\right|_{d=4}=c$. As

\footnotetext{
${ }^{6}$ Note that the present normalization was chosen so that $\widetilde{C}_{T}=a_{d}^{*}$ in the limit $\lambda \rightarrow 0$. This choice is slightly different from that in 33 , i.e., $C_{T}=\frac{d+1}{d-1} \frac{\Gamma(d+1)}{\pi^{d}} \widetilde{C}_{T}$.
} 
the second central charge, we use that identified in [21] as satisfying an interesting holographic c-theorem:

$$
a_{d}^{*}=\frac{\pi^{d / 2}}{\Gamma(d / 2)}\left(\frac{\tilde{L}}{\ell_{\mathrm{P}}}\right)^{d-1}\left[1-2 \frac{d-1}{d-3} \lambda f_{\infty}\right]
$$

This central charge can be determined in the CFT from the entanglement entropy across a spherical entangling surface [21]. Further it was shown [21] that, in even dimensions, $a_{d}^{*}$ is the central charge appearing in the A-type trace anomaly [34]. For example, comparing with eq. (2.27), we see $\left.a_{d}^{*}\right|_{d=4}=a$. In odd dimensions, this central charge can also be identified with the (renormalized) partition function of the boundary theory evaluated on $S^{d}$ [9]. With this holographic dictionary, eqs. (2.28) and (2.29), we can interpret the results of our gravitational calculations in terms of quantities defined in the dual boundary theory.

Let us now follow the analogous steps as in the previous section to calculate Rényi entropy with GB gravity. First we must determine the function $f(r)$ appearing in the metric (2.1). For GB gravity, the equations of motion lead to the following simple quadratic equation [19]:

$$
f(r)-\lambda f(r)^{2}=1-\frac{\omega^{d}}{r^{d}}
$$

where $\omega^{d}$ is again an integration constant. Combining the above expression with eq. (2.3), we can parameterize this constant in terms of the position of the horizon with

$$
\omega^{d}=r_{\mathrm{H}}^{d}-L^{2} r_{\mathrm{H}}^{d-2}+\lambda L^{4} r_{\mathrm{H}}^{d-4} .
$$

It is straightforward to determine the roots of eq. (2.30) and we find

$$
f(r)=\frac{1}{2 \lambda}\left[1-\sqrt{1-4 \lambda\left(1-\frac{\omega^{d}}{r^{d}}\right)}\right] .
$$

We should note that, in general, eq. (2.30) has two roots and we are only considering that which yields the solution (2.13) of the Einstein theory in the limit $\lambda \rightarrow 0$. One finds that graviton fluctuations about the solution given by the other root are ghosts [35, 19] and hence the boundary theory would not be unitary. Demanding a reasonable holographic framework (i.e., demanding that the dual theory is causal or does not produce negative energy excitations) produces further constraints. In particular, the gravitational coupling must lie within [31, 33]

$$
-\frac{(3 d+2)(d-2)}{4(d+2)^{2}} \leq \lambda \leq \frac{(d-2)(d-3)\left(d^{2}-d+6\right)}{4\left(d^{2}-3 d+6\right)^{2}}
$$


In terms of the central charges, these bounds are conveniently written as

$$
\frac{d(d-3)}{d(d-2)-2} \leq \frac{\widetilde{C}_{T}}{a_{d}^{*}} \leq \frac{d}{2}
$$

We also observe here that $f_{\infty}=(1-\sqrt{1-4 \lambda}) /(2 \lambda)$ and hence $\tilde{L}=L / \sqrt{f_{\infty}} \neq L$ for nonvanishing $\lambda$, i.e., the AdS curvature scale is distinct from the scale $L$ appearing in the action (2.25). Further, we note that from eq. (2.30), the constant $f_{\infty}$ satisfies

$$
1-f_{\infty}+\lambda f_{\infty}^{2}=0
$$

which may be used to simplify various expressions in the following.

Applying eq. (2.4) to the above solution (2.32) we find that the temperature can be expressed as

$$
T=\frac{1}{2 \pi R} \frac{1}{x}\left(1+\frac{d}{2 f_{\infty}} \frac{x^{4}-f_{\infty} x^{2}+\lambda f_{\infty}^{2}}{x^{2}-2 \lambda f_{\infty}}\right),
$$

where, as in the previous section, we used $x=r_{\mathrm{H}} / \tilde{L}$. Next the horizon entropy is calculated to $\mathrm{be}^{7}$

$$
\begin{aligned}
S & =\frac{2 \pi}{\ell_{\mathrm{P}}^{d-1}} \int d^{d-1} x \sqrt{h}\left[1+\frac{2 \lambda L^{2}}{(d-3)(d-2)} \mathcal{R}\right] \\
& =2 \pi \frac{\tilde{L}^{d-1}}{\ell_{\mathrm{P}}^{d-1}} V_{\Sigma} x^{d-1}\left(1-2 \lambda f_{\infty} \frac{d-1}{d-3} \frac{1}{x^{2}}\right) .
\end{aligned}
$$

Then applying eq. (2.6) we arrive at the following expression for the Rényi entropy

$$
\begin{aligned}
S_{q}=\frac{\pi q}{q-1} V_{\Sigma}\left(\frac{\tilde{L}}{\ell_{\mathrm{P}}}\right)^{d-1}[ & \frac{1}{f_{\infty}}\left(1-x_{q}^{d}\right)-\frac{3}{d-3}\left(1-x_{q}^{d-2}\right)-\frac{d-1}{d-3} \lambda f_{\infty}\left(1-x_{q}^{d-4}\right) \\
& \left.+\frac{d}{d-3}(1-4 \lambda)\left(\frac{1}{1-2 \lambda f_{\infty}}-\frac{x_{q}^{d}}{x_{q}^{2}-2 \lambda f_{\infty}}\right)\right]
\end{aligned}
$$

where again $x_{q}$ corresponds to the value of $x$ when the horizon temperature is $T=T_{0} / q$. Using eq. (2.36), we find that the latter is the root of a quartic equation:

$$
0=\frac{d}{f_{\infty}} x_{q}^{4}-\frac{2}{q} x^{3}-(d-2) x^{2}+\frac{4 \lambda f_{\infty}}{q} x+(d-4) \lambda f_{\infty} .
$$

As a check of these results, one may verify that in the limit $\lambda \rightarrow 0$, they reduce to the correct expressions for Einstein gravity in eqs. (2.18) and (2.20).

${ }^{7}$ Note that the expression in the first line of eq. (2.37) is not precisely the same as the Wald entropy (2.5) but both expression agree when evaluated on a Killing horizon [36, 8]. 
Since these general expressions are quite complicated, in considering the results in more detail, we focus on $d=4$. In this case, we may use eq. (2.27) to express the result (2.38) in terms of the central charges of the boundary CFT:

$$
S_{q}=\frac{q}{q-1} \frac{V_{\Sigma}}{4 \pi}\left(1-x_{q}^{2}\right)\left[(5 c-a) x_{q}^{2}-(13 c-5 a)+16 c \frac{2 c x_{q}^{2}-(c-a)}{(3 c-a) x_{q}^{2}-(c-a)}\right]
$$

and further eq. (2.39) reduces to the following cubic equation:

$$
0=x_{q}^{3}-\frac{3 c-a}{5 c-a}\left(\frac{x_{q}^{2}}{q}+x_{q}\right)+\frac{1}{q} \frac{c-a}{5 c-a} .
$$

This four-dimensional result is still quite complicated. The Rényi entropy for these theories no longer has the simple qualitative form (2.22) that was found for the holographic CFT's dual to Einstein gravity and held for any two-dimensional CFT. Here we would replace eq. (2.22) with $S_{q}=a \times V(R / \delta) \times f(q, c / a)$. In particular, the Rényi entropy depends on both of the two distinct central charges in the boundary theory and the dependence on these central charges can not be factored from the dependence on the index $q$.

Considering the limits $q \rightarrow 0,1$ and $\infty$ in eq. (2.40), we find ${ }^{8}$

$$
\begin{aligned}
& \lim _{q \rightarrow 0} S_{q}=a \frac{2 V_{\Sigma}}{\pi} \frac{1}{8 q^{3}} \frac{(3(c / a)-1)^{4}}{(5(c / a)-1)^{3}}, \\
& \lim _{q \rightarrow 1} S_{q}=a \frac{2 V_{\Sigma}}{\pi}, \\
& \lim _{q \rightarrow \infty} S_{q}=a \frac{2 V_{\Sigma}}{\pi}\left[1-\frac{3}{2} \frac{(c / a)^{2}}{5(c / a)-1}\right] .
\end{aligned}
$$

As required, in the limit $q \rightarrow 1$, we recover the entanglement entropy as was calculated previously [9, 21]. In this case, there is a considerable simplification and in particular this result only depends on the central charge $a$. Of course, it is a general result for any $d=4 \mathrm{CFT}$ that the coefficient of the universal term in $V_{\Sigma}$, given in eq. (2.11), is proportional to $a$ (and is independent of $c$ ) with a spherical entangling surface, as considered in [37]. However, no such simplification arises in either of the other limits with $S_{0}$ and $S_{\infty}$ depending on both $a$ and $c$.

As the Rényi entropy in eq. (2.40) is so complicated, it is useful to analyze the result numerically. For this purpose, we consider the ratio of $S_{q} / S_{1}$, i.e., the ratio of the $q$ 'th Rényi entropy to the entanglement entropy. In dividing by $S_{1}$, the entanglement

\footnotetext{
${ }^{8}$ It may be useful to note the corresponding limits for the physical root of eq. (2.41): $\lim _{q \rightarrow 0} x_{q}=$ $f_{\infty} /(2 q), \lim _{q \rightarrow 1} x_{q}=1$ and $\lim _{q \rightarrow \infty} x_{q}=\sqrt{f_{\infty} / 2}$.
} 


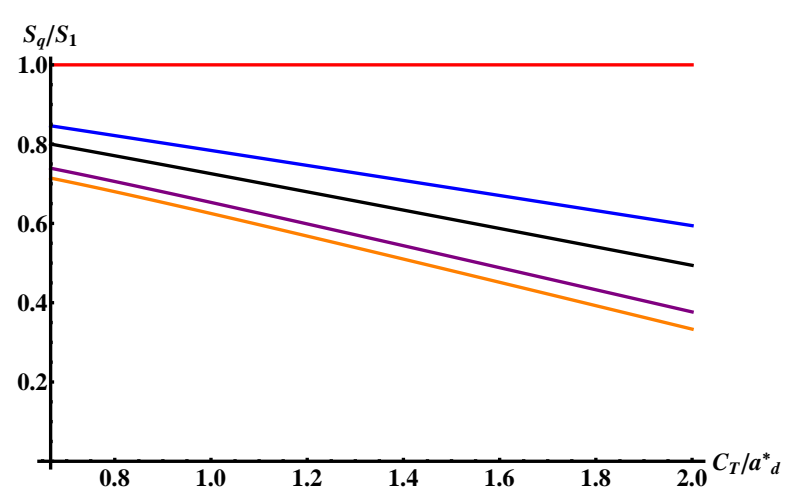

(a)

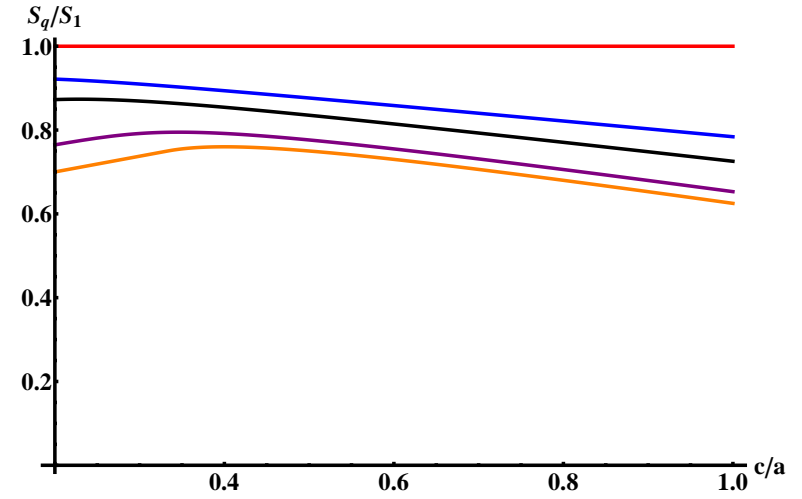

(b)

Figure 2: Rényi entropy (2.40) divided by the entanglement entropy, $S_{q} / S_{1}$ plotted as a function of $c / a$ for various values of $q=\{1,2,3,10,100\}$ with $d=4$. The corresponding curves run from top to bottom with $q=1$ at the top. In panel (a), the plot runs over the physical range: $2 / 3 \leq c / a \leq 2$. Despite the complicated form of eqs. (2.40) and (2.41), these curves are all essentially linear. In panel (b), we see this nearly linear behaviour breaks down for smaller values of $c / a$.

entropy, we remove the dependence on the regulator volume $V_{\Sigma}$ as well as the overall factor of the central charge $a$, and we are left with a function of $q$ and $c / a$. Figure 2 shows a plot of $S_{q} / S_{1}$ as a function of $c / a$ for various values of $q$. Recall that physical constraints on the holographic framework impose constraints on the allowed values of the gravitational coupling $\lambda$, as given in eq. (2.33), or alternatively on the ration of the two central charges, as given in eq. (2.34). For $d=4$, the latter become [38, 31]:

$$
\frac{2}{3} \leq \frac{c}{a} \leq 2
$$

Hence in figure 2(a), we only plot $S_{q} / S_{1}$ over this physical range. Remarkably, the figure shows that in this range, the resulting curves are essentially linear for any value of $q$. Further $S_{q} / S_{1}$ is a monotonically decreasing function of $c / a$, i.e.,

$$
\left.\frac{S_{q}}{S_{1}}\right|_{c_{1} / a_{1}}>\left.\frac{S_{q}}{S_{1}}\right|_{c_{2} / a_{2}} \quad \text { for } \frac{c_{1}}{a_{1}}<\frac{c_{2}}{a_{2}} .
$$

Some insight into this apparent linearity comes from considering the $q \rightarrow \infty$ limit. 
Using the expressions in eq. (2.42), the ratio of interest can be written as

$$
\begin{aligned}
\frac{S_{\infty}}{S_{1}} & =1-\frac{3}{10} \frac{c}{a} \frac{1}{1-\frac{1}{5}\left(\frac{c}{a}\right)^{-1}} \\
& \simeq-\frac{3}{10} \frac{c}{a}+\frac{47}{50}-\frac{3}{250} \frac{a}{c}-\cdots .
\end{aligned}
$$

In the physical regime, the term $\frac{1}{5}\left(\frac{c}{a}\right)^{-1}$ varies between 0.1 and 0.3 and hence the corresponding expression multiplying $c / a$ in the first line above is approximately constant. In fact, the slope varies by slightly less than $10 \%$ over the physical region (2.43). The suppression of the higher order terms in the expansion in the second line above essentially arises because of the extra factor of $1 / 5$ and so appears to be largely a numerical accident. Using eqs. (2.40) and (2.41), a similar expansion can be made for $S_{q} / S_{1}$ with general $q$ but we do not present the details here. These are essentially expansions for large $c / a$ and, as evident from figure 2(a), numerical factors again strongly suppress the higher order terms even though the ratio of central charges is not particularly large in the physical regime (2.43).

It is straightforward to demonstrate that the Rényi entropy could not have been a linear function of the central charges. The latter must obey the inequality $\partial_{q} S_{q} \leq 0$ [11 - see also appendix A - and hence we should find $S_{q} / S_{1}<1$ for all $q>1$ and any $c / a$. Hence, $S_{q} / S_{1}$ is bounded from above and therefore cannot be linear in $c / a$. In figure 2(b), we plot this ratio for smaller values of $c / a$ outside the physical regime. In this plot, the nonlinearity becomes evident and it can be seen that the curves for $q=10$ and 100 reach a maximum value. From eq. (2.45), one readily finds that the maximum in $S_{\infty} / S_{1}$ occurs at $c / a=2 / 5$. However, we note that this interesting structure arises in a regime where the holographic model has unphysical properties and so we do not wish to put too much weight on the appearance of these maxima. We further note that $S_{\infty} / S_{1}$ becomes negative for $c / a \lesssim 0.214$ or $c / a \gtrsim 3.120$, which is clearly unphysical.

Above we have focused the discussion on $d=4$ for simplicity. It is straightforward to carry out a similar analysis for any value of $d$ - however, recall we are assuming $d \geq 4$ with this holographic model using GB gravity in the bulk - and the results are qualitatively similar to those found above. In particular, one would begin using eqs. (2.28) and (2.29) to translate the Rényi entropy (2.38), as well as eq. (2.39), to expressions in terms of the effective central charges, $\widetilde{C}_{T}$ and $a_{d}^{*}$, in the dual boundary 

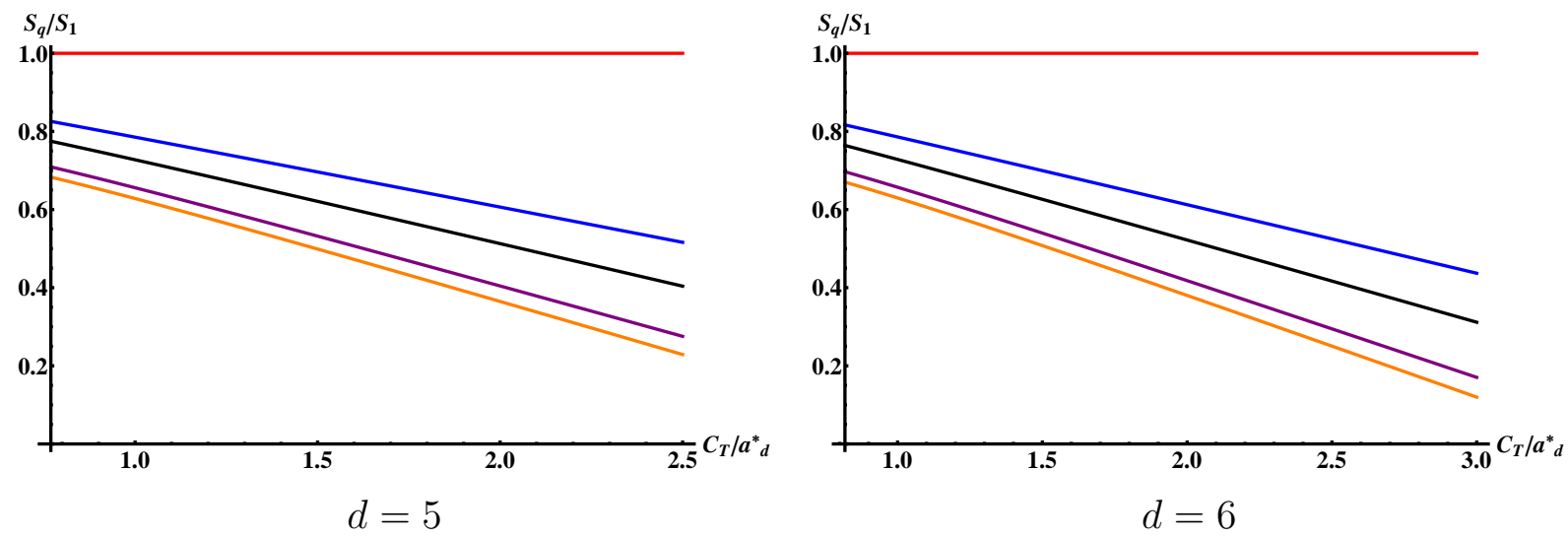

Figure 3: $S_{q} / S_{1}$ plotted as a function of $\widetilde{C}_{T} / a_{d}^{*}$ for $d=5,6$ and for $q=\{1,2,3,10,100\}$. The corresponding curves run from top to bottom with $q=1$ at the top. Despite the complicated form of eqs. (2.38) and (2.39), these curves are all essentially linear in the physical regime of $\widetilde{C}_{T} / a_{d}^{*}$, given in eq. (2.34).

theory. In particular, one may make this translation using

$$
\begin{aligned}
\left(\frac{\tilde{L}}{\ell_{\mathrm{P}}}\right)^{d-1} & =\frac{\Gamma(d / 2)}{2 \pi^{d / 2}} a_{d}^{*}\left((d-1)\left(\widetilde{C}_{T} / a_{d}^{*}\right)-(d-3)\right), \\
\lambda f_{\infty} & =\frac{d-3}{2} \frac{\left(\widetilde{C}_{T} / a_{d}^{*}\right)-1}{(d-1)\left(\widetilde{C}_{T} / a_{d}^{*}\right)-(d-3)},
\end{aligned}
$$

from eqs. (2.28) and (2.29), as well as $1 / f_{\infty}=1-\lambda f_{\infty}$ from eq. (2.35). One then finds expressions which are equally complicated as eqs. (2.40) and (2.41) were for $d=4$. However, one may again plot $S_{q} / S_{1}$ versus $\widetilde{C}_{T} / a_{d}^{*}$ and in the physical regime corresponding to eq. (2.34), the resulting curve is essentially linear. Figure 3 illustrates this behaviour for $d=5$ and 6 .

\subsection{Quasi-topological gravity and $d=4$}

It is well known that, for any even $d$, the universal contribution to the entanglement entropy of a CFT has a logarithmic dependence on the UV cut-off with a coefficient given by some linear combination of the central charges appearing in the trace anomaly [5, 21, 37, 8]. This result applies for any smooth entangling surface and the precise linear combination of central charges depends of the geometries of both the entangling surface and the background in which it is embedded. In the previous section, by extending the bulk theory to GB gravity, we were able to distinguish various (effective) central charges in the boundary theory, as given in eqs. (2.28) and (2.29). However, the subsequent 
holographic calculations demonstrated that the Rényi entropy of the boundary CFT's has a very complicated dependence on these two charges - a property that holds for both even and odd $d \geq 4$. In this section, we will demonstrate that this complexity extends beyond the central charges. In particular, we will focus on the case of even dimensions and show that, in general, the Rényi entropy for a spherical entangling surface depends on CFT parameters beyond the central charges appearing in the trace anomaly.

In the following, we will study a $d=4$ holographic model with quasi-topological gravity as the bulk theory [19, 20]. ${ }^{9}$ The action is given by

$$
I=\frac{1}{2 \ell_{\mathrm{P}}^{3}} \int \mathrm{d}^{5} x \sqrt{-g}\left[\frac{12}{L^{2}}+R+\frac{\lambda L^{2}}{2} \mathcal{X}_{4}+\frac{7 \mu L^{4}}{4} \mathcal{Z}_{5}\right]
$$

where $\mathcal{X}_{4}$ is the same curvature-squared term (2.26) appearing in GB gravity and $\mathcal{Z}_{5}$ is a new third-order interaction originally constructed in [19, 39]:

$$
\begin{aligned}
\mathcal{Z}_{5}=R_{a b}^{c d} R_{c d}^{e f} R_{e f}^{a b}+\frac{1}{56}\left(21 R_{a b c d} R^{a b c d} R-72 R_{a b c d} R^{a b c}{ }_{e} R^{d e}\right. \\
\left.+120 R_{a b c d} R^{a c} R^{b d}+144 R_{a}{ }^{b} R_{b}{ }^{c} R_{c}{ }^{a}-132 R_{a}^{b} R_{b}{ }^{a} R+15 R^{3}\right) .
\end{aligned}
$$

This action (2.47) was constructed to provide a holographic model where the threepoint function of the stress tensor in the boundary CFT had the most general possible form [19]. In any CFT with $d \geq 4$, this three-point function is fixed by conformal invariance up to three independent constants. For $d=4$, we may characterize these three constants as the two central charges, $a$ and $c$, and a third parameter $t_{4}$ which naturally arises in describing certain scattering experiments. ${ }^{10}$ Given the action (2.47), the appropriate AdS/CFT dictionary reads [20]:

$$
\begin{aligned}
c & =\pi^{2} \frac{\tilde{L}^{3}}{\ell_{\mathrm{P}}^{3}}\left(1-2 \lambda f_{\infty}-3 \mu f_{\infty}^{2}\right), \\
a & =\pi^{2} \frac{\tilde{L}^{3}}{\ell_{\mathrm{P}}^{3}}\left(1-6 \lambda f_{\infty}+9 \mu f_{\infty}^{2}\right), \\
t_{4} & =\frac{2 \mu f_{\infty}^{2}}{1-2 f_{\infty} \lambda-3 \mu f_{\infty}^{2}} .
\end{aligned}
$$

\footnotetext{
${ }^{9}$ It is straightforward to extend the following analysis to higher values of $d$ but the qualitative behaviour of the Rényi entropy does not change. In particular, the final result explicitly depends on the new parameter $t_{4}$, as well as the two central charges.

${ }^{10}$ We refer the interested reader to [38, 20 for further details. We also note that to simplify various expressions in the following, we have changed the normalization of this parameter with respect to these previous references, i.e., $\left(t_{4}\right)_{\text {previous }}=1890\left(t_{4}\right)_{\text {present }}$.
} 
As usual, $\tilde{L}$ denotes the AdS curvature scale while $f_{\infty}=L^{2} / \tilde{L}^{2}$. The precise definition for $f_{\infty}$ in quasi-topological gravity is given below in eq. (2.52). Note that if $\mu$ is set to zero, we recover the expressions in eq. (2.27) for the central charges in GB gravity and $t_{4}$ vanishes.

By design, the metric function $f(r)$ in eq. (2.1) is still determined as the root of a simple polynomial [19]

$$
f(r)-\lambda f(r)^{2}-\mu f(r)^{3}=1-\frac{\omega^{4}}{r^{4}}
$$

The integration constant $\omega^{4}$ can again be written in terms of the position of the horizon

$$
\omega^{4}=r_{\mathrm{H}}^{4}-L^{2} r_{\mathrm{H}}^{2}+\lambda L^{4}+\mu L^{6} / r_{\mathrm{H}}^{2}
$$

As noted previously, the asymptotic value of $f(r)$ fixes the AdS scale with $\tilde{L}=L / \sqrt{f_{\infty}}$. In the present case, in the limit $r \rightarrow \infty$, eq. (2.50) reduces to the following cubic equation for $f_{\infty}$ :

$$
1-f_{\infty}+\lambda f_{\infty}^{2}+\mu f_{\infty}^{3}=0
$$

Demanding that the boundary theory does not produce negative energy excitations constrains the two higher curvature couplings, $\lambda$ and $\mu$, to satisfy [20]:

$$
\begin{aligned}
& 0 \leq 1-10 \lambda f_{\infty}+189 \mu f_{\infty}^{2}, \\
& 0 \leq 1+2 \lambda f_{\infty}-855 \mu f_{\infty}^{2}, \\
& 0 \leq 1+6 \lambda f_{\infty}+1317 \mu f_{\infty}^{2} .
\end{aligned}
$$

Within this physical domain, the couplings remain relatively small. Further, in this regime, eq. (2.52) will have three roots but implicitly we only consider the smallest positive root, as it is the only one to yield a physically reasonable holographic model without ghosts 35, 19].

The temperature of these black hole solutions can be calculated using eq. (2.4) and is given by

$$
T=\frac{1}{2 \pi R} \frac{1}{x}\left(1+\frac{2}{f_{\infty}} \frac{x^{6}-f_{\infty} x^{4}+\lambda f_{\infty}^{2} x^{2}+\mu f_{\infty}^{3}}{x^{4}-2 \lambda f_{\infty} x^{2}-3 \mu f_{\infty}^{2}}\right),
$$

where as before $x \equiv r_{\mathrm{H}} / \tilde{L}$. Calculating the entropy using the Wald formula (2.7) yields [19:

$$
S=2 \pi \frac{\tilde{L}^{3}}{\ell_{\mathrm{P}}^{3}} V_{\Sigma} x^{3}\left(1-6 \lambda f_{\infty} \frac{1}{x^{2}}+9 \mu f_{\infty}^{2} \frac{1}{x^{4}}\right) .
$$


Combining the above results with eq. (2.6), the Rényi entropy for a spherical entangling surface in the $d=4$ boundary CFT is

$$
\begin{aligned}
S_{q}= & \frac{\pi q}{q-1} V_{\Sigma} \frac{\tilde{L}^{3}}{\ell_{\mathrm{P}}^{3}}\left(1-x_{q}^{2}\right)\left\{\frac{1+x_{q}^{2}}{f_{\infty}}+(1-16 \lambda)-\frac{3 \mu f_{\infty}^{2}}{x_{q}^{2}}-16 f_{\infty}^{2}\right. \\
& \left.\times\left[\frac{\left((1-4 \lambda) \lambda^{2}+3 \mu \lambda-2 \mu\right)\left(x_{q}^{2}+3 \mu f_{\infty}^{2}\right)+3 \mu f_{\infty}((1-2 \lambda) \lambda+3 \mu)\left(1+x_{q}^{2}-2 \lambda f_{\infty}\right)}{\left(1-2 \lambda f_{\infty}-3 \mu f_{\infty}^{2}\right)\left(x_{q}^{4}-2 \lambda f_{\infty} x_{q}^{2}-3 \mu f_{\infty}^{2}\right)}\right]\right\} .
\end{aligned}
$$

As before, $x_{q}$ is defined by the relation $T=T_{0} / q$. In this case, eq. (2.54) yields the following sixth order polynomial to solve for $x_{q}$ :

$$
0=\frac{2}{f_{\infty}} x_{q}^{6}-\frac{1}{q} x_{q}^{5}-x_{q}^{4}+\frac{2 \lambda f_{\infty}}{q} x_{q}^{3}+\frac{3 \mu f_{\infty}^{2}}{q} x_{q}-\mu f_{\infty}^{2}
$$

Although it is not immediately obvious, the $\mu \rightarrow 0$ limit correctly reproduces the corresponding expressions for GB gravity in eqs. (2.40) and (2.39), respectively.

The above expressions are readily rewritten in terms of the CFT parameters using eq. (2.49), which yields

$$
\begin{aligned}
\frac{\tilde{L}^{3}}{\ell_{\mathrm{P}}^{3}} & =\frac{a}{2 \pi^{2}}\left(3 \frac{c}{a}\left(1+3 t_{4}\right)-1\right) \\
\lambda f_{\infty} & =\frac{1}{2} \frac{\frac{c}{a}\left(1+3 t_{4}\right)-1}{3 \frac{c}{a}\left(1+3 t_{4}\right)-1}, \\
\mu f_{\infty}^{2} & =\frac{\frac{c}{a} t_{4}}{3 \frac{c}{a}\left(1+3 t_{4}\right)-1},
\end{aligned}
$$

as well as eq. (2.52), which yields

$$
\begin{aligned}
\frac{1}{f_{\infty}} & =1-\lambda f_{\infty}-\mu f_{\infty}^{2} \\
& =\frac{1}{2} \frac{5 \frac{c}{a}\left(1-2 t_{4}\right)-1}{3 \frac{c}{a}\left(1+3 t_{4}\right)-1} .
\end{aligned}
$$

Also note that the constraints in eq. (2.53) become

$$
\begin{aligned}
& 2 \geq \frac{c}{a}\left(1-84 t_{4}\right), \\
& \frac{1}{2} \leq \frac{c}{a}\left(1-210 t_{4}\right) \\
& \frac{2}{3} \leq \frac{c}{a}\left(1+224 t_{4}\right)
\end{aligned}
$$


We will not explicitly display eqs. 2.56) and (2.57) after their conversion to CFT parameters since they are rather cumbersome and not particularly enlightening. Of course, one important feature which they do demonstrate is that the Rényi entropies have a very complicated dependence on these parameters characterizing the boundary CFT. As in the previous section with GB gravity in the bulk, the Rényi entropy no longer has the simple qualitative form (2.22) that appeared for CFT's dual to Einstein gravity. Instead the qualitative form of the results is $S_{q}=a \times V(R / \delta) \times f\left(q, c / a, t_{4}\right)$. In particular then, the Rényi entropy depends on the new parameter $t_{4}$, as well as both of the central charges. Demonstrating this additional dependence of the Rényi entropy on parameters beyond the central charges in an even-dimensional CFT was the main goal of the present section.

For a fixed value of $t_{4}$, the Rényi entropy here has the same qualitative shape as with GB gravity in the previous section (for which $t_{4}=0$ ). In particular, within the physical regime, $S_{q} / S_{1}$ is nearly linear with a negative slope, similar to the dependence shown in figure 8 . Figure 4 illustrates the new dependence on $t_{4}$. In figure $4(a)$, we see that within the physically allowed regime fixed by eq. (2.60), the dependence of $S_{q} / S_{1}$ on $t_{4}$ (at fixed $c / a$ ) is nearly linear, but in fact the slope is very small. Figure $4(\mathrm{~b})$ shows that the dependence on $t_{4}$ and $c / a$ only becomes significant well outside of the physical regime.

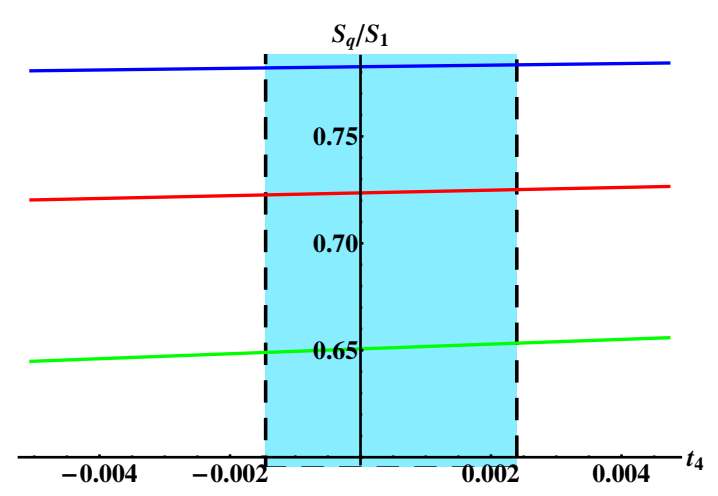

(a)

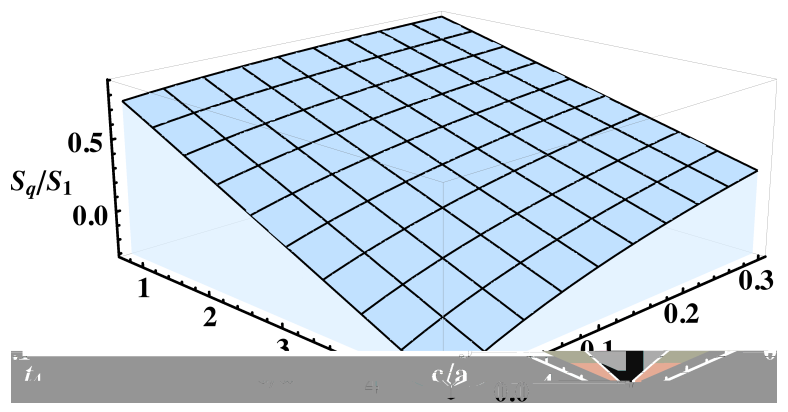

(b)

Figure 4: (Colour Online) $S_{q} / S_{1}$ as a function of $t_{4}$ with fixed $c / a=1$. Starting from the top, the three curves correspond to $q=2$ (blue), 3 (red) and 10 (green). The shaded blue region between the dashed vertical lines corresponds to the allowed physical regime for $t_{4}$ (with $c / a=1$ ) dictated by eq. (2.60). (b) $S_{q} / S_{1}$ as a function of both $t_{4}$ and $c / a$. Note that in this case, the plot extends beyond the physical regime.

Recall that in the previous section, there was a certain simplification in the limits $q \rightarrow 0,1, \infty$. Considering the $q \rightarrow 1$ limit, i.e., the entanglement entropy, first here, we 
find

$$
S_{\mathrm{EE}}=\lim _{q \rightarrow 1} S_{q}=a \frac{2 V_{\Sigma}}{\pi} .
$$

The fact that, for a spherical entangling surface, the entanglement entropy only depends on the central charge $a$ could be anticipated from the holographic calculations of [9, 21] or from the CFT calculations of [37]. Note that this expression matches that given for $S_{1}$ in eq. (2.42) for GB gravity.

Next turning to the limit $q \rightarrow 0$, we find a certain simplification in the Rényi entropy

$$
\begin{aligned}
\lim _{q \rightarrow 0} S_{q} & =V_{\Sigma} \frac{\tilde{L}^{3}}{\ell_{\mathrm{P}}^{3}} \frac{\pi f_{\infty}^{3}}{16 q^{3}} \\
& =\frac{a V_{\Sigma}}{4 \pi q^{3}} \frac{\left(3 \frac{c}{a}\left(1+3 t_{4}\right)-1\right)^{4}}{\left(5 \frac{c}{a}\left(1+2 t_{4}\right)-1\right)^{3}} .
\end{aligned}
$$

As in the previous section, $x_{q}=f_{\infty} / 2 q$ in this limit. We note that the expression in the first line, which expresses $S_{0}$ in terms of the gravitational parameters, takes the same form here for quasi-topological gravity as it did for GB gravity in the previous section. Therefore there is no dependence on the dimensionless couplings for the higher curvature terms except implicitly through the factor of $\tilde{L}^{3} f_{\infty}^{3}=L^{3} f_{\infty}^{3 / 2}$. However, despite this simplification, this expression still depends on $t_{4}$, as well as the central charges.

Finally, we consider the limit $q \rightarrow \infty$. In this case, there is no particular simplification of the final result and so $S_{\infty}$ again depends on all three parameters, i.e., $t_{4}$ as well as the two central charges, $a$ and $c$. To explicitly exhibit the $t_{4}$ dependence, we first observe that eq. (2.60) constrains the physical values of $t_{4}$ to be rather small see, for example, figure $4(\mathrm{a})$. Hence we construct a Taylor series expansion of $s_{\infty}$ for small $t_{4}$ which yields

$$
\begin{aligned}
\lim _{q \rightarrow \infty} S_{q} \simeq & \frac{2 a V_{\Sigma}}{\pi}\left[1+\frac{3}{2} \frac{\left(\frac{c}{a}\right)^{2}}{1-5 \frac{c}{a}}\right. \\
& \left.+t_{4} \frac{1-17 \frac{c}{a}+98\left(\frac{c}{a}\right)^{2}-194\left(\frac{c}{a}\right)^{3}-17\left(\frac{c}{a}\right)^{4}+215\left(\frac{c}{a}\right)^{5}}{2 \frac{c}{a}\left(1-3 \frac{c}{a}\right)\left(1-5 \frac{c}{a}\right)^{2}}+\mathcal{O}\left(t_{4}^{2}\right)\right] .
\end{aligned}
$$

\section{Twist Operators and Regulator Surfaces}

In the previous section, the Rényi entropy for a spherical entangling surface was computed by mapping the calculation to a thermodynamic one [9]. This approach contrasts 
with 'standard' field theoretic computations, particularly in two dimensions [2, 3], which make use of the replica trick. The replica trick essentially replaces the problem of computing the $q$-th power of the density matrix with that of another density matrix of a different theory that comprises $q$ copies of the original theory. The various copies only talk to each other along the $q$-fold branch-cuts introduced along the region of interest. In two dimensions, the latter is generically a collection of line segments and so this construction can be realized with the insertion of twist operators in the path integral over the replicated theory, which are responsible for opening and closing the branch cuts at the end-points of the various intervals. Let us denote the twist operator at the end-point $\omega_{i}$ as $\sigma_{\eta_{i} q}\left(\omega_{i}\right)$ where $\eta_{i}=+1(-1)$ to open (close) a $q$-fold branch-cut. Then the $q$-th power of the reduced density matrix is written

$$
\operatorname{tr}\left[\rho_{V}^{q}\right]=\frac{\int\left[\prod_{j=1}^{q} \mathrm{D} \phi_{j}\right] \prod_{k} \sigma_{\eta_{k} q}\left(\omega_{k}\right) \exp \left[-\sum_{i=1}^{q} I_{i}[\phi]\right]}{\int\left[\prod_{j=1}^{q} \mathrm{D} \phi_{j}\right] \exp \left[-\sum_{i=1}^{q} I_{i}[\phi]\right]} \equiv \frac{Z_{q}}{Z_{1}^{q}} .
$$

which corresponds to the correlation function of these twist operators. Here $Z_{q}$ denotes the path integral of the replicated theory on the orbifold, and $Z_{1}$ that of the original theory on the original background. In the case of a single interval, the Rényi entropy is given by the correlation function of just two twist operators and the conformal dimension of these operators then completely fixes the form of this correlator.

The idea above of introducing branch-cuts immediately generalizes to higher dimensional theories. Hence applying the replica trick to calculate Rényi entropies in higher dimensions should also be a straightforward extension of the previous discussion. However, in practice the construction and properties of the corresponding twist operators are not well understood for $d>2$ - however, see [26, 40]. Twist operators in a $d$-dimensional theory would be some $(d-2)$-dimensional surface operators. From previous work on various surface operators and their holographic duals 41, it seems that Ryu and Takayanagi's holographic prescription [4] for entanglement entropy is in agreement with this interpretation. For a three-dimensional CFT, for example, the holographic entanglement entropy computation is essentially the same as that of the vacuum expectation value of a spatial 'Wilson-line' operator. As so little is known about these operators, it would of interest to use holography to study their general properties, e.g., the conformal dimension, as this could shed light on the explicit construction of these objects. We return to these considerations in section 4 In the present section, we will begin with a discussion of two-dimensional CFT's and the corresponding holographic description of calculations using twist operators.

\subsection{Twist operators in $d=2$}

Before considering the holographic calculation, let us review the computation the Rényi 
entropy $S_{q}$ in a two-dimensional CFT using the replica trick in more detail. In particular, we calculate $S_{q}$ for a single interval $V$ bounded by two points $\omega=v_{1}$ and $\omega=v_{2}$ in the complex $\omega$-plane. Without loss of generality we can put both $v_{1}$ and $v_{2}$ on the real line, with $v_{2}>v_{1}$. Recall the general definition (1.2) of the entanglement entropy in terms of the reduced density matrix $\rho_{V}$ :

$$
S_{q}=\frac{1}{1-q} \log \operatorname{tr}\left[\rho_{V}^{q}\right] .
$$

As explained above, using the replica trick, one computes instead the path integral of a theory where $q$ copies of all the fields are introduced accompanied by the insertion of appropriate twist operators at the end-points, $v_{1}$ and $v_{2}$. Explicitly, eq. (3.1) reduces to

$$
\operatorname{tr}\left[\rho_{V}^{q}\right]=\frac{Z_{q}}{Z_{1}^{q}}=\left\langle\sigma_{q}\left(v_{1}\right) \sigma_{-q}\left(v_{2}\right)\right\rangle .
$$

The branch points at which the twist operators are inserted are singular points in the universal cover of the $\omega$-plane, suffering an angular excess of $2 \pi(q-1)$. However, in $d=2$, all metrics are conformally flat. Hence the singular metric on the universal cover can be mapped to a flat metric by a conformal transformation. In particular, the transformation [2]

$$
z=\left(\frac{\omega-v_{1}}{\omega-v_{2}}\right)^{\frac{1}{q}}
$$

maps the singular $\omega$-plane to the smooth $z$-plane. From eq. (3.4), we easily see that $\omega=v_{1} \rightarrow z=0$ and $\omega=v_{2} \rightarrow z=\infty$ and hence, this transformation maps the interval between $v_{1}$ and $v_{2}$ in the $w$-plane onto the half-line in the $z$-plane. The power $1 / q$ ensures that the singularity in the universal cover is smoothed out by patching together $q$ copies of $\omega$-planes. In terms of the $z$ coordinate, each $\omega$-plane is a wedge subtending an angle of $2 \pi / q$. The $q$ wedges are sewn together along edges which correspond to the interval between $v_{1}$ and $v_{2}$ on either side of the branch-cut, as illustrated in figure 5 . We also observe that $\omega=\infty$ is mapped to the $q$-th roots of unity in the $z$-plane. The above transformation (3.4) implies that

$$
d z d \bar{z}=\frac{\left(v_{2}-v_{1}\right)^{2}}{q^{2}} \frac{d \omega d \bar{\omega}}{\left|\left(\omega-v_{1}\right)^{(1-1 / q)}\left(\omega-v_{2}\right)^{(1+1 / q)}\right|^{2}} .
$$

This line element explicitly illustrates that the curvature singularities in the $\omega$-plane can be completely absorbed by a conformal factor. Finally, let us note that with $q=1$ the transformation (3.4) is in fact the Euclidean version of the map to the Rindler wedge for $d=2$ discussed in [9]. 


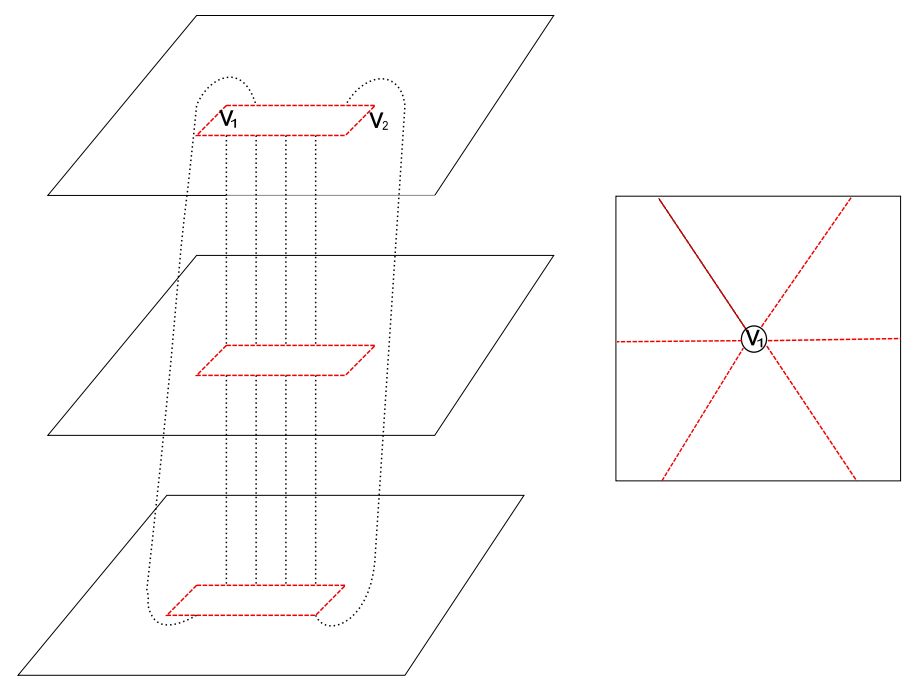

Figure 5: The picture on the left depicts the universal cover of the $\omega$-orbifold. The dashed lines indicate the identifications between planes across the branch cut. The right picture depicts the image of the universal cover in the $z$-plane under the conformal mapping given in eq. (3.4).

Since the $z$-plane is flat and preserves translation invariance, $\left\langle T_{z z}(z)\right\rangle=0$, where $T_{z z}(z)$ is the holomorphic part of the stress tensor of the two-dimensional CFT. Hence, transforming back to the $\omega$-plane, we have ${ }^{11}$

$$
\left\langle T_{\omega \omega}(\omega)\right\rangle=-\frac{c}{24 \pi}\{z, \omega\}=-\frac{c}{48 \pi}\left(1-\frac{1}{q^{2}}\right) \frac{\left(v_{2}-v_{1}\right)^{2}}{\left(\omega-v_{2}\right)^{2}\left(\omega-v_{1}\right)^{2}},
$$

where $c$ is the central charge, and $\{z, \omega\}$ is the Schwarzian derivative. That is,

$$
\{z, \omega\}=\frac{z^{\prime \prime \prime}(\omega)}{z^{\prime}(\omega)}-\frac{3}{2}\left(\frac{z^{\prime \prime}(\omega)}{z^{\prime}(\omega)}\right)^{2} .
$$

\footnotetext{
${ }^{11}$ Note that the present conventions are such that $T_{\mu \nu}=\frac{2}{\sqrt{g}} \frac{\delta I_{E}}{\delta g^{\mu \nu}}$. We refer the interested reader to 21 for a full discussion of our conventions but let us note here that the present conventions disagree with those in [2, 3]. Comparing to these original references, $\left(T_{\mu \nu}\right)_{\text {original }}=-2 \pi\left(T_{\mu \nu}\right)_{\text {present }}$. However, in defining the scaling dimensions below, we write the OPE of (the holomorphic part of) the stress tensor with a primary operator as

$$
T_{\omega \omega}(\omega) \mathcal{O}(z, \bar{z}) \simeq-\frac{\Delta}{2 \pi} \frac{\mathcal{O}(z, \bar{z})}{(\omega-z)^{2}}+\cdots
$$

and hence our result in eq. (3.11) is identical to that presented in [3]. Of course, the total scaling dimension there is given by $h=\Delta+\bar{\Delta}$.
} 
As explained in [3], this expectation value (3.7) is actually for the stress tensor in the presence of the twist operators, i.e.,

$$
\left\langle T_{\omega \omega}(\omega)\right\rangle=\frac{\left\langle T_{\omega \omega}(\omega) \sigma_{q}\left(v_{1}\right) \sigma_{-q}\left(v_{2}\right)\right\rangle}{\left\langle\sigma_{q}\left(v_{1}\right) \sigma_{-q}\left(v_{2}\right)\right\rangle} .
$$

This is however, the result from the insertion of $T_{\omega \omega}$ for a single copy of the field theory, i.e., on one sheet of the universal cover [3]. If we insert $T_{\omega \omega}$ on all $q$ sheets, the right-hand side of eq. (3.7) acquires an extra factor of $q$ to become

$$
\left\langle T_{\omega \omega}(\omega)\right\rangle=-\frac{q c}{48 \pi}\left(1-\frac{1}{q^{2}}\right) \frac{\left(v_{2}-v_{1}\right)^{2}}{\left(\omega-v_{2}\right)^{2}\left(\omega-v_{1}\right)^{2}} .
$$

Keeping in mind the correlator in eq. (3.9), we can easily read off the conformal dimension of the twist operators by considering the limits $\omega \rightarrow v_{2}, v_{1}$ in eq. (3.10) and its complex conjugate. One finds that both $\sigma_{ \pm q}$ are (spinless) primary operators, where holomorphic and anti-holomorphic scaling dimensions, $\Delta_{q}$ and $\bar{\Delta}_{q}$, are both given by the pre-factor in the expression above. Adding these two results, the total scaling dimension of both twist operators is given by

$$
h_{q}=2 \Delta_{q}=\frac{q c}{12}\left(1-\frac{1}{q^{2}}\right)=\frac{c}{12}\left(q-\frac{1}{q}\right) .
$$

Hence we thus have

$$
\operatorname{tr}\left[\rho_{V}^{q}\right]=\left\langle\sigma_{q}\left(v_{1}\right) \sigma_{-q}\left(v_{2}\right)\right\rangle=s_{q}\left|\frac{v_{2}-v_{1}}{\delta}\right|^{-2 h_{q}},
$$

where $\delta$ is the short-distance UV cut-off and $s_{q}$ is some constant. Since $\operatorname{tr}\left[\rho_{V}\right]=1$, we know $s_{1}=1$. Otherwise, it is not determined purely from conformal symmetry and depends on the details of the CFT. However, $s_{q}$ will not effect the universal contribution to the Rényi entropy. Substituting the above expression into eq. (3.2), we reproduce the well known result (1.5) for the Rényi entropy of a single interval for any two-dimensional CFT.

\subsection{Holographic calculation for $d=2$}

Let us now turn to the holographic description of the above calculation with twist operators in $d=2$. In the previous section, we saw that the action of the twist operators was captured by the conformal transformation (3.4) and hence the expectation value of the stress tensor was simply given by the Schwarzian in eq. (3.7). As a result, the conformal dimension of the twist operators in a two-dimensional CFT is a universal 
quantity depending only on the central charge of the theory. We expect that we should be able to recover precisely this dimension (3.11) and the corresponding two-point correlator (3.12) with our holographic calculations.

Related holographic calculations of Rényi entropies based on the replica trick first appeared in [13. However, the approach presented there yields incorrect results because of singularities in the bulk geometry [7]. An alternative approach avoiding such singularities was explored in 42, although there is no natural way there to obtain the finite contribution to the partition function. The discussion there is closely related to our analysis below. In the following, we will demonstrate a bulk calculation that closely parallels that presented in the previous section for the boundary CFT. Unlike the derivation reviewed above, we will directly evaluate the path integral in the presence of the twist operators from the classical gravity action. The extra factor of $q$ acquired from the $q$-sheets in the final answer would emerge naturally from the geometry. In many respects, our bulk calculations closely resemble the CFT calculations found in [43, which considered correlation functions of twist operators in a symmetric orbifold CFT, which are relevant for the microscopic description of certain black holes in string theory.

The standard holographic prescription dictates that we should work with an asymptotically AdS bulk geometry that extremizes the gravity action, and that the AdS boundary is chosen to coincide with the geometry in which the CFT correlator of the twist operators is evaluated. From the discussion above, the boundary should be the universal cover of the $\omega$-plane. One could alternatively work with the $z$ coordinate, in which case these curvature singularities are taken into account by the conformal factor in eq. (3.5). However, in the following, we find it more convenient to work with the $\omega$ coordinate. Nevertheless one should bear in mind that $\omega$-plane only corresponds to a single patch of the universal cover.

At this point, it is most systematic to work in the Fefferman-Graham (FG) gauge 44 - see also 45 - where the asymptotically $\mathrm{AdS}_{3}$ metric reads

$$
d s^{2}=\frac{L^{2} d \rho^{2}}{4 \rho^{2}}+\frac{g_{a b}(\omega, \bar{\omega}, \rho) d x^{a} d x^{b}}{\rho},
$$

with

$$
g_{a b}(\omega, \bar{\omega}, \rho)=g_{(0) a b}+\rho g_{(2) a b}+\rho^{2} g_{(4) a b}+\cdots .
$$

The relevant boundary metric $g_{(0) a b}$ in the present case is simply

$$
d s_{(0)}^{2}=g_{(0) a b} d x^{a} d x^{b}=d \omega d \bar{\omega} .
$$

Despite appearances, we again remind ourselves that this metric (3.15) is not everywhere flat. Rather we are thinking of this as a single patch of the universal cover which 
has curvature singularities at $\omega=v_{2}$ and $\omega=v_{1}$. Again, the above metric is related to the flat metric in the $z$-plane by the conformal factor shown in eq. (3.5). In any event, since the boundary is conformally flat, the bulk solution is still simply pure $\mathrm{AdS}_{3}$ space.

A procedure to generate pure $\mathrm{AdS}_{3}$ solutions for given conformally flat boundary metric defined in the FG gauge was introduced in [46]. We will make use of these techniques in what follows to illustrate some features of the holographic calculation. However, as it turns out, the computation of Rényi entropy does not require the full machinery of [46]. Hence we relegate most of the details to appendix Q, quoting only the salient results here. In particular, following this analysis for a pure $\mathrm{AdS}_{3}$ with an arbitrary boundary metric $g_{(0)}$ in the FG gauge, the on-shell gravity action (C.18) reduces to

$$
I_{\mathrm{tot}}=-\frac{c}{12 \pi} \int d \omega d \bar{\omega} \sqrt{g_{(0)}}\left[L^{2} \frac{R_{(0)}}{4} \ln \rho_{\text {crit }}^{-}+\frac{1}{2} \sqrt{\left|\mathcal{T}_{\omega \omega}\right|^{2}}+\frac{3}{8} L^{2} R_{(0)}\right] .
$$

where $R_{(0)}$ is the Ricci scalar for $g_{(0)}$ and $\rho_{\text {crit }}^{-}$is the upper limit of the radial integral. As explained in the appendix, the latter is determined by where the measure of the bulk metric first reaches zero. Further $\mathcal{T}$ is a tensor appearing in evaluating $g_{(2)}$ and is proportional to the stress tensor of the boundary CFT, as shown in eq. (C.16). Recall that $c=12 \pi L / \ell_{\mathrm{P}}$ is the central charge of (a single copy of) the boundary CFT. Explicit expressions for $\rho_{\text {crit }}^{-}$and $\mathcal{T}_{\omega \omega}$ are given in eqs. (C.23) and (C.15), respectively.

Now, the boundary curvature (i.e., $R_{(0)}$ for the universal cover) and $\mathcal{T}_{\omega \omega}$ are both singular at $\omega=v_{2}$ and $v_{1}$. Hence to evaluate the above action (3.16), we cut-off the integration in the $\omega$-plane with

$$
\left|\omega-v_{2}\right|>\delta \quad \text { and } \quad\left|\omega-v_{1}\right|>\delta
$$

where $\delta$ is again the short-distance cut-off in the boundary theory. That is, we are regulating this integral with the same cut-off that appears, for example, in evaluating $V_{\Sigma}$ in section 2 - see eqs. (2.10) and (2.11). This regulator simplifies the integrand since $R_{(0)}$ vanishes away from the two singular points. Hence upon substituting eq. C.15 for $\mathcal{T}_{\omega \omega}$, the gravity action (3.16) becomes

$$
\begin{aligned}
I_{\mathrm{tot}} & =-\frac{c}{24 \pi} \int d \omega d \bar{\omega} \frac{1}{2}\left(1-\frac{1}{q^{2}}\right) \frac{\left(v_{2}-v_{1}\right)^{2}}{\left|\omega-v_{2}\right|^{2}\left|\omega-v_{1}\right|^{2}} \\
& =-\frac{c}{48 \pi}\left(1-\frac{1}{q^{2}}\right) \int d \omega d \bar{\omega}\left|\partial_{\omega}\left(\ln \left|\frac{\omega-v_{1}}{\omega-v_{2}}\right|^{2}\right)\right|^{2} \\
& =-\frac{c}{96 \pi}\left(1-\frac{1}{q^{2}}\right) \int d \omega d \bar{\omega} \sqrt{g_{(0)}} g_{(0)}^{a b} \partial_{a} \chi \partial_{b} \chi,
\end{aligned}
$$


where we have introduced a convenient auxiliary field

$$
\chi=\ln \left|\frac{\omega-v_{1}}{\omega-v_{2}}\right|^{2} .
$$

Now we integrate by parts in the above integral to find

$$
I_{\text {tot }}=-\frac{c}{96 \pi}\left(1-\frac{1}{q^{2}}\right) \int d \omega d \bar{\omega}\left[\sqrt{g_{(0)}} \nabla_{a}\left(\chi g_{(0)}^{a b} \nabla_{b} \chi\right)-2 \chi \partial_{\omega} \partial_{\bar{\omega}} \chi\right] .
$$

Again since the integrand is evaluated away from the singular points as in eq. (3.17), the second term above vanishes. Now the integral reduces to two boundary terms integrated around the edges of the two cut-off disks (3.17) at $\omega=v_{2}$ and $v_{1}$ in the universal cover. Adopting polar coordinates in the vicinity of the singular points, e.g., $\omega-v_{2} \simeq r e^{i \theta}$, these boundary terms yield

$$
\begin{aligned}
I_{\mathrm{tot}} & =-\frac{c}{96 \pi}\left(1-\frac{1}{q^{2}}\right)\left(\oint_{\omega=v_{2}}+\oint_{\omega=v_{1}}\right) d \theta\left[\delta \times \chi \partial_{r} \chi\right] \\
& =\frac{q c}{6}\left(1-\frac{1}{q^{2}}\right) \ln \left(\frac{v_{2}-v_{1}}{\delta}\right) .
\end{aligned}
$$

Note that the overall factor of $q$ appears in the final result above because the total range of the angular coordinate around the singular points on the universal cover is $\Delta \theta=2 \pi q$. We should also comment that implicitly we have used the fact that the integrand dies off sufficiently rapidly as $|\omega| \rightarrow \infty$ so that the potential boundary contribution coming from large $\omega$ actually vanishes.

Now according to the AdS/CFT dictionary, the above on-shell action can be interpreted as giving the leading saddle-point approximation of the partition function of the boundary theory evaluated on the universal cover of the orbifold, i.e., $Z_{q}=\exp \left(-I_{\text {tot }}\right)$. Now combining eqs. (3.2) and (3.3), we find

$$
S_{q}=\frac{1}{q-1}\left(q \log Z_{1}-\log Z_{q}\right)
$$

Since $Z_{1}$ is the partition function of the original (un-replicated) CFT in flat space, the corresponding holographic calculation would compute the on-shell gravity action (C.18) evaluated on empty $\mathrm{AdS}_{3}$ space in the usual Poincaré coordinates. However, using the prescription outlined here and in appendix $\mathbf{Q}$, this gravity action evaluates to zero and so $Z_{1}=1$. Therefore our leading semi-classical result for the Rényi entropy follows simply from eq. (3.21):

$$
S_{q}=\frac{1}{q-1} I_{\mathrm{tot}}=\frac{c}{6}\left(1+\frac{1}{q}\right) \ln \left(\frac{v_{2}-v_{1}}{\delta}\right)
$$

which again matches precisely with the expected result (1.5) for the Rényi entropy of a two-dimensional CFT. 


\subsection{UV regulator geometry}

In appendix [, we give a detailed discussion of the UV regulator surface that is implicit in the previous holographic calculations. Of course, one component of this surface is given as usual by the radial cut-off $\rho=\rho_{\min }=\delta^{2} / L^{2}$. However, there are two additional caps which keep the bulk integration away from the singularities in the boundary metric at $\omega=v_{2}$ and $\omega=v_{1}$. Hence we can think of the full regulator surface as being composed of these three components. This choice of an 'unusual' regulator surface is in fact the key to our gravitational calculation which gives a holographic translation of the determination of the Rényi entropy using twist operators discussed in section 3.1. That is, with $d=2$, the bulk geometry is simply empty $\mathrm{AdS}_{3}$ space. Hence the main effect of choosing coordinates with FG gauge (3.13) is to alter the UV cutoff surface near the $\mathrm{AdS}_{3}$ boundary relative to the 'constant radius' cut-off surface in Poincaré coordinates. Hence the interesting physical results in the previous section are coming from this 'unusual' regulator surface whose choice is motivated by the problem of calculating the Rényi entropy. In particular, this choice is made so that (to leading order in $\delta / L)$ the induced metric of the cut-off surface coincides with the boundary CFT metric in the conformal frame of interest.

This observation implies that we could have obtained the same result (3.23) in section 3.2 through the following route: We describe the $\mathrm{AdS}_{3}$ bulk with conventional Poincaré coordinates,

$$
d s^{2}=\frac{L^{2}}{\xi^{2}}\left(d \xi^{2}+d z d \bar{z}\right),
$$

but choose a nontrivial cut-off surface

$$
\xi=\frac{\delta}{L} e^{-\phi(z, \bar{z})}, \quad \text { where } \quad e^{\phi} \equiv q\left(v_{2}-v_{1}\right) \frac{|z|^{q-1}}{\left|z^{q}-1\right|^{2}} .
$$

Here we are following the analysis of [47] - see also appendix Q. With this choice, the induced metric on this cut-off surface has the same conformal factor appearing in eq. (3.5). Note that the factor $e^{-\phi}$ diverges as $z \rightarrow 0, \infty$. Hence we would introduce additional components to regulator surface which impose $|z|>\delta / L$ and $|z|<L / \delta$. These additional surfaces would correspond to the two caps which keep the bulk integration away from the twist operators in the boundary metric.

In fact, using the above approach, the on-shell gravity action evaluates to

$$
I_{\text {tot }}=-\frac{c}{48 \pi} \int d z d \bar{z}(\partial \phi \bar{\partial} \phi-4 \partial \bar{\partial} \phi)
$$

In the limit $\delta \rightarrow 0$, the above result is robust against changes in the choice of cut-off surface (3.25) which are sub-leading in $\delta / L$. This result is in keeping with the idea 
that a Weyl rescaling will shift the action of any $d=2$ CFT action by an expression proportional to the Liouville action, where the Liouville field takes the value of the Weyl factor [58. In the above expression, the first term is precisely the kinetic term of the Liouville field $\phi$. This result was also noted in [47], despite some small discrepancy in the choice of surface terms. At first sight, eq. (3.26) does not appear identical to the computation in FG coordinates, unless the second order derivatives of $\phi$ vanishes. However, as is noted in [43], the key non-vanishing contribution that is responsible for the final answer is in fact the kinetic term, whereas the rest drops out on careful regularization. For our purpose, $R_{(0)}=2 \partial \bar{\partial} \phi=0$ within the integration region, and indeed inserting the explicit form of $\phi$ into (3.26), we find agreement with (3.21), as is required by conformal symmetry.

In passing, let us comment on the black hole geometries appearing in section 2. The analog of the action calculation applied here would be to first evaluate the Euclidean gravitational action $I_{\mathrm{E}}$ for the topological black holes (2.1). Then interpreting this result in terms of the free energy of the dual thermal ensemble, i.e., $F(T)=T I_{\mathrm{E}}(T)$, the Rényi entropy would be calculated using eq. (1.9). Implicitly the regulator surface in this action calculation has distinct components. Of course, there would be the standard radial cut-off surface at $r=L^{2} / \delta$. However, as discussed after eq. (2.8), we must also impose an 'IR cut-off' in the radial integration across the hyperbolic geometry foliating the AdS geometry. That is, we will have a new cut-off surface near where these surfaces approach the asymptotic AdS boundary. As we will see from the discussion in the following section, these new surfaces are analogous to the caps above which restrict the bulk integration from approaching the position of the twist operators in the boundary metric.

\section{Twist operators and Thermal ensembles}

In the previous sections, we presented two holographic calculations of Rényi entropy, each of which was motivated by a distinct approach to calculating in the boundary CFT. We would now like to compare the latter two approaches and in fact, with closer examination, we find that they are closely related. To begin let us consider eq. (3.3) where one evaluates $\operatorname{tr}\left[\rho_{V}^{q}\right]=Z_{q} / Z_{1}^{q}$. Here $Z_{q}$ denotes the path integral of the $q$-fold replicated theory on the orbifold generated by the insertion of twist operators. The factor of $Z_{1}$ is simply the vacuum partition function of the original theory, which is used to normalize the result. Now we consider eq. (1.8) where one instead evaluates $\operatorname{tr}\left[\rho_{V}^{q}\right]=Z\left(T_{0} / q\right) / Z\left(T_{0}\right)^{q}$. Here the factor $Z\left(T_{0}\right)$ corresponds again to the vacuum

partition function, however, the conformal transformation introduced in [9] allows it to be interpreted as a thermal partition function. Hence it is natural to also associate 
the numerators in both of these expressions. That is, the thermal partition function $Z\left(T_{0} / q\right)$ should be equivalent to $Z_{q}$ in the previous calculation. Further just as the path integrals which define $Z_{1}$ and $Z\left(T_{0}\right)$ are related by a conformal transformation, it is natural to expect that the same should hold for $Z_{q}$ and $Z\left(T_{0} / q\right)$.

In fact, it is straightforward to establish these relations. Since the calculation with twist operators in section 3.1 explicitly referred to $d=2$, we start our discussion there. The thermal partition functions with be defined by a path integral over the CFT on a Euclidean background

$$
d s^{2}=d t_{\mathrm{E}}^{2}+d u^{2}
$$

where the Euclidean time coordinate is periodic with period $\Delta t_{\mathrm{E}}=q / T_{0}=2 \pi R q$ and $u \in\{-\infty, \infty\}$. Now for convenience, let us introduce the complex coordinate $\sigma=\left(u+i t_{\mathrm{E}}\right) / R$, which is then periodic along the imaginary axis with $\sigma=\sigma+2 \pi i q$. We can map the $\sigma$-cylinder to the flat $z$-plane with the conformal transformation: $z=\exp [\sigma / q]$. Note that we have included the factor of $1 / q$ in the exponent to ensure that this mapping is one-to-one between the $\sigma$-cylinder and the $z$-plane. However, let us now combine this transformation with eq. (3.4) which relates $z$ and $\omega$ in the twist operator calculation. The combined transformation becomes

$$
e^{\sigma}=\frac{\omega-v_{1}}{\omega-v_{2}}
$$

Naively, this mapping is independent of $q$, however, we must remember that there is an implicit $q$ dependence in the periodicity of $\sigma$. Therefore, this transformation (4.2) provides a conformal mapping from the $\left(u, t_{\mathrm{E}}\right)$-cylinder, which defines the thermal partition function at temperature $T=T_{0} / q$, to the $q$-fold universal cover of $\omega$-plane, which defines the partition function with twist operators at $\omega=v_{2}$ and $\omega=v_{1}$. Of course, with the choice $q=1$, we simply have a one-to-one mapping from $\left(u, t_{\mathrm{E}}\right)$-cylinder to the $\omega$-plane. Hence in general, we may equate $Z_{q}=Z\left(T_{0} / q\right)$ and $Z_{1}=Z\left(T_{0}\right)$ because the underlying CFT path integrals are simply related by conformal transformations.

Having made the desired identifications in $d=2$, let us now turn our attention to higher dimensions. As noted above, it seems straightforward to extend the replica trick to higher dimensional theories. However, this yields a rather formal definition of twist operators for higher dimensions and in practice beyond $d=2$, the construction (and properties) of these operators is not well understood - however, see [26, 40. In $d$ dimensions, a twist operator $\sigma_{q}$ would be some $(d-2)$-dimensional surface operator which again introduces a branch cut in the path integral over a $q$-fold replicated theory. Note that for $d>2$, we would not have distinct operators $\sigma_{ \pm q}$ to open and close the branch cuts. For example, in the problem of interest, the entangling surface is an $S^{d-2}$ 
and so there would be a single operator $\sigma_{q}$ which resides on this surface to open a branch cut over the ball on the interior.

Having introduced the notion of a twist operator for $d>2$, let us proceed with the consideration of $Z\left(T_{0} / q\right)$. In fact, the conformal mapping analogous to eq. (4.2) is discussed in 9]. However, the discussion is presented for Minkowski signature backgrounds and so we adapt their analysis to Euclidean signature. With $Z\left(T_{0} / q\right)$ in $d$ dimensions, we are considering a thermal ensemble at temperature $T=T_{0} / q$ on the background $R \times H^{d-1}$. The partition function can be evaluated by path integral on the Euclidean background $S^{1} \times H^{d-1}$

$$
d s^{2}=d \tau_{\mathrm{E}}^{2}+R^{2}\left(d u^{2}+\sinh ^{2} u d \Omega_{d-2}^{2}\right)
$$

where the Euclidean time coordinate has period $\Delta \tau_{\mathrm{E}}=q / T_{0}=2 \pi R q$. In the following, it will be convenient to introduce complex coordinates:

$$
\sigma=u+i \tau_{\mathrm{E}} / R \quad \text { and } \quad \omega=r+i t_{\mathrm{E}} .
$$

The latter will be used to describe a conformally mapped geometry below. Note that both $u$ and $r$ are radial coordinates, we must have $\operatorname{Re}(\sigma)=u>0$ and $\operatorname{Re}(\omega)=r>0$. Now, with the first of these new coordinates, the above metric (4.3) can be written

$$
d s^{2}=R^{2}\left(d \sigma d \bar{\sigma}+\sinh ^{2}\left(\frac{\sigma+\bar{\sigma}}{2}\right) d \Omega_{d-2}^{2}\right) .
$$

Now we make the coordinate transformation

$$
e^{-\sigma}=\frac{R-\omega}{R+\omega}
$$

Since we are considering $d \geq 3$ there is no guarantee that this holomorphic change of coordinates will result in a conformal transformation. However, one can readily verify the above metric (4.5) becomes

$$
\begin{aligned}
d s^{2} & =\Omega^{2}\left[d \omega d \bar{\omega}+\left(\frac{\omega+\bar{\omega}}{2}\right)^{2} d \Omega_{d-2}^{2}\right] \\
& =\Omega^{2}\left[d t_{\mathrm{E}}^{2}+d r^{2}+r^{2} d \Omega_{d-2}^{2}\right]
\end{aligned}
$$

where

$$
\Omega=\frac{2 R^{2}}{\left|R^{2}-\omega^{2}\right|} .
$$

Hence, after eliminating the conformal factor $\Omega^{2}$ in the second line of eq. (4.7), we recognize that the final line element is simply the metric on $d$-dimensional flat space 
$R^{d}$. However, we must again pay special attention to the identification of the $\sigma$. In particular, since $\Delta \tau_{\mathrm{E}}=q / T_{0}=2 \pi R q$, we must identify $\sigma=\sigma+2 \pi i q$. Therefore the transformation (4.6) actually maps the original background $S^{1} \times H^{d-1}$ to a $q$-fold cover of $R^{d}$ with an orbifold singularity on precisely the $(d-2)$-dimensional sphere given by $r=R$. Hence evaluating the path integral on the new geometry would yield precisely the partition function $Z_{q}$ of a $q$-fold replicated theory with an $S^{d-2}$ twist operator inserted at $r=R$. Hence as long as we are considering a conformal field theory, we may equate $Z_{q}=Z\left(T_{0} / q\right)$ because the path integrals are simply related by a conformal transformation. Of course, with the choice $q=1$, we again have a simple one-to-one mapping from $S^{1} \times H^{d-1}$ to $R^{d}$.

Turning now to our holographic calculations, we evaluate the partition functions above in the semi-classical approximation by evaluating the on-shell gravitational action for a particular saddle-point. For both $Z_{1}$ and $Z\left(T_{0}\right)$, the corresponding saddle-point is simply the AdS vacuum evaluated with a conventional UV regulator. The latter description applies equally for $d \geq 3$ or for $d=2$. In the case of $d=2$, the saddlepoint corresponding to $Z_{q}$ and $Z\left(T_{0} / q\right)$ is again $\mathrm{AdS}_{3}$ but an unconventional choice of regulator surface is chosen, as discussed in section 3.3. Now in higher dimensions (i.e., with $d \geq 3$ ), the saddle-point dual to $Z\left(T_{0} / q\right)$ is a topological black hole (2.1) with an appropriately chosen horizon temperature. This highlights a basic difference for higher dimensions. In $d=2$ any boundary metric is (locally) conformally flat and so the corresponding bulk is always just $\mathrm{AdS}_{3}$. In higher dimensions, we can rarely transform to a flat metric and so generically our bulk will not just be the $\mathrm{AdS}_{d+1}$ vacuum. Rather in higher dimensions, we have to find new smooth solutions of the gravitational equations

with appropriate boundary conditions. In particular, to evaluate $Z_{q}$, we would want a new bulk solution where the conical singularity introduced by the twist operator arises only at the boundary, i.e., it would only appear in the induced metric on UV regulator surface, as discussed in section 3.3. Now in principle, the black hole geometries provide precisely the desired bulk space and one is only required to make a coordinate transformation in the bulk which would implement the conformal transformation (4.6) on the boundary. Unfortunately, this still seems to be quite a challenging exercise. However, it does appear to be less formidable than constructing the bulk geometry without the insight of equating $Z_{q}=Z\left(T_{0} / q\right)$.

\subsection{Twist operators and Black holes}

In this section, we wish to apply the insights above to our holographic models in section 2. In particular, we will use our holographic calculations to evaluate the scaling dimension of the twist operators for the boundary theory in general dimensions. 
As a warm-up exercise, we will apply holography to reproduce the expression (3.11) for the total scaling dimension of twist operators in a two-dimensional CFT. In the case of $d=2$, eqs. (2.1) and (2.13) describe a black hole solution for three-dimensional Einstein gravity

$$
d s^{2}=-\frac{r^{2}-r_{\mathrm{H}}^{2}}{R^{2}} d t^{2}+\frac{L^{2} d r^{2}}{r^{2}-r_{\mathrm{H}}^{2}}+\frac{r^{2}}{R^{2}} d \hat{u}^{2},
$$

where we have modified slightly the normalization of the spatial coordinates. With $d=2$, rather than a hyperbolic geometry, the spatial CFT geometry is simply a line and so we have chosen a normalization such that the boundary metric (2.2) becomes simply $d s_{\infty}^{2}=-d t^{2}+d \hat{u}^{2}$. Note that with $d=2$, the formula for the temperature (2.17) simplifies to $T=T_{0} x$ where $T_{0}=1 /(2 \pi R)$ and $x=r_{\mathrm{H}} / L$ as was used throughout section 2. As is already evident from these expressions, the solution of eq. (2.19) is simply $x_{q}=1 / q$. Now we should note that this solution (4.9) is nothing other than the spinless BTZ black hole [57] but without any orbifolding along the spatial direction to preserve the noncompact boundary. Without the latter orbifolding, it is also true that this solution is simply $\mathrm{AdS}_{3}$ space written in unconventional coordinates. Before proceeding further, it is convenient to go to Euclidean signature with which the threedimensional black hole metric becomes

$$
d s^{2}=L^{2} \frac{d r^{2}}{r^{2}-r_{\mathrm{H}}^{2}}+\frac{r^{2}-r_{\mathrm{H}}^{2}}{R^{2}} d t_{\mathrm{E}}^{2}+\frac{r^{2}}{R^{2}} d \hat{u}^{2} .
$$

Now adapting well-known results for the BTZ black hole 48], we use the following bulk coordinate transformation to take this Euclidean black hole metric back into the Poincaré metric (3.24):

$$
z=\frac{\sqrt{r^{2}-r_{\mathrm{H}}^{2}}}{r} \exp \left[x\left(\frac{\hat{u}+i t_{\mathrm{E}}}{R}\right)\right], \quad \xi=\frac{r_{\mathrm{H}}}{r} \exp \left[x \frac{\hat{u}}{R}\right],
$$

where as before $x=r_{\mathrm{H}} / L$. The following is simplified by using a the complex coordinate $\sigma=\left(u+i t_{\mathrm{E}}\right) / R$, which was introduced in the previous section. Note that if we substitute $x=1 / q$ in eq. (4.11), then in the boundary limit, i.e., with $r \rightarrow \infty, z \simeq \exp [\sigma / q]$ and so this bulk coordinate transformation (4.11) implements the first conformal mapping considered in the previous section.

For our present purposes, the essential information provided by the black hole solution is the thermal energy density, which can be read off with the conventional holographic map

$$
T_{\sigma \sigma}(T)=\frac{\pi}{12} c T^{2}
$$


Now the conformal map between the $\sigma$ and $\omega$ coordinates is given above by eq. (4.2). Hence the stress tensor is mapped between these two conformal frames as

$$
\begin{aligned}
T_{\omega \omega} & =\sigma^{\prime}(\omega)^{2} T_{\sigma \sigma}\left(T_{0} / q\right)-\frac{c}{24 \pi}\{\sigma, \omega\} \\
& =-\frac{c}{48 \pi}\left(1-\frac{1}{q^{2}}\right) \frac{\left(v_{2}-v_{1}\right)^{2}}{\left(\omega-v_{2}\right)^{2}\left(\omega-v_{1}\right)^{2}} .
\end{aligned}
$$

Hence we have reproduced the expected result for the correlator of the stress tensor with a pair of twist operators, as given in eq. (3.7). Hence following the same logic presented in section 3.1, we can infer that the scaling dimension of the twist operators is given by eq. (3.11).

Before concluding, let us comment on the anomalous contribution given the Schwarzian in eq. (4.13). Recall that in general the Schwarzian is given by eq. (3.8) and so depends only on the form of the coordinate transformation. If we evaluate this expression for the present transformation (4.2), we note that it can be expressed as

$$
\{\sigma, \omega\}=24 \pi c \sigma^{\prime}(\omega)^{2} T_{\sigma \sigma}\left(T_{0}\right) .
$$

One can also infer this relation because if we insert $q=1$ into eq. (4.13), the result vanishes.

Now we would now like extend the previous discussion, which focussed on $d=2$ and used the three-dimensional bulk black hole (4.9), to higher dimensions and determine $h_{q}$ for the general holographic models of section 2. As background for this analysis in higher dimensions, we first consider the following result which would apply for any CFT: Imagine making an insertion of the stress tensor in the presence of a planar twist operator $\sigma_{q}$. Let us describe the $R^{d}$ background with Cartesian coordinates $x^{\mu}=\left\{x^{i}, i=0,1 ; x^{a}, a=2, \ldots, d-1\right\}$. The $(d-2)$-dimensional twist operator will be positioned at $x^{0}=0=x^{1}$ while it extends throughout all of the $x^{a}$ directions. We insert the stress tensor at $x^{\mu}=\left\{\alpha^{i}, \beta^{a}\right\}$ and define $\alpha=\sqrt{\left(\alpha^{1}\right)^{2}+\left(\alpha^{2}\right)^{2}}$. Then symmetry ${ }^{12}$ dictates that the singularity in the corresponding correlator takes the following form

$$
\begin{aligned}
\left\langle T_{a b} \sigma_{q}\right\rangle & =-\frac{h_{q}}{2 \pi} \frac{\delta_{a b}}{\alpha^{d}}, \quad\left\langle T_{a i} \sigma_{q}\right\rangle=0, \\
\left\langle T_{i j} \sigma_{q}\right\rangle & =\frac{h_{q}}{2 \pi} \frac{(d-1) \delta_{i j}-d n_{i} n_{j}}{\alpha^{d}} .
\end{aligned}
$$

\footnotetext{
${ }^{12}$ The translation and rotation symmetries dictate the basic geometric structure in the following and the relative normalization of various contributions is fixed by imposing $\left\langle T_{\mu}^{\mu} \sigma_{q}\right\rangle=0=\nabla^{\mu}\left\langle T_{\mu \nu} \sigma_{q}\right\rangle$. Let us also note here that the correlators in eq. (4.15) are implicitly normalized by dividing out by $\left\langle\sigma_{q}\right\rangle$ but we left this implicit to avoid the clutter.
} 
where $n^{i}=\alpha^{i} / \alpha$ is the unit vector point directed orthogonally from the twist operator to the $T_{\mu \nu}$ insertion. These correlators (4.15) define the coefficient $h_{q}$ which is commonly called the scaling dimension of $\sigma_{q}$, since its appearance here is analogous to that of the scaling dimension of a local primary operator. We are assuming that $T_{\mu \nu}$ corresponds to the total stress tensor for the entire $q$-fold replicated CFT, i.e., $T_{\mu \nu}$ is inserted on all $q$ sheets of the universal cover. Then if one reduces these expressions to $d=2$, one finds that the present definition for $h_{q}$ matches precisely with those given in section 3.1. Hence for $d=2, h_{q}$ is the total scaling dimension given in eq. (3.11).

As noted above, the essential information that we must extract from the topological black holes is the stress-energy of the thermal ensemble on $R \times H^{d-1}$ or rather the Euclidean CFT lives on the background $S^{1} \times H^{d-1}$. However, on general grounds, we know the expectation value of the stress tensor is restricted to take the form

$$
T^{\mu}{ }_{\nu}=\operatorname{diag}(-\mathcal{E}, p, \cdots, p),
$$

with $\mathcal{E}$ and $p$ constants - this form is independent of the choice of either Minkowski or Euclidean signature. Further for a conformal field theory the trace of this expression must vanish ${ }^{13}$ and so in eq. (4.16), we will find

$$
p=\mathcal{E} /(d-1) .
$$

In particular then, we will use the black hole solution in higher dimensions to determine the energy density $\mathcal{E}(T)$.

To connect this thermal energy density to the correlator with the twist operator, we must conformally map the $S^{1} \times H^{d-1}$ background to flat metric on $R^{d}$ using the transformation in eq. (4.6). As discussed above, when the temperature is tuned to $T=T_{0} / q$, this mapping actually takes the original background to a $q$-fold cover of $R^{d}$ with an orbifold singularity on the sphere given by $r=R$. Now in higher dimensions, a conformal mapping transforms the stress tensor as

$$
T_{\alpha \beta}=\Omega^{d-2} \frac{\partial X^{\mu}}{\partial x^{\alpha}} \frac{\partial X^{\nu}}{\partial x^{\beta}}\left(T_{\mu \nu}\left(T_{0} / q\right)-\mathcal{A}_{\mu \nu}\right),
$$

where $\mu, \nu$ and $\alpha, \beta$ denote indices on the flat geometry and $S^{1} \times H^{d-1}$, respectively. Since the stress tensor is not a primary operator, in general, the transformation (4.18)

\footnotetext{
${ }^{13}$ One may worry that for even $d$ that the trace anomaly will lead to a nonvanishing trace. However, one can readily verify that in fact the trace anomaly vanishes for the background geometry $R \times H^{d-1}$. In particular, note that the Euler density vanishes because the background is the direct product of two lower dimensional geometries. Further this background is conformally flat and so any conformal invariants also vanish.
} 
includes an anomalous contribution, which we denoted $\mathcal{A}_{\mu \nu}$. This contribution is the higher dimensional analog of the Schwarzian appearing in eq. (4.13). Again we note that this anomalous term depends entirely on the form of the transformation. Hence it can be fixed by observing that with $q=1$, eq. (4.6) actually yields a one-to-one mapping from $S^{1} \times H^{d-1}$ to $R^{d}$. In the absence of any singularities, i.e., without the insertion of a twist operator, $T_{\alpha \beta}$ is simply the vacuum expectation value of the stress energy in $R^{d}$ and hence it must vanish. Since the left-hand side of eq. (4.18) vanishes with $q=1$, we must have $\mathcal{A}_{\mu \nu}=T_{\mu \nu}\left(T_{0}\right)$. Hence the transformation (4.18) becomes

$$
T_{\alpha \beta}=\Omega^{d-2} \frac{\partial X^{\mu}}{\partial x^{\alpha}} \frac{\partial X^{\nu}}{\partial x^{\beta}}\left(T_{\mu \nu}\left(T_{0} / q\right)-T_{\mu \nu}\left(T_{0}\right)\right),
$$

Recall that the required conformal factor $\Omega$ is given in eq. (4.8).

Now the conformal mapping (4.6) generates a spherical twist operator while scaling dimension $h_{q}$ was defined by the correlator with a planar twist operator. However, if the insertion of the stress tensor approaches very close to the spherical twist operator, the leading singularity will take the form given in eq. (4.15). Hence we can still easily identify $h_{q}$ in this limit. To simplify the analysis, we will consider evaluating $T_{\alpha \beta}$ at $t_{\mathrm{E}}=0$ and $r=R-\alpha$ with $\alpha \ll R$ (as well as some fixed angles). With this choice, one finds that various relevant expressions simplify. For example, from eq. (4.8), we find $\Omega \simeq R / \alpha$ and using eq. (4.6), we can show that $\frac{\partial u}{\partial t_{\mathrm{E}}} \simeq 0$ and $\frac{\partial \tau_{\mathrm{E}}}{\partial t_{\mathrm{E}}} \simeq R / \alpha$. Given the last two expressions, one finds that evaluating eq. (4.19) for $\alpha=t_{\mathrm{E}}=\beta$ yields

$$
\begin{aligned}
T_{t_{\mathrm{E}} t_{\mathrm{E}}} & =\Omega^{d-2}\left(\frac{\partial \tau_{\mathrm{E}}}{\partial t_{\mathrm{E}}}\right)^{2}\left(T_{\tau_{\mathrm{E}} \tau_{\mathrm{E}}}\left(T_{0} / q\right)-T_{\tau_{\mathrm{E}} \tau_{\mathrm{E}}}\left(T_{0}\right)\right)+\cdots \\
& =\left(\frac{R}{\alpha}\right)^{d}\left(\mathcal{E}\left(T_{0}\right)-\mathcal{E}\left(T_{0} / q\right)\right)+\cdots
\end{aligned}
$$

Now this result should be compared to the $i=t_{\mathrm{E}}=j$ component in eq. (4.15), i.e., $\left\langle T_{t_{\mathrm{E}} t_{\mathrm{E}}} \sigma_{q}\right\rangle=-h_{q}(d-1) /\left(2 \pi \alpha^{d}\right)$. However, we note that the latter expectation value involves the total stress tensor for the entire $q$-fold replicated CFT. However, it is clear that eq. (4.20) corresponds to an insertion of $T_{\mu \nu}$ in a single sheet of the universal cover and hence we must multiply by an extra factor of $q$ to compare the two expressions. The final result is

$$
h_{q}=2 \pi \frac{q R^{d}}{d-1}\left(\mathcal{E}\left(T_{0}\right)-\mathcal{E}\left(T_{0} / q\right)\right)
$$

We are now almost ready to evaluate these weights for the holographic models considered in section 2. The simplest way to connect the above expression to the results in this section is to use the first law of thermodynamics, i.e., $d E=T d S$. Then 
one may write

$$
\begin{aligned}
\mathcal{E}\left(T_{0}\right)-\mathcal{E}\left(T_{0} / q\right) & =\frac{1}{R^{d-1} V_{\Sigma}} \int_{x_{q}}^{1} T(x) \frac{d S}{d x} d x \\
& =\frac{1}{R^{d-1} V_{\Sigma}}\left(T_{0} S\left(T_{0}\right)-\frac{T_{0}}{q} S\left(T_{0} / q\right)-\frac{q-1}{q} T_{0} S_{q}\right) .
\end{aligned}
$$

Here we have used $\mathcal{E}=E /\left(R^{d-1} V_{\Sigma}\right)$ to define the energy density on the hyperbolic plane $H^{d-1}$. The second expression is derived by integrating by parts in the first line and substituting eq. (2.6) for the remaining integral. Combining eqs. (4.21) and (4.21) then yields

$$
h_{q}=\frac{1}{(d-1) V_{\Sigma}}\left(q S\left(T_{0}\right)-S\left(T_{0} / q\right)-(q-1) S_{q}\right)
$$

where we have simplified the pre-factor above using $T_{0}=1 /(2 \pi R)$.

Hence we may now apply our results in section 2 to calculate the scaling dimension $h_{q}$. First using the expressions of Einstein gravity in section 2.1, we find

$$
h_{q}=\pi\left(\frac{L}{\ell_{\mathrm{P}}}\right)^{d-1} q x_{q}^{d-2}\left(1-x_{q}^{2}\right),
$$

where $x_{q}$ is related to $q$ through eq. (2.19). Next using the results of section 2.2, we have for the boundary CFT's dual to GB gravity in arbitrary dimensions

$$
h_{q}=\frac{1}{4} \Gamma\left(\frac{d}{2}\right) \pi^{1-\frac{d}{2}} q x_{q}^{d-4}\left(x_{q}^{2}-1\right)\left((d-3)\left(x_{q}^{2}-1\right) a_{d}^{*}+\left(d-3-(d+1) x_{q}^{2}\right) \widetilde{C}_{T}\right)
$$

where $x_{q}$ is now given by eq. (2.39). Interestingly, we find tremendous simplicity in the $q \rightarrow 1$ limit:

$$
\left.\partial_{q} h_{q}\right|_{q=1}=\frac{2}{d-1} \pi^{1-\frac{d}{2}} \Gamma(d / 2) \widetilde{C}_{T}
$$

Repeating the calculation once more for quasi-topological gravity with $d=4$, using the formulae from section 2.3, we find that $h_{q}$ has a complicated dependence both central charges, $a$ and $c$, as well as $t_{4}$ :

$$
h_{q}=\frac{q a}{4 \pi x_{q}^{2}}\left(x_{q}^{2}-1\right)\left(x_{q}^{4}\left(1-5 \frac{c}{a}-10 \frac{c}{a} t_{4}\right)-x_{q}^{2}\left(1-\frac{c}{a}-8 \frac{c}{a} t_{4}\right)+2 \frac{c}{a} t_{4}\right)
$$

where eq. (2.57) determines the value of $x_{q}$. Nevertheless, we once again notice that the derivative $\left.\partial_{q} h_{q}\right|_{q=1}$ is simply proportional to the central charge $c$ and independent of $a$ and $t_{4}$ :

$$
\left.\partial_{q} h_{q}\right|_{q=1}=\frac{2 c}{3 \pi}
$$


which matche precisely with eq. (4.26) for GB gravity with $d=4$.

Hence in general, these scaling weights have a complicated nonlinear dependence on the various parameters characterizing the boundary theory, including $t_{4}$ in the last example. Note that although the result in, e.g., eq. (4.25) appears linear in both $\widetilde{C}_{T}$ and $a_{d}^{*}$, since $x_{q}$ is determined as the root of eq. (2.39), it introduces a complicated nonlinear dependence on these parameters. However, we find a remarkable simplification in evaluating $\partial_{q} h_{q}$ in the limit $q \rightarrow 1$ with the result being proportional to the central charge $\widetilde{C}_{T}{ }^{14}$ This is somewhat analogous to the simplification found for the Rényi entropy in this limit, i.e., the entanglement entropy $S_{1}$ has a simple form being proportional to the central charge $a$ and independent of any other CFT parameters.

\section{Eigenvalue Spectrum}

In this section, we begin to explore what information can be inferred about the eigenvalue spectrum of the reduced density matrix using our holographic results for the Rényi entropy. Of course, having access to all of the Rényi entropies $S_{q}$ for arbitrary $q$ gives us far more information about this spectrum than is contained in the entanglement entropy $S_{1}$ alone. In fact, it was shown in [4] that the full spectrum of eigenvalues can be calculated by knowing $\operatorname{tr}\left[\rho_{V}^{q}\right]$ for only integer $q$.

First let us recall that the limit of infinite $q$ yields

$$
S_{\infty}=\lim _{q \rightarrow \infty} S_{q}=-\log \lambda_{1},
$$

where $\lambda_{1}$ is the largest eigenvalue of $\rho_{V}$. This result is usually discussed in the context of a quantum mechanical system with a discrete spectrum. In the present context, we are studying holographic CFT's and so can expect there may be various complications. First of all, we are evaluating the entire expression $S_{q}$ above, i.e., this expression does not apply for the universal contribution to $S_{q}$. Hence we must think of $S_{q}$ for the boundary CFT in the explicit presence of the short distance cut-off $\delta$, Hence $S_{q}$ is dominated by the leading contribution in $V_{\Sigma}$, which for $d>2$ is proportional to $\mathcal{A} / \delta^{d-2}$ where $\mathcal{A}$ is the area of the entangling surface. Therefore eq. (5.1) indicates that the largest eigenvalue (and hence all the eigenvalues) are approaching zero with

$$
\lambda_{1} \propto \exp \left[- \text { const. } \times a_{d}^{*} \times \mathcal{A} / \delta^{d-2}\right]
$$

as $\delta \rightarrow 0$. Of course, our results indicate that the (positive) constant prefactor in the exponent above will in general have a complicated dependence on various CFT

\footnotetext{
${ }^{14}$ Of course, by itself, the scaling dimension vanishes in the limit $q \rightarrow 1$, i.e., $h_{1}=0$, since there is no twist operator at $q=1$.
} 
parameters. However, we have explicitly included the factor $a_{d}^{*}$ to remind ourselves that even with a fixed cut-off $\delta$, the exponent is large in the classical gravity limit since it grows with the central charge $a_{d}^{*}$. For example, in the well-known case where the boundary CFT is $d=4$ super-Yang-Mills, this factor grows like $N_{c}^{2}$. While this holographic result may seem rather counter-intuitive, we find an analogous result in computing $S_{q}$ for free fields living on a hyperbolic waveguide geometry [50].

To proceed further, we will consider expanding the Rényi entropy for large $q$. As a warm-up exercise, let us assume that the eigenvalue spectrum is discrete, in which case we have

$$
\begin{aligned}
S_{q} & =\frac{1}{1-q} \log \operatorname{tr}\left[\rho_{V}^{q}\right] \\
& =\frac{1}{1-q} \log \left[d_{1} \lambda_{1}^{q}+d_{2} \lambda_{2}^{q}+\cdots\right] .
\end{aligned}
$$

In the second line, we have an explicit sum over the eigenvalues $\lambda_{i}$ of the density matrix but we also allow the various eigenvalues to be degenerate with multiplicity $d_{i}$. Now with large but finite $q$, we can expand the last expression as

$$
\begin{aligned}
S_{q} & \simeq-\frac{1}{q}\left(1+\frac{1}{q}\right)\left[q \log \lambda_{1}+\log d_{1}+\log \left(1+\frac{d_{2}}{d_{1}}\left(\frac{\lambda_{2}}{\lambda_{1}}\right)^{q}\right)\right] \\
& \simeq-\log \lambda_{1}-\frac{1}{q} \log \left(d_{1} \lambda_{1}\right)+\mathcal{O}\left[\frac{1}{q^{2}}, \frac{1}{q}\left(\frac{\lambda_{2}}{\lambda_{1}}\right)^{q}\right]
\end{aligned}
$$

In this case, the next-to-leading term at order $1 / q$ tells us about the degeneracy $d_{1}$ of the largest eigenvalue. Note also, that this large- $q$ expansion contains contributions of the form $\frac{1}{q}\left(\frac{\lambda_{2}}{\lambda_{1}}\right)^{q}$, which are nonanalytic in $1 / q$.

Let us now evaluate $\lambda_{1}$ and $d_{1}$ from large- $q$ expansion of the holographic Rényi entropy in the simplest case of Einstein gravity discussed in section 2.1. From eqs. (2.19) and (2.20), we have

$$
\begin{aligned}
& x_{q}=x_{\infty}+\frac{1}{d q}+\mathcal{O}\left(\frac{1}{q^{2}}\right) \\
& S_{q}=2 \pi V_{\Sigma}\left(\frac{L}{\ell_{\mathrm{P}}}\right)^{d-1}\left[\left(1-\frac{d-1}{d-2} x_{\infty}^{d}\right)+\frac{1}{q}\left(1-\frac{d-1}{d-2} x_{\infty}^{d}-x_{\infty}^{d-1}\right)+\mathcal{O}\left(\frac{1}{q^{2}}\right)\right]
\end{aligned}
$$

where $x_{\infty}=\sqrt{d(d-2)} / d$. In particular, this means that

$$
\lambda_{1}=\exp \left[-2 \pi V_{\Sigma}\left(\frac{L}{\ell_{\mathrm{P}}}\right)^{d-1}\left(1-\frac{d-1}{d-2} x_{\infty}^{d}\right)\right], \quad d_{1}=\exp \left[2 \pi V_{\Sigma}\left(\frac{L}{\ell_{\mathrm{P}}}\right)^{d-1} x_{\infty}^{d-1}\right]
$$


Note that the factor of $\left(L / \ell_{\mathrm{P}}\right)^{d-1}$ in the second exponent indicates the degeneracy $d_{1}$ is also growing as an exponential of the central charge of the boundary CFT. In the following, we show that the value of $d_{1}$ is related to the degeneracy of the ground state of CFT on $R \times H^{d-1}$. It is interesting to note that for $d=2$, the above holographic result states that $d_{1}=1$. The same result follows from the general form (1.5) of the Rényi entropy for $d=2$ CFT's since the leading coefficient precisely matches that for the $1 / q$ term. We also note that the latter result implies that the expansion of the Rényi entropy (5.4) for $d=2$ terminates at order $1 / q$. This will imply certain constraints on the structure of the spectral function, which in turn will allow us to evaluate it.

A further observation is that our holographic result yields an expansion (5.5) which appears to be completely analytic in the limit of large $q{ }^{15}$ In contrast, the general expansion in eq. (5.4) contains nonanalytic terms. However, it seems that the latter are a consequence of our simplifying assumption that the spectrum is discrete. Hence, instead one can consider the density matrix to have a smooth distribution $d(\lambda)$ of eigenvalues and then the Rényi entropy would become

$$
S_{q}=\frac{1}{1-q} \log \left[\int_{0}^{\lambda_{1}} d \lambda d(\lambda) \lambda^{q}\right] .
$$

While we leave the details as an exercise to the reader, one can easily show that this ansatz also leads to nonanalytic contributions in the large- $q$ expansion. Hence we are led to conclude that the eigenvalue spectrum must have both smooth and discrete components, i.e., $d(\lambda)$ must contain delta-functions supported on a discrete set of eigenvalues.

To provide evidence for the above structure and to facilitate further analysis, we continue by examining the spectral structure of the density matrix $\rho_{V}$ from the thermodynamic perspective, which is succinctly encoded in eq. (1.9). We will assume that there are no phase transitions in the system under consideration and so the free energy and its derivatives are continuous functions of the temperature T. Expanding eq. (1.9) near $q=\infty$ and using the thermodynamic identity $F^{\prime}(T)=-S(T)$ then yields

$$
\begin{aligned}
S_{q}=-\frac{F\left(T_{0}\right)-F(0)}{T_{0}}- & \frac{1}{q}\left(\frac{F\left(T_{0}\right)-F(0)}{T_{0}}+S(0)\right) \\
& -\frac{1}{q^{2}}\left(\frac{F\left(T_{0}\right)-F(0)}{T_{0}}+S(0)-\frac{1}{2} T_{0} F^{\prime \prime}(0)\right)+\mathcal{O}\left(1 / q^{3}\right)
\end{aligned}
$$

Again the leading term yields $-\log \lambda_{1}$ and so we have

$$
\lambda_{1}=\exp \left[\frac{F\left(T_{0}\right)-F(0)}{T_{0}}\right]=\frac{e^{-E_{1} / T_{0}}}{Z\left(T_{0}\right)},
$$

\footnotetext{
${ }^{15}$ Note that this may be an artifact of the limit of large central charge, i.e., the large $N_{c}$ limit, in the boundary CFT.
} 
where $E_{1}$ is the energy of the ground state. That is, from the thermal perspective, we see that $\lambda_{1}$ is indeed the largest eigenvalue of the thermal density matrix of the CFT at temperature $T_{0}$ on $R \times H^{d-1}$. Of course, this result is in complete agreement with the statement that there is a one-to-one correspondence between the eigenvalues and their degeneracies of the reduced density matrix $\rho_{V}$ and the thermal density matrix. Indeed, comparing to eq. (5.4), we next extract

$$
\log d_{1}=S(0)
$$

from the term of order $1 / q$ in eq. (5.8). That is, the degeneracy $d_{1}$ of the largest eigenvalue $\lambda_{1}$ equals to the degeneracy of the ground state of the thermal CFT. Substituting these identifications in eq. (5.8), we find entropy. Hence,

$$
S_{q}=-\log \lambda_{1}-\frac{1}{q} \log \left(d_{1} \lambda_{1}\right)-\frac{1}{q^{2}}\left(\log \left(d_{1} \lambda_{1}\right)-\frac{1}{2} T_{0} F^{\prime \prime}(0)\right)+\mathcal{O}\left(1 / q^{3}\right) .
$$

Again we observe that the expansions in eqs. (5.8) and (5.11) will be analytic in $1 / \beta=T_{0} / q$ as long as the free energy is analytic in the vicinity of vanishing temperature. Given our previous analysis, this again points to a distribution of eigenvalues that has both smooth and discrete components. Note that from the thermal perspective, it is natural to think in terms of energy eigenvalues and the thermodynamic spectral function $\rho(E)$. Hence let us consider an ansatz where $\rho(E)$ contains delta functions supported on some discrete set of energies $\left\{E_{i}, i=1, \ldots, i_{\max }\right\}$ as well as a smooth continuous distribution, i.e.,

$$
Z(\beta)=\sum_{i=1}^{i_{\max }} d_{i} e^{-\beta E_{i}}+\int_{E_{0}}^{\infty} \bar{\rho}(E) e^{-\beta E} d E,
$$

where $\bar{\rho}(E)$ is the smooth component of the spectral function and $\left\{d_{i}, i=1, \ldots, i_{\max }\right\}$ are the degeneracies of the discrete eigenvalues $E_{i}$. Without loss of generality, we are assuming the smooth component has support down to some $E_{0}$ with $E_{0} \geq E_{1}$. Note also that $\bar{\rho}\left(E_{0}\right) \neq 0$, since otherwise the free energy will be nonanalytic in the neighbourhood of $T=0$ and thus contradicts our holographic result.

Expanding in $1 / \beta$, yields to leading order

$$
F(\beta)=-\frac{1}{\beta} \log Z(\beta)=E_{1}-\frac{1}{\beta} \log d_{1}+\ldots
$$

This expansion agrees with (5.8) and in particular, as claimed before $S(T=0)=\log d_{1}$. Furthermore, if we expand to higher orders in the vicinity of $T=0$, we will encounter corrections proportional to $e^{-\beta\left(E_{i}-E_{0}\right)} / \beta$ with $i>1$, which are again nonanalytic in 
$1 / \beta$. While this sort of correction does not contradict any thermodynamic reasoning and it would seem to indicate that the free energy is nonanalytic in the neighbourhood of $T=0$ (or equivalently $q=\infty$ ). However, this would conflict with our holographic result, where only inverse powers of $q$, or alternatively $\beta$, are present in the expansion. Hence we proceed by investigating the consequences of demanding that these corrections are absent in keeping with holography. In particular, one immediate consequence is that $i_{\text {max }}=1$, namely there is only one delta function supported on the ground state of the CFT. Similarly, one can show that analyticity of $S_{q}$ near $q=\infty$ (i.e., our holographic restriction) requires $E_{0}=E_{1}$ as well as analyticity of $\bar{\rho}(E)$ in the vicinity of the ground state $E=E_{1}$.

Combining altogether, we have for the density of states

$$
\rho(E)=d_{1} \delta\left(E-E_{1}\right)+\bar{\rho}(E)
$$

with $\bar{\rho}(E)$ being analytic at $E_{1}$. Then expanding the resulting free energy for large $\beta$ then yields

$$
F(\beta)=-\frac{1}{\beta} \log Z(\beta)=E_{1}-\frac{1}{\beta} \log d_{1}-\frac{1}{\beta} \log \left(1+\frac{1}{d_{1} \beta} \sum_{n=0}^{\infty} \frac{\bar{\rho}^{(n)}\left(E_{1}\right)}{\beta^{n}}\right) .
$$

Comparing this result to eq. (5.11), we find by comparing the terms of order $1 / q^{2}$ :

$$
F^{\prime \prime}(0)=-2 \frac{\bar{\rho}\left(E_{1}\right)}{d_{1}} .
$$

Recall the thermodynamic identity $F^{\prime \prime}(T)=-C_{v} / T$ where $C_{v}$ is the heat capacity.

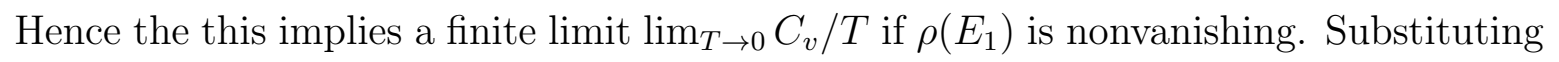
this result for the free energy (5.15) into eq. (1.9), we would find the large- $\beta$ expansion of the Rényi entropy. Then we could expand our holographic results for $S_{q}$ in e.g., eq. (2.38) around $q=\infty$ and by comparing with the two expansions, we can reconstruct the spectral function $\rho(E)$ in the neighbourhood of the ground state $E=E_{1}$.

Let us consider the case of $d=2$ (holographic) CFT's. In this case, the hyperbolic plane reduces to an infinite line and the free energy of the field theory is given by

$$
F(T)=-\frac{c}{6} \frac{T^{2}}{T_{0}} \log (2 R / \delta) .
$$

To get this result, one merely notes that for $d=2$, the Euclidean background $S^{1} \times R$ can be conformally mapped to the infinite flat plane, as discussed in section 4 . Therefore bearing in mind that the field theory is conformal, we deduce $F \sim T^{2} V_{\Sigma}(d=2) \sim$ $T^{2} \log (2 R / \delta)$ and the proportionality constant is fixed by requiring the thermal entropy 
derived from this expression to be equal the entanglement entropy, $S_{1}$. Comparing this expression with (5.15), yields

$$
d_{1}=1, \quad \bar{\rho}^{(n)}\left(E_{1}\right)=\frac{\bar{\rho}_{0}^{n+1}}{(n+1) !} \text { for } n \geq 0,
$$

where

$$
\bar{\rho}_{0}=\frac{c}{6 T_{0}} \log (2 R / \delta) .
$$

Combining these results yields a simple spectral function:

$$
\rho(E)=\delta\left(E-E_{1}\right)+\theta\left(E-E_{1}\right) \frac{\bar{\rho}_{0}}{\sqrt{\bar{\rho}_{0}\left(E-E_{1}\right)}} I_{1}\left(2 \sqrt{\bar{\rho}_{0}\left(E-E_{1}\right)}\right) .
$$

where $I_{1}$ is a modified Bessel function.

We observe that eq. (5.14) exhibits the same form as found in [49] from general considerations of the entanglement spectrum for two-dimensional CFT's. Let us review their analysis. In equation (5.7), one could consider the Rényi entropy as related to the Laplace transform of the spectral function $d(\lambda)$, with $q$ acting as the Laplace parameter. i.e.,

$$
e^{(1-q) S_{q}}=\int_{0}^{\lambda_{1}} d \lambda d(\lambda) \lambda^{q}=\int_{\mathcal{E}_{1}}^{\infty} d \mathcal{E} e^{-\mathcal{E}(q+1)} d\left(e^{-\mathcal{E}}\right)
$$

where we have made the change of variables $\lambda=e^{-\mathcal{E}}$. Note that comparing with (5.9), $\mathcal{E}$ is related to energy measured in the hyperbolic space by $\mathcal{E}=E / T_{0}+\ln Z\left(T_{0}\right)$, and $\mathcal{E}_{1}=E_{1} / T_{0}+\ln Z\left(T_{0}\right)$. Given $S_{q}$, therefore, one could consider inverting the expression via an inverse Laplace transform:

$$
d\left(e^{-\mathcal{E}}\right)=\frac{1}{2 \pi i} \int_{\gamma-i \infty}^{\gamma+i \infty} d q e^{(1-q) S_{q}} e^{q \mathcal{E}}
$$

where $\gamma$ is suitably chosen for the convergence of the integral. This has been considered in [49]. The complete spectral function for the Rényi entropy (2.24) is given by

$$
\begin{aligned}
d(\lambda) & =\delta\left(\lambda_{1}-\lambda\right)+b \frac{\theta\left(\lambda_{1}-\lambda\right)}{\lambda \phi} I_{1}(2 \phi), \\
\phi & =\sqrt{b \ln \left(\lambda_{1} / \lambda\right)}, \quad b=-\ln \lambda_{1} .
\end{aligned}
$$

Hence we can see that there is full agreement between this result and eq. (5.20).

In higher dimensions, one can consider pursuing the same exercise. However, given the increased complexity of the expression for $S_{q}$, we would satisfy ourselves with an asymptotic expression, where $\lambda=\exp (-\mathcal{E}) \ll 1$ or $\mathcal{E} \gg 1$. In which case, we can 
approximate the integral via the saddle-point approximation. The effective Lagrangian in the integral (5.22) for Einstein gravity is given by

$$
L=q \mathcal{E}-(q-1) S_{q}=q \mathcal{E}-\pi q V_{\Sigma}\left(\frac{\tilde{L}}{\ell_{\mathrm{P}}}\right)^{d-1}\left(2-x_{q}^{d-2}\left(x_{q}^{2}+1\right)\right) .
$$

The saddle point is difficult to solve for general $d$, but can be worked out easily for explicit values of $d$.

Checking the results for $d=4$, we find

$$
d\left(\lambda=e^{-\mathcal{E}}\right) \sim \sqrt{\pi 3^{1 / 8}} \frac{e^{2 \Lambda^{\frac{1}{4}}\left(\frac{\mathcal{E}}{3}\right)^{\frac{3}{4}}}}{2 \mathcal{E}^{\frac{5}{8}} \Lambda^{\frac{1}{8}}} .
$$

where we have $\Lambda=\pi V_{\Sigma}\left(\frac{\tilde{L}}{\ell_{\mathrm{P}}}\right)^{d-1}$. Combining the results at $d=2$, this fits into the pattern of

$$
d\left(\lambda=e^{-\mathcal{E}}\right) \sim \frac{\exp \left(\mathcal{E}^{(d-1) / d}\right)}{\mathcal{E}^{(d+1) / 2 d}} .
$$

The power dependence of $\mathcal{E}$ in the exponential is exactly what is expected of the spectral density of a conformal field theory in flat space. Since we are in some high energy limit, the background curvature becomes irrelevant which thus leads to the same behavior. It would be interesting to find out whether field theories in higher dimensions display the same degree of universality as in two dimensions 49.

\section{Discussion}

We have presented several new results in this paper. The first was an extension the approach of [9] for calculating entanglement entropy to a new approach to calculate the Rényi entropy of a general CFT in $d$ dimensions with a spherical entangling surface. This new calculation evaluates the Rényi entropy in terms of certain thermal partition functions of the CFT on the background $R \times H^{d-1}$. While this result is independent of holography, this calculation has a simple holographic translation to a gravitational calculation in the context of the AdS/CFT correspondence. Hence we applied this approach to calculate the Rényi entropy for variety of holographic models in section 2. Our results, e.g., in eqs. (2.38) and (2.56), indicate that the Rényi entropy in higher dimensions is a complicated nonlinear function of the central charges and other parameters which characterize the CFT. We emphasize that the latter result should be expected to apply beyond holographic CFT's. That is, since no simple universal formula appears in our holographic results, we should not expect any simplification in 
general. The fact that more data than the central charges enters the Rényi entropy for a spherical entangling surface in higher dimensions is perhaps not so surprising. Even in two dimensions, if we consider two separate intervals calculating the Rényi entropy requires detailed information about the full spectrum of the corresponding CFT [51].

In section 1 , we elucidated the relation between this new thermal calculation and a conventional calculation of the Rényi entropy where a twist operator is inserted on the spherical entangling surface. Here one begins with the thermal path integral on the Euclidean background $S^{1} \times H^{d-1}$ where the period of the circle is given by $q / T_{0}$. If we choose $q$ to be a positive integer, the Euclidean version of the conformal mapping employed in [9] takes this background to a $q$-fold cover of flat space $R^{d}$ with an orbifold singularity on a sphere of radius $R$ with an angular excess of $2 \pi(q-1)$. This relation also allowed us to calculate the conformal scaling dimension of the twist operator. The latter was expressed in terms of the energy density of the thermal ensemble in eq. (4.21) or in terms of the thermal entropy and the corresponding Rényi entropy in eq. (4.23). Again these results do not depend on holography, however, the analysis is easily translated to a gravitational calculation using the AdS/CFT correspondence. Hence we calculated $h_{q}$ for the various holographic models introduced in section 2. Again, in general, the result was found to be a complicated nonlinear expression written in terms of the central charges, as well as other parameters in the CFT. However, we observed a remarkable simplification upon considering the expression $\left.\partial_{q} h_{q}\right|_{q=1}$, which is given by simply the central charge $\widetilde{C}_{T}$ appearing in the two-point function of the stress tensor. We should add that our calculations here have a strong similarity to those appearing in [52]. The latter analyzed holographic conformal field theory in de Sitter space but at an arbitrary temperature which need not match that of the cosmological horizon.

An interesting consequence of eq. (1.10) is that all of the Rényi entropies calculated for a spherical entangling surface in a $d$-dimensional CFT exhibit the same divergence structure as the corresponding entanglement entropy. These UV divergences arise from the integral over the same (infinite) hyperbolic geometry in all cases and were encapsulated by the factor $V_{\Sigma}$ given in eq. (2.10). While in general the individual power law divergences are sensitive to the details of the regulator in the CFT, we also identified a universal contribution (2.11) in this expression. However, if one calculates all of the Rényi entropies with a certain fixed regulator, it should be that one can consider the ratio $S_{q} / S_{1}$ which remains finite in the limit $\delta \rightarrow 0$. In this limit, the ratio is determined by the coefficients of the leading power law divergent terms and hence their ratio yields universal information characterizing the CFT. However, since the entire regulator dependence of $S_{q}$ factors out as $V_{\Sigma}$, this ratio will not contain any new information aside from the coefficients already identified in the universal contributions in the individual $S_{q}$. 
While our results indicate that the Rényi entropy in higher dimensions is a complicated nonlinear function of the various parameters characterizing the CFT, it is interesting that when we explicitly plot $S_{q}$ as a function of, e.g., c/a in figure 2a or $t_{4}$ in figure $4 a$, the results appear to be essentially linear in these parameters over the physical regime. We examined the behaviour of $S_{q}$ in more detail for the holographic model with $d=4$ and GB gravity in section 2.2. There we observed that the linearity cannot continue to arbitrarily towards small $c / a$ because we must have $S_{q} / S_{1}<1$. However, it seems that the nonlinear terms are suppressed for large $c / a$ and in this regime, the linear approximation of $S_{q} / S_{1}$ is even better. One consequence is that eventually $S_{q} / S_{1}$ becomes negative for sufficiently large $c / a$, which is clearly unphysical! However, as we emphasized in our discussion in section 2.2, this unusal behaviour arises in a regime where we already know that the holographic model has unphysical properties, e.g., violations of causality. Behaviour similar to that observed for $d=4$ also appears for $d>4$ as well, as shown in figure 3. However, we note that for sufficiently large $d$ (i.e., $d>7)$ we find that $S_{q} / S_{1}$ can become negative within the physical regime defined in eq. (2.34). As we will discuss elsewhere [53, it seems that this unusual behaviour can be used to impose additional physical constraints on these holographic models with interesting consequences.

Another remarkable simplification was observed in section 4.1. There again, the scaling dimension $h_{q}$ in higher dimensions is found to be a complicated nonlinear function of the central charges and other parameters characterizing the CFT, however, in the limit $q \rightarrow 1, \partial_{q} h_{q}$ is given by a very simple expression (4.26) for all of our holographic models and it is precisely proportional to $\widetilde{C}_{T}$. In fact, the simplicity of this result strongly suggests that it should be a general result that applies beyond a holographic setting. That is, we conjecture that eq. (4.26) in fact holds for any CFT. Here we comment on a possible physical consequence of this result. Generally, any even dimensional surface operator will suffers from a Graham-Witten anomaly 54 and it has been observed that the anomaly controls how the vacuum expectation value of the operator scales under scaling transformations [41]. It is therefore a natural question to ask whether the Graham-Witten anomaly is related to the generalized scaling dimension $h_{q}$, when $d$ is even, for the twist operators appearing in the present discussion. In this context, the universal coefficient $\mathcal{C}$ of the logarithmic contribution to the entanglement entropy is given by precisely the Graham-Witten anomaly [21, 37. As explained in the sections 3 and 4 , the partition function $Z_{q}$ is given by the expectation value of a twist operator $\sigma_{q}$ inserted on the entangling surface. Hence we may write the Rényi entropy as $S_{q}=\log \left\langle\sigma_{q}\right\rangle /(1-q)$. Hence in the limit $q \rightarrow 1$, we identify the entanglement entropy as $S_{1}=-\left.\partial_{q} \log \left\langle\sigma_{q}\right\rangle\right|_{q=1}$. Therefore, the universal coefficient $\mathcal{C}$ is equal to the derivative with respect to $q$ of the corresponding coefficient in the expectation 
value of the twist operator in the same limit. At $d=2$, we can compare $\mathcal{C}$ with $\partial_{q} h_{q}$ at $q=1$ and they coincide. In higher even dimensions, for a spherical entangling surface in flat space, it is well known that $\mathcal{C}$ is proportional to the $A$-type central charge of the conformal field theory [21, 37]. However, our holographic results (4.26) suggests that $\left.\partial_{q} h_{q}\right|_{q \rightarrow 1}$ is given instead by $\widetilde{C}_{T}$, which controls the two-point function of the stress tensor, which is a distinct from the $A$-type central charge in higher dimensions. Hence it seems that there is no simple relation between the generalized conformal dimension and the Graham-Witten anomaly for twist operators in higher dimensions.

One important aspect of our holographic calculations of Rényi entropy was that the bulk geometries of which we make use are nonsingular. As described in sections 3 and 4 , the standard field theory calculation of Rényi entropy involves evaluating the partition function on a singular orbifold background. The naive holographic translation of this boundary calculation extends the orbifold singularity into the bulk spacetime [13]. However, the resulting bulk geometry does not satisfy the classical gravitational equations of motion precisely because of the presence of this singularity and so it seems that without a full understanding of string theory or quantum gravity in the bulk, we will not understand how to properly calculate with this bulk geometry. More importantly, it can be explicitly shown that the naive prescription suggested in 13 leads to incorrect results for the Rényi entropy in certain instances [7]. The key step in our approach was to find an alternative field theory calculation of the Rényi entropy involving a thermal partition function which had a simple holographic translation involving hyperbolic black holes in the bulk spacetime and of course, the Euclidean versions of these geometries are completely nonsingular. As stressed in section 3.3, one should be able to perform a holographic calculation of the Rényi entropy by choosing the boundary metric to match that on the orbifold appearing in the replica trick calculation and finding a new smooth bulk geometry which properly solves the gravity equations. In this approach, the conical singularities introduced by the twist operators would appear only in the induced metric on UV regulator surface. Of course, as shown in section 4, our thermal approach and the standard replica trick calculation are simply related by a conformal transformation. Hence in principle, the black hole geometries provide precisely the desired nonsingular bulk solution for the problem where the boundary metric matches that on the relevant orbifold. Of course, our holographic calculations only strictly apply for a spherical entangling surface and so calculating the Rényi entropy with more general entangling surfaces still presents a formidable challenge in constructing the appropriate smooth bulk geometry.

An interesting observation in [7] was that although prescription of [13] using singular bulk geometries to calculate $S_{q}$ led to incorrect results for general $q$, it still seems that the correct entanglement entropy results in the limit $q \rightarrow 1$. This correct result 
still relies on extending the boundary singularity into the bulk and so strictly speaking the bulk geometry does not satisfy the correct equations of motion. However we note that, in the $q \rightarrow 1$ limit, the deviation from the on-shell geometry is infinitesimal. A similar holographic calculation of the entanglement entropy in the case of a spherical entangling surface was presented in 21] where again an infinitesimal singularity appears in the bulk. However, following the analysis of [56], it was emphasized that the leading off-shell behaviour of this singularity is universal and hence the result for the entanglement entropy is reliable. In particular, the entanglement entropy requires expanding the gravity action only to linear order in the deviation from the on-shell value. However, this linear deviation is proportional to the gravitation equations of motion in the bulk. Hence the bulk term does not contribute and the non-trivial contribution comes exclusively from the boundary. It seems that similar reasoning may apply for the more general calculations in [13 so that the detailed extension of the singularity into the bulk is irrelevant to the final result for the entanglement entropy. However, it seems that one must still invoke some additional rotational symmetry about the bulk singularity to properly justify this approach. As noted in [21], an analogous result applies in calculations of black hole entropy where it is understood that the off-shell approach [55] will generally yields the correct Wald entropy [16] for stationary black hole solutions.

\section{Acknowledgments}

We would like to thank Matt Headrick, Patrick Hayden, Andreas Karch, Brian Swingle and Tadashi Takayanagi for useful conversations. Research at Perimeter Institute is supported by the Government of Canada through Industry Canada and by the Province of Ontario through the Ministry of Research \& Innovation. RCM also acknowledges support from an NSERC Discovery grant and funding from the Canadian Institute for Advanced Research. AY is also supported by a fellowship from the Natural Sciences and Engineering Research Council of Canada.

\section{A. Rényi entropy inequalities}

In information theory, the Rényi entropy refers to a probability distribution with $p_{i}>0$ and $\sum_{i} p_{i}=1$ [10]. It follows that $S_{q}$ must satisfy a variety of different inequalities 
[11]. In the following, we consider four such inequalities:

$$
\begin{aligned}
\frac{\partial S_{q}}{\partial q} & \leq 0 \\
\frac{\partial}{\partial q}\left(\frac{q-1}{q} S_{q}\right) & \geq 0 \\
\frac{\partial}{\partial q}\left((q-1) S_{q}\right) & \geq 0 \\
\frac{\partial^{2}}{\partial q^{2}}\left((q-1) S_{q}\right) & \leq 0 .
\end{aligned}
$$

In particular, we will show that these inequalities are naturally satisfied by the entropies found in our holographic calculations. Actually, these results do not rely on the holographic framework but rather they follow from the relation which we established in the introduction between the Rényi entropies for a spherical entangling surface and the thermal ensemble on the hyperbolic cylinder $R \times H^{d-1}$. Hence it follows that these inequalities are satisfied for any CFT, as long as the thermal ensemble is stable, as we will see below. First, let us recall the formula (1.10) relating the Rényi entropy and the thermal entropy,

$$
S_{q}=\frac{q}{q-1} \frac{1}{T_{0}} \int_{T_{0} / q}^{T_{0}} S_{\mathrm{th}}(T) d T,
$$

where $T_{0}=1 /(2 \pi R)$ as usual and $S_{\mathrm{th}}(T)$ denotes the thermal entropy on $R \times H^{d-1}$.

If we begin by considering eq. (A.2), the expression on the right-hand side yields

$$
\frac{\partial}{\partial q}\left(\frac{q-1}{q} S_{q}\right)=\frac{1}{q^{2}} S_{\mathrm{th}}\left(T_{0} / q\right) .
$$

Hence the inequality (A.2) follows simply because the thermal entropy is a positive quantity. Given that eq. (A.2) is satisfied, simple corollary that follows is $S_{q} \leq q /(q-$ 1) $S_{\infty}$ for $q>1$ [7]. However, we note that it seems that the basic physical property that $S_{\mathrm{th}}(T) \geq 0$ can provide a nontrivial constraint on some of the holographic models with higher curvatures — see the discussion in section 6 .

Next turning to eq. (A.4), the right-hand side yields

$$
\frac{\partial^{2}}{\partial q^{2}}\left((q-1) S_{q}\right)=-\left.\frac{T_{0}}{q^{3}} \frac{\partial S_{\mathrm{th}}}{\partial T}\right|_{T_{0} / q}=-\left.\frac{1}{q^{2}} \frac{\partial E_{\mathrm{th}}}{\partial T}\right|_{T_{0} / q}
$$

Hence this expression is proportional to the specific heat and it follows from the stability of the thermal ensemble that the specific heat must be positive. This stability then ensures that the inequality (A.3) is satisfied by the corresponding Rényi entropy. 
Using eq. (A.5), the relevant expression in the first inequality (A.1) becomes

$$
\frac{\partial S_{q}}{\partial q}=-\frac{1}{(q-1)^{2}} \frac{1}{T_{0}} \int_{T_{0} / q}^{T_{0}}\left[S_{\mathrm{th}}(T)-S_{\mathrm{th}}\left(T_{0} / q\right)\right] d T .
$$

The fact that the integrand here is positive for $q>1$ again follows from the stability requirement above. That is, a positive specific heat implies $\partial_{T} S_{\mathrm{th}}>0$ and hence the finite difference in the integrand above is also positive. Hence the desired inequality A.1) is again satisfied for $q>1$. Examining the case $0<q<1$ is facilitated by rewriting the above expression (A.8) as

$$
\frac{\partial S_{q}}{\partial q}=-\frac{1}{(1-q)^{2}} \frac{1}{T_{0}} \int_{T_{0}}^{T_{0} / q}\left[S_{\mathrm{th}}\left(T_{0} / q\right)-S_{\mathrm{th}}(T)\right] d T .
$$

Now it is clear that the same reasoning as above can be used to establish that eq. (A.1) is still satisfied for this range of $q$. Again a simple corollary that follows from eq. (A.1) is that $S_{n+m} \leq S_{n}$ for positive integers, $n$ and $m$.

Finally considering eq. (A.3), the right-hand side can be written as

$$
\frac{\partial}{\partial q}\left((q-1) S_{q}\right)=\frac{1}{T_{0}} \int_{T_{0} / q}^{T_{0}} S_{\mathrm{th}}(T) d T+\frac{1}{q} S_{\mathrm{th}}\left(T_{0} / q\right) .
$$

For $q>1$, the positivity of this expression readily follows simply because the thermal entropy is always a positive quantity and hence the corresponding inequality (A.3) holds. In considering the range $0<q<1$, it is better to write this expression as

$$
\frac{\partial}{\partial q}\left((q-1) S_{q}\right)=\frac{1}{T_{0}} \int_{T_{0}}^{T_{0} / q}\left[S_{\mathrm{th}}\left(T_{0} / q\right)-S_{\mathrm{th}}(T)\right] d T+S_{\mathrm{th}}\left(T_{0} / q\right) .
$$

In this case, the integral being positive follows from the stability of the thermal ensemble which implies $\partial_{T} S_{\mathrm{th}}>0$, as discussed above with eq. (A.8). Of course, the positivity of the last term in this expression again follows because the thermal entropy is positive. Hence the desired inequality also holds in the range $0<q<1$.

\section{B. Rényi entropy in $d=3$}

The results in section 2 indicate that the Rényi entropies with a spherical entangling surface for CFT's in higher dimensions do not have a simple form analogous to the two-dimensional result (1.5). In fact, for even dimensions, the Rényi entropies depend on CFT parameters beyond simply the central charges. The latter contrasts with the known result for the entanglement entropy in any dimension where the coefficient of the 
universal term is simply a linear combination of the central charges appearing in the trace anomaly [5, 21, 37, 81. The precise linear combination depends on the geometry and for a spherical entangling surface, it is simply proportional to $A$, the central charge in the A-type anomaly [34.

However, the holographic calculations leading to the above conclusion only refer to $d \geq 4$. Hence one might still hope for some kind of simplification for the form of the Rényi entropy in $d=3$. We would like to examine this question with a nontrivial holographic model with a four-dimensional bulk. The theory we analyze here is a higher curvature theory with an interaction which is cubic in the Weyl tensor. ${ }^{16}$ To be concrete, we consider the following action

$$
I_{C^{3}}=\frac{1}{2 \ell_{\mathrm{P}}^{2}} \int d^{4} x \sqrt{-g}\left(\frac{6}{L^{2}}+R+\gamma L^{4} C_{a b c d} C^{c d e f} C_{e f}^{a b}\right),
$$

While no exact analytic black hole solutions are known for this theory, we can readily construct solutions perturbatively in the (dimensionless) coupling $\gamma$. However, before doing so, let us consider the role of $\gamma$ in the dual boundary theory. Since the boundary is three-dimensional, there is no trace anomaly, however, we can still consider the effective central charges $C_{T}$ and $a_{d}^{*}$, as introduced in eq. (2.28). However, it turns out that because the cubic curvature interaction above is constructed with the Weyl tensor, neither of these parameters in the dual CFT is effected by $\gamma$. For example, $C_{T}$ is essentially determined by the graviton propagator in $\mathrm{AdS}_{4}$ space. However, since the Weyl curvature vanishes in AdS space, the cubic term in eq. (B.2) can not contribute to the graviton kinetic term in this background. As this reasoning suggests, the effect of the cubic interaction only begins to be felt with the introduction of a new interaction cubic in the gravitons. Therefore the new coupling is first seen in a modification of the parameters appearing in the three-point function of the boundary stress tensor. In fact, if we are working perturbatively in this coupling, one would find that to leading order $t_{4} \propto \gamma$ [38], where $t_{4}$ is the parameter introduced in eq. (2.49). Hence as a measure of simplicity, we wish to determine whether or not the Rényi entropies are independent of this additional CFT parameter $t_{4}$.

In the following analysis of the holographic Rényi entropy, we are working perturbatively in $\gamma$ and we will only present the relevant results to first order in this coupling. A useful check of any of the following expressions is that in the limit $\gamma \rightarrow 0$, they must

\footnotetext{
${ }^{16}$ Note that in four dimensions, the Weyl tensor is given by

$$
C_{a b c d}=R_{a b c d}-g_{a[c} R_{d] b}+g_{b[c} R_{d] a}+\frac{1}{3} R g_{a[c} g_{d] b} .
$$
}


reduce to the corresponding result for Einstein gravity with $d=3$, as given in section 2.1. Further note that since the Weyl tensor vanishes in AdS space, the new gravitational interaction does not effect the AdS curvature, i.e., as with Einstein gravity, $\tilde{L}=L$

In constructing the perturbative black hole solutions, we extend the metric ansatz (2.1) slightly to

$$
d s^{2}=-\left(\frac{r^{2}}{L^{2}} f(r)-1\right) N^{2}(r) d t^{2}+\frac{d r^{2}}{\frac{r^{2}}{L^{2}} f(r)-1}+r^{2} d \Sigma_{2}^{2}
$$

i.e., now $g_{t t}$ now contains an independent function $N(r)$. As before, $d \Sigma_{2}^{2}$ denotes the metric of a two-dimensional hyperbolic plane with unit curvature. As noted above, to zeroth order in $\gamma$, the solution is just the hyperbolic black hole solution for Einstein gravity presented in section (2.1) with $d=3$. The perturbative solution to linear order in $\gamma$ is then given by

$$
\begin{aligned}
& f(r)=1-\frac{\omega^{3}}{r^{3}}-\gamma \frac{2 \omega^{6}}{L^{2} r^{9}}\left(8 \omega^{3}+9 L^{2} r-12 r^{3}\right) \\
& N(r)=N_{0}\left(1-\gamma \frac{6 \omega^{6}}{r^{6}}\right), \quad N_{0}=\frac{L}{R}
\end{aligned}
$$

The constant $N_{0}$ is chosen so that the boundary metric matches that given in eq. (2.2). It is convenient to again parameterize the constant $\omega^{3}$ in terms of the horizon radius $r_{\mathrm{H}}$ which is still determined by eq. (2.3). Hence to first order in $\gamma$, we have

$$
\omega^{3}=r_{\mathrm{H}}^{3}-L^{2} r_{\mathrm{H}}+\gamma \frac{2\left(4 r_{\mathrm{H}}^{2}-L^{2}\right)\left(r_{\mathrm{H}}^{2}-L^{2}\right)^{2}}{r_{\mathrm{H}}^{3}} .
$$

The temperature is given by eq. (2.4) except that we should replace the constant $N$ with the function $N(r)$ (evaluated at $r=r_{\mathrm{H}}$ ). That is,

$$
T=\frac{N\left(r_{\mathrm{H}}\right)}{4 \pi}\left[\frac{2}{r_{\mathrm{H}}}+\left.\frac{r_{\mathrm{H}}^{2}}{L^{2}} \frac{\partial f(r)}{\partial r}\right|_{r=r_{\mathrm{H}}}\right] .
$$

To leading order in $\gamma$, we may write the temperature as

$$
T=\frac{1}{4 \pi R}\left(3 x-\frac{1}{x}+\gamma \frac{6\left(1-x^{2}\right)^{2}}{x^{3}}\right) .
$$

where as before $x=r_{\mathrm{H}} / L$.

Next we determine the horizon entropy using Wald's formula (2.5), which yields

$$
S=\frac{2 \pi}{\ell_{\mathrm{P}}^{2}} \int_{\text {horizon }} d^{2} x \sqrt{h}\left[1+\frac{3}{2}\left(C_{a b}^{c d} C_{c d}{ }^{e f} \hat{\varepsilon}^{a b} \hat{\varepsilon}_{e f}-2 C_{a b}{ }^{c d} C_{c d}^{e b} \hat{\varepsilon}^{a f} \hat{\varepsilon}_{e f}-\frac{2}{3} C_{a b c d} C^{a b c d}\right)\right] .
$$


Recall that $\hat{\varepsilon}_{a b}$ is the binormal to the horizon and in Lorentzian signature, $\hat{\varepsilon}_{a b} \hat{\varepsilon}^{a b}=-2$. Substituting the above solution into this expression and expressing the result in terms of $x$ yields

$$
S(x)=2 \pi V_{\Sigma} \frac{L^{2}}{\ell_{\mathrm{P}}^{2}}\left(x^{d-1}+6 \gamma \frac{\left(x^{2}-1\right)^{2}}{x^{2}}\right)
$$

to first order in $\gamma$.

Finally we apply eq. (2.6) to calculate the Rényi entropy and we find

$$
S_{q}=\frac{\pi q}{q-1} V_{\Sigma} \frac{L^{2}}{\ell_{\mathrm{P}}^{2}}\left(2-x_{q}\left(1+x_{q}^{2}\right)+2 \gamma \frac{\left(1-4 x_{q}^{2}\right)\left(1-x_{q}^{2}\right)^{2}}{x_{q}^{3}}\right),
$$

where again $x_{q}$ is the value of $x=r_{\mathrm{H}} / L$ when the temperature (B.7) is $T=T_{0} / q$. Working perturbatively in $\gamma$, we find

$$
x_{q}=\frac{1+\sqrt{1+3 q^{2}}}{3 q}-4 \gamma \frac{\left(\sqrt{1+3 q^{2}}-2\right)^{2}}{3 q \sqrt{1+3 q^{2}}} .
$$

Considering the limits $q \rightarrow 0,1$ and $\infty$, we find to leading order in $\gamma$

$$
\begin{aligned}
& \lim _{q \rightarrow 0} S_{q}=\pi V_{\Sigma} \frac{L^{2}}{\ell_{\mathrm{P}}^{2}} \frac{8}{27}(1+2 \gamma) \frac{1}{q^{2}}, \\
& \lim _{q \rightarrow 1} S_{q}=2 \pi V_{\Sigma} \frac{L^{2}}{\ell_{\mathrm{P}}^{2}}, \\
& \lim _{q \rightarrow \infty} S_{q}=2 \pi V_{\Sigma} \frac{L^{2}}{\ell_{\mathrm{P}}^{2}}\left(1-\frac{2}{3 \sqrt{3}}+\frac{8}{3 \sqrt{3}} \gamma\right) .
\end{aligned}
$$

Here we simply note that our perturbative expression (B.10) of $S_{q}$ depends on $\gamma$ and further this dependence persists above in the limits $q \rightarrow 0, \infty$. Hence, it does not seem that there is any simplification in the form of the Rényi entropy in $d=3$, compared to our results for $d \geq 4$.

The scaling weight $h_{q}$ of the twist operators in the dual boundary theory can again be calculated using eq. (4.23) which yields

$$
h_{q}=\frac{-2 \pi}{x^{2}\left(1-3 x^{2}\right)^{2}} \frac{L^{2}}{\ell_{\mathrm{P}}^{2}}\left(-2 \gamma+10 x^{2} \gamma-(1+26 \gamma) x^{4}+3(1+6 \gamma) x^{6}\right) \text {. }
$$

The $q \rightarrow 1$ limit of course has the same simple form as that found in section (4.1):

$$
\left.\partial_{q} h_{q}\right|_{q=1}=\pi \frac{L^{2}}{\ell_{\mathrm{P}}^{2}}
$$




\section{The on-shell bulk action for $d=2$}

In this appendix, we review some of the details of the holographic construction presented in [46] when applied in our holographic calculation of the Rényi entropy in section 3.2. First, we write the three-dimensional bulk metric in FG gauge as given in eq. (3.13), which we reproduce here

$$
d s^{2}=\frac{L^{2} d \rho^{2}}{4 \rho^{2}}+\frac{g_{a b}(\omega, \bar{\omega}, \rho) d x^{a} d x^{b}}{\rho} .
$$

As discussed in the main text, the bulk geometry is the pure $\operatorname{AdS}_{3}$ space for $d=2$. In this case, the curvature tensor can be written in terms of the bulk metrc $G$ as

$$
R_{\mu \nu \rho \sigma}=-\frac{1}{L^{2}}\left(G_{\mu \rho} G_{\nu \sigma}-G_{\mu \sigma} G_{\nu \rho}\right)
$$

where $L$ is the AdS curvature scale. Substituting the metric $G$ in eq. (C.1) into this expression implies that

$$
\partial_{\rho}^{3} g_{a b}(\omega, \bar{\omega}, \rho)=0
$$

and so the expansion (3.14) of $g_{a b}(\omega, \bar{\omega}, \rho)$ terminates at order $\rho^{2}$. In fact, the full result may be written as

$$
g=\left(1+\frac{\rho}{2} g_{(2)} g_{(0)}^{-1}\right) g_{(0)}\left(1+\frac{\rho}{2} g_{(0)}^{-1} g_{(2)}\right)
$$

In $d=2$, the conformal symmetry at the boundary alone does not completely determine $g_{(2)}$ since this is the order at which the expansion contains information about the state of the CFT, e.g., the vacuum expectation value of the energy-momentum tensor. Of course, in higher dimensions, this date enters the FG expansion at order $d$. In any event, the boundary conformal symmetry does fix the form of $g_{(2)}$ as follows

$$
g_{(2) a b}=-\frac{L^{2}}{2}\left(R_{(0)} g_{(0) a b}+\mathcal{T}_{a b}\right)
$$

where

$$
\nabla^{a} \mathcal{T}_{a b}=0, \quad \text { and } \quad g_{(0)}^{a b} \mathcal{T}_{a b}=-R_{(0)},
$$

with $R_{(0)}$, the Ricci scalar for the boundary metric $g_{(0)}$.

The symmetric tensor $\mathcal{T}_{a b}$ above behaves very much like a stress tensor. Hence, to generate solutions, 46] proposed to take the analogy more seriously, by introducing an auxiliary scalar Liouville field $\phi$ on the AdS boundary, whose action is

$$
I_{\phi}=\frac{1}{48 \pi} \int d \omega d \bar{\omega} \sqrt{g_{(0)}}\left(\frac{1}{2} g_{(0)}^{a b} \partial_{a} \phi \partial_{b} \phi-\phi R_{(0)}\right) .
$$


The resulting energy-momentum tensor, which we equate with the unknown tensor in eq. (C.5), is given by

$$
\mathcal{T}_{a b}=-\frac{1}{2} \partial_{a} \phi \partial_{b} \phi-\nabla_{a} \partial_{b} \phi+g_{(0) a b}\left(\frac{1}{4} g_{(0)}^{a b} \partial_{a} \phi \partial_{b} \phi+g_{(0)}^{a b} \nabla_{a} \partial_{b} \phi\right)
$$

where covariant derivatives $\nabla$ are again evaluated with $g_{(0)}$. With the introduction of $\phi$, the second expression in eq. (C.6) becomes

$$
g_{(0)}^{a b} \nabla_{a} \partial_{b} \phi=-R_{(0)} .
$$

We now focus on the specific case where the boundary is the universal cover of the orbifold, in which case $g_{(0)}$ is given by eq. (3.15). We know that the universal cover is not flat everywhere and its curvature can be deduced from the conformal transformation (3.5) that takes us to the flat z-plane, i.e.,

$$
R_{(0)}\left[g_{z \bar{z}}\right]=\Omega^{-2}\left(R_{(0)}\left[g_{\omega \bar{\omega}}\right]-4 g^{\omega \bar{\omega}} \partial_{\omega} \partial_{\bar{\omega}} \ln \Omega\right)=0
$$

where

$$
\Omega=\left|\left(\omega-v_{1}\right)^{-(1-1 / q)}\left(\omega-v_{2}\right)^{-(1+1 / q)}\right| .
$$

Substituting this expression into eq. (C.9) then yields

$$
\bar{\partial}_{\omega} \partial_{\omega} \phi=-2 \bar{\partial}_{\omega} \partial_{\omega} \ln \Omega
$$

which is readily solved with

$$
\phi=-2 \ln \Omega+F(\omega)+\overline{F(\omega)}
$$

where $F(\omega)$ is some general holomorphic function. For simplicity, let us set $F(\omega)=0$ and as we will see this is the correct choice given the conformal transformation (3.4) which we performed at the boundary. We may simplify eq. (C.8) for the boundary metric $g_{(0)}$ given by eq. (3.15) to find

$$
\mathcal{T}_{\omega \omega}=-\partial \partial \phi-\frac{1}{2}(\partial \phi)^{2}=\overline{\left(\mathcal{T}_{\bar{\omega} \bar{\omega}}\right)}, \quad \mathcal{T}_{\omega \bar{\omega}}=\partial \bar{\partial} \phi
$$

Substituting the explicit solution (C.13) (with $F=0$ ) into the above expression then yields

$$
\mathcal{T}_{\omega \omega}=\frac{1}{2}\left(1-\frac{1}{q^{2}}\right) \frac{\left(v_{2}-v_{1}\right)^{2}}{\left(\omega-v_{2}\right)^{2}\left(\omega-v_{1}\right)^{2}} .
$$


Of course, the tensor $\mathcal{T}_{a b}$ is simply to the expectation value of the stress tensor $T_{a b}$ in the boundary theory. With $d=2$, the relation is found to be ${ }^{17}$

$$
\left\langle T_{a b}\right\rangle=\frac{1}{L \ell_{\mathrm{P}}}\left(g_{(2) a b}-g_{(0) a b} \operatorname{Tr}\left(g_{(0)}^{-1} g_{(2)}\right)\right)=-\frac{1}{2} \frac{L}{\ell_{\mathrm{P}}} \mathcal{T}_{a b} .
$$

Here the final expression comes from substituting eqs. (C.5) and (C.6) for $g_{(2)}$. Hence combining eq. (C.15) with the usual result for the central charge $c=12 \pi L / \ell_{\mathrm{P}}$ (for Einstein gravity in the bulk), we arrive at

$$
\left\langle T_{\omega \omega}\right\rangle=-\frac{c}{48 \pi}\left(1-\frac{1}{q^{2}}\right) \frac{\left(v_{2}-v_{1}\right)^{2}}{\left(\omega-v_{2}\right)^{2}\left(\omega-v_{1}\right)^{2}},
$$

which precisely matches the CFT result (3.7).

Perhaps this result should not be surprising since it is well known that a Weyl rescaling will shift the action of any $d=2$ CFT action by an expression proportional to the Liouville action, where the Liouville field takes the value of the Weyl factor [58]. Further, in the case where the Weyl factor is generated by a conformal transformation, the stress tensor of the Liouville action evaluates precisely to the Schwarzian. The holographic result suggests that the bulk gravitational action should also be shifted by the same Liouville action (as a surface term) relative to action evaluated in Poincaré coordinates. ${ }^{18}$

Nevertheless, to proceed with our holographic calculation of the Rényi entropy, we begin by substituting the metric (C.4) into the gravitational action and evaluating it on-shell. The latter action consists of the Einstein-Hilbert action $I_{E H}$ and two boundary contributions, the Gibbons-Hawking term $I_{G H}$ and a counter-term action $I_{c t}$ [22]:

$$
\begin{array}{cl}
I_{\mathrm{tot}}=\frac{1}{2 \ell_{\mathrm{P}}}\left(I_{E H}+I_{G H}+I_{c t}\right), & I_{E H}=-\int d^{3} x \sqrt{G}\left(R+\frac{2}{L^{2}}\right), \\
I_{G H}=-2 \int_{U V} d^{2} x \sqrt{h} K, & I_{c t}=\frac{2}{L} \int_{U V} d^{2} x \sqrt{h} .
\end{array}
$$

Above the two boundary contributions are evaluated on the UV regulator surface $\rho=$ $\rho_{\text {min }}=\delta^{2} / L^{2}$, where $\delta$ is the short distance cut-off in the boundary theory which was introduced in the main text. In particular then, $h(\omega, \bar{\omega})$ and $K$ are respectively the induced metric and the extrinsic curvature on this regulator surface.

\footnotetext{
${ }^{17} \mathrm{We}$ comment here that the definitions of the energy-momentum tensor in [2] and [46] differ by the same factor of $-2 \pi$ that was noted in footnote 11 .

${ }^{18}$ This need not seem obvious at this point. However, we return to addressing this issue in section 3.3 .
} 
Let us begin with $I_{E H}$. Since the bulk geometry is pure $\mathrm{AdS}_{3}$ space, this contribution reduces to

$$
I_{E H}=\int d \rho d \omega d \bar{\omega} \sqrt{\operatorname{det} g(\rho, \omega, \bar{\omega})} \frac{2}{\rho^{2} L^{2}} .
$$

Now using eq. (C.4), we may evaluate $\operatorname{det} g$ as

$$
\operatorname{det}[g]=\operatorname{det}\left[1+\frac{\rho}{2} g_{(2)} g_{(0)}^{-1}\right] \operatorname{det}\left[g_{(0)}\right] \operatorname{det}\left[1+\frac{\rho}{2} g_{(0)}^{-1} g_{(2)}\right] .
$$

Since the matrix $\left(1+\frac{\rho}{2} g_{(2)} g_{(0)}^{-1}\right)$ is the transpose of $\left(1+\frac{\rho}{2} g_{(0)}^{-1} g_{(2)}\right)$, the corresponding determinants are the same. Therefore we have

$$
\sqrt{\operatorname{det} g}=\sqrt{\operatorname{det} g_{(0)}} \operatorname{det}\left(1+\frac{\rho}{2} g_{(2)} g_{(0)}^{-1}\right) .
$$

In the bulk action, $\rho$ is integrated from the UV cut-off $\rho_{\min }$ to some upper bound $\rho_{\text {crit }}$. In [46], it was suggested that a natural upper bound would be $\operatorname{det}[g]$ becomes zero, which can be interpreted as the center of bulk space. Taking this approach, we must solve

$$
\left.\operatorname{det}\left(1+\frac{\rho}{2} g_{(2)} g_{(0)}^{-1}\right)\right|_{\rho_{\min }}=0
$$

which, using eq. (C.6), yields

$$
\rho_{\text {crit }}^{ \pm}=\frac{8}{L^{2}\left(R_{(0)} \mp 4 \sqrt{\Delta}\right)}, \quad \text { with } \quad \Delta=\frac{1}{8}\left(\operatorname{tr}\left(\mathcal{T}^{2}\right)-\frac{1}{2}(\operatorname{tr} \mathcal{T})^{2}\right) .
$$

Above, the traces are again taken using the boundary metric $g_{(0)}$. The appropriate physical solution is the smaller solution $\rho_{\text {crit }}^{-}$. Finally, let us note that in these coordinates, the surface terms $I_{G H}+I_{c t}$ cancel out all of the power law divergences in the bulk term $I_{E H}$. Explicitly, we have

$$
\begin{aligned}
I_{c t} & =2 L \int d \omega d \bar{\omega} \frac{\sqrt{\operatorname{det} g(\delta)}}{\delta^{2}}=\frac{2 L}{\delta^{2}} \int d \omega d \bar{\omega} \sqrt{\operatorname{det} g_{(0)}}\left(1-\frac{\delta^{2}}{4} R_{(0)}\right) \\
I_{G H} & =L \int d \omega d \bar{\omega} \frac{\sqrt{\operatorname{det} g(\delta)}}{\delta^{2}}\left(-4+2 \delta^{2} g^{\mu \nu}(\delta) g_{\mu \nu}^{\prime}(\delta)\right)=-\frac{4 L}{\delta^{2}} \int d \omega d \bar{\omega} \sqrt{\operatorname{det} g_{(0)}} .
\end{aligned}
$$

Hence by combining the above expressions, the on-shell gravity action (C.18) becomes

$$
I_{\text {tot }}=-\frac{c}{12 \pi L^{2}} \int d \omega d \bar{\omega} \sqrt{g_{(0)}}\left[\frac{L^{2}}{4} R_{(0)} \ln \rho_{\text {crit }}^{-}+\frac{1}{\rho_{\text {crit }}^{-}}+\frac{L^{2}}{4} R_{(0)}\right],
$$

One further simplification follows if we substitute the expression for $\rho_{\text {crit }}^{-}$given in eq. (C.23) into the second term in the integrand above:

$$
I_{\text {tot }}=-\frac{c}{12 \pi} \int d \omega d \bar{\omega} \sqrt{g_{(0)}}\left[L^{2} \frac{R_{(0)}}{4} \ln \rho_{\text {crit }}^{-}+\frac{1}{2} \sqrt{\left|\mathcal{T}_{\omega \omega}\right|^{2}}+\frac{3}{8} L^{2} R_{(0)}\right] .
$$


We use this result to evaluate the Rényi entropy of the boundary CFT in section 3.2.

As noted in section 3.3, the primary role of the FG coordinates is to select an interesting UV regulator surface in our holographic calculation of the Rényi entropy. That is, with $d=2$, the bulk geometry is empty $\mathrm{AdS}_{3}$ space and so the interesting physics comes from the 'unusual' asymptotic regulator surface whose choice is motivated by the problem of calculating the Rényi entropy. Let us examine the geometry of this regulator surface in more detail here. First we note that the coordinate transformation that takes us from general FG coordinates (C.1) to the standard Poincaré coordinates coordinates,

$$
d s^{2}=\frac{L^{2}}{\xi^{2}}\left(d \xi^{2}+d z d \bar{z}\right)
$$

for an $\mathrm{AdS}_{3}$ bulk was found by [47. Applying this result to the present case, we find

$$
\xi=\frac{\rho^{1 / 2} e^{-\hat{\phi}}}{1+L^{2} \rho e^{-2 \hat{\phi}}\left|\partial_{y} \hat{\phi}\right|^{2}} \quad z=y+\frac{L^{2} \rho e^{-2 \hat{\phi}} \partial_{\bar{y}} \hat{\phi}}{1+L^{2} \rho e^{-2 \hat{\phi}}\left|\partial_{y} \hat{\phi}\right|^{2}}
$$

where

$$
y \equiv\left(\frac{\omega-v_{1}}{\omega-v_{2}}\right)^{\frac{1}{q}}
$$

Further $\hat{\phi}$ is related to the conformal factor appearing in eq. (3.5),

$$
e^{\hat{\phi}} \equiv \frac{q}{v_{2}-v_{1}}\left|\omega-v_{1}\right|^{(1-1 / q)}\left|\omega-v_{2}\right|^{(1+1 / q)}=q\left(v_{2}-v_{1}\right) \frac{|y|^{q-1}}{\left|y^{q}-1\right|^{2}} .
$$

We observe that in the asymptotic limit, $\rho \rightarrow 0$, the second expression in eq. (C.28) becomes simply $z=y$. Hence on the boundary, this transformation reduces to precisely that between the $\omega$-plane and its universal cover given in eq. (3.4).

Now the region that is being cut out by our regularization in eq. (3.17) can easily be understood using this coordinate transformation. The constant factors can be readily absorbed by a rescaling of the coordinates and will be ignored below. Further we will set $L=1$ to avoid clutter in the following. We begin by manipulating the above transformation (C.28) by taking ratios of the two expressions to solve for $\rho e^{-2 \hat{\phi}}$ and then substituting the result back in to the equation for $z$ to obtain

$$
\frac{|z-y|}{\xi^{2}+|z-y|^{2}}=\left|\partial_{y} \hat{\phi}\right|
$$

Now for illustrative purposes, let us focus on $\omega \simeq v_{1}$. In the analysis of the onshell action above the integration over the boundary coordinates was regulated here by cutting off the integral at $\left|\omega-v_{1}\right|=\delta$ - see eq. (3.17). Alternatively, using eq. (C.29), 
we can think that this cut-off surface was placed at $y \simeq \delta^{1 / q} /\left(v_{2}-v_{1}\right) \equiv \hat{\delta}$. Now from eq. (C.30), in the vicinity of $y=0$, we have

$$
e^{\hat{\phi}} \simeq q\left(v_{2}-v_{1}\right)|y|^{q-1}
$$

Combining these results in eq. (C.31) then yields

$$
\xi^{2}+|z-\hat{\delta}|^{2} \simeq 2 \frac{\hat{\delta}}{q-1}|z-\hat{\delta}| .
$$

To simplify the discussion, let us consider the cross-section of this surface given by fixing $z$ to be real (and positive). Then eq. (C.33) describes a semi-circle centered on the $\xi$-axis at $z=\hat{\delta} q /(q-1)$ and with radius $\hat{\delta} /(q-1)$ - see figure 6. Hence the semi-circle reaches the asymptotic boundary, i.e., $\xi=0$, at $z=\hat{\delta}$ and $\hat{\delta}(q+1) /(q-1)$. However, let us consider general curves of constant $y$ in the vicinity of $y \simeq 0$ :

$$
\xi^{2}+(z-y)^{2} \simeq 2 \frac{y}{q-1}(z-y)
$$

where we are assuming that both $z$ and $y$ are real and positive. Treating $y$ as a parameter, eq. (C.34) describes a semi-circle in the $(\xi, z)$-plane, which is now centered on the $\xi$-axis at $z=y q /(q-1)$ and has radius $y /(q-1)$. This may seem slightly confusing since if we consider some value of $y$ slightly larger than the cut-off $y=\hat{\delta}$, we find the corresponding surface is slightly larger semi-circle which has moved to the right, as illustrated in figure 6. In particular, this constant $y$ curve crosses the cut-off surface described by eq. (C.33). This reflects a degeneracy in the coordinates and we will argue below that we should only consider the left-hand (blue) portion of any of these semi-circles.

Recall in our evaluation of the on-shell action above, the integration over $\rho$ is bounded in the IR by $\rho_{\text {crit }}^{-}$, as given in eq. (C.23). Let us examine this bound in the region $\omega \simeq v_{1}$ or $y \simeq 0$. First we observe that with $y \geq \hat{\delta}$ we stay away from the singularity in the boundary metric and so $R_{(0)}=0$. In this case, eq. (C.23) reduces to $\rho_{\text {crit }}^{-}=2 /\left|\mathcal{T}_{\omega \omega}\right|$. Then using eq. C.15), we find in the vicinity of $y \simeq 0$ that the IR boundary becomes

$$
\rho=\rho_{\text {crit }}^{-} \simeq \frac{4|y|^{2 q}\left(v_{2}-v_{1}\right)^{2}}{1-\frac{1}{q^{2}}} .
$$

We can express this equation in terms of $\xi$ and $z$ by substituting the above into eq. (C.28) and then eliminating $y$. The final result takes the simple form:

$$
\xi=\frac{z}{\sqrt{q^{2}-1}}
$$




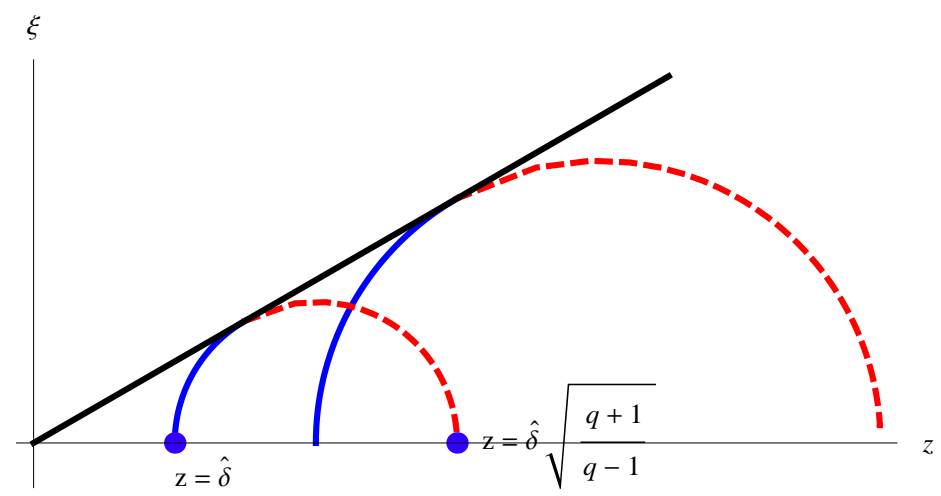

Figure 6: (Colour Online) FG coordinate surfaces near the singularity in the boundary metric at $\omega=v$ illustrated in Poincaré coordinates. The semi-circle to the left corresponds to the surface $y=\hat{\delta}$ given by eq. (C.33). The semi-circle to the right again corresponds to a constant $y$ surface for a slightly larger value of $y$. The black line corresponds to the IR boundary, i.e., $\rho=\rho_{\text {crit }}^{-}$. As discussed in the main text, the red dashed portions of the semi-circles are spurious.

That is, the IR boundary is simply a straight line with slope $1 / \sqrt{q^{2}-1}$ extending out from the origin in the $(\xi, z)$-plane. Examining this surface more carefully we find that it is precisely tangent to the constant $y$ semi-circles defined by eq. (C.34), as illustrated in figure 6. This feature is not a coincidence as $\rho_{\text {crit }}^{-}$in eq. (C.23) was chosen to correspond to the vanishing of $\operatorname{det}[g]$. We can now infer that, in the present case, this vanishing corresponds to a degeneracy of the FG coordinates, i.e., $d \rho \propto(d \omega+d \bar{\omega})$ along the surface $\rho=\rho_{\text {crit }}^{-}$. In any event, this analysis also indicates that the part of the semi-circles (C.34) that bends downwards as $y$ increases, is actually excluded in the coordinate patch covered by $\rho$ and $y$. That is, we should only consider the left-hand (blue) portion of any of the semi-circles illustrated in figure 6 .

Of course, a similar analysis will reveal analogous behaviour in the vicinity of the singularity in the boundary metric at $\omega \simeq v_{2}$, which corresponds to the bulk region near $\xi=0, z \rightarrow \infty$. In particular then, the regulator $\left|\omega-v_{2}\right|>\delta$ corresponds to a cut-off surface that extends from the asymptotic boundary at $\xi=0$ to the IR boundary at $\rho=\rho_{\text {crit }}^{-}$. So far we have only described the portion of the cut-off surface in the bulk that appears in the vicinity of the singularities in the universal cover of the $\omega$-plane, i.e., near the insertions of the twist operators. The cut-off surface that completes the regulation of the bulk integrals has a more conventional form. Namely, it is defined by asymptotic surface at $\rho_{\min }=\delta^{2}=\left(v_{2}-v_{1}\right)^{2 q} \hat{\delta}^{2 q}$. Hence the full cut-off surface can be regarded as having three components, the two surfaces cutting off the approach to the twist operators at $\omega=v_{2}$ and $\omega=v_{1}$, as well as a conventional UV cut-off surface 
extending over the rest of the boundary away from these singular points.

To close, let us comment on the difference between the various calculations of the on-shell action for $d=2$. In particular, above we considered a calculation based on choosing FG coordinates, while that proposed in section 3.3 relies directly on choosing an unusual regulator surface in the $\mathrm{AdS}_{3}$ vacuum. Similarly as described in the latter section, calculating the Euclidean action for the three-dimensional black hole (4.10) has a similar flavour of choosing an unconventional cut-off surface. However, a feature which distinguishes the first calculation is the appearance of an 'IR boundary' at $\rho=\rho_{\text {crit }}^{-}$. Again, the motivation for choosing this upper bound on the $\rho$ integration was to identify the center of the bulk geometry with the vanishing of $\operatorname{det}[g]$. However, as we can see in the discussion above, e.g., in figure 6, this interpretation fails in the present calculation. Rather this IR boundary simply corresponds to a degeneracy in the FG coordinate system and is not distinguished by any invariant property of the bulk geometry. A consequence of employing this upper bound on the $\rho$ integration is that a 'large' (but finite) part of the $\mathrm{AdS}_{3}$ bulk is simply excluded in evaluating the on-shell action. On the other hand, we have explicitly seen that both this calculation and the black hole calculation reproduce the correct Rényi entropy (1.5), expected for a two-dimensional CFT. In fact, eq. (1.5) corresponds to the universal contribution to the Rényi entropy and in general, we should expect that there may also be a non-universal constant contribution [2, 3]. Hence the difference between the various holographic calculations must lie in this non-universal contribution. In particular, the integration over the additional region in the bulk space beyond $\rho=\rho_{\text {crit }}^{-}$must only contribute a finite $\delta$-independent term. Hence this actually provides a reassuring demonstration that, within the holographic approach, different regulation schemes lead to the same universal contribution, as expected.

\section{References}

[1] See, for example:

M. Levin and X. G. Wen, "Detecting Topological Order in a Ground State Wave

Function," Phys. Rev. Lett. 96, 110405 (2006) [arXiv:cond-mat/0510613];

A. Kitaev and J. Preskill, "Topological entanglement entropy," Phys. Rev. Lett. 96, 110404 (2006) [arXiv:hep-th/0510092];

B. Hsu, M. Mulligan, E. Fradkin and E.A. Kim, "Universal entanglement entropy in 2D conformal quantum critical points," Phys. Rev. B 79, 115421 (2009) [arXiv:0812.0203].

[2] P. Calabrese and J. L. Cardy, "Entanglement entropy and quantum field theory," J.

Stat. Mech. 0406, P002 (2004) [arXiv:hep-th/0405152]. 
[3] P. Calabrese and J. L. Cardy, "Entanglement entropy and quantum field theory: A non-technical introduction," Int. J. Quant. Inf. 4, 429 (2006) [arXiv:quant-ph/0505193]; P. Calabrese and J. Cardy, "Entanglement entropy and conformal field theory," J. Phys. A 42 (2009) 504005 [arXiv:0905.4013 [cond-mat.stat-mech]].

[4] S. Ryu and T. Takayanagi, "Holographic derivation of entanglement entropy from AdS/CFT," Phys. Rev. Lett. 96, 181602 (2006) [arXiv:hep-th/0603001].

[5] S. Ryu and T. Takayanagi, "Aspects of holographic entanglement entropy," JHEP 0608, 045 (2006) [arXiv:hep-th/0605073].

[6] T. Nishioka, S. Ryu and T. Takayanagi, "Holographic Entanglement Entropy: An Overview," J. Phys. A 42, 504008 (2009) [arXiv:0905.0932 [hep-th]].

[7] M. Headrick, "Entanglement Renyi entropies in holographic theories," Phys. Rev. D 82, 126010 (2010) [arXiv:1006.0047 [hep-th]].

[8] L. Y. Hung, R. C. Myers and M. Smolkin, "On Holographic Entanglement Entropy and Higher Curvature Gravity," JHEP 1104, 025 (2011) [arXiv:1101.5813 [hep-th]].

[9] H. Casini, M. Huerta and R. C. Myers, "Towards a derivation of holographic entanglement entropy," arXiv:1102.0440 [hep-th].

[10] A. Rényi, "On measures of information and entropy," in Proceedings of the 4th Berkeley Symposium on Mathematics, Statistics and Probability, 1, 547 (U. of California Press, Berkeley, CA, 1961);

A. Rényi, "On the foundations of information theory," Rev. Int. Stat. Inst. 33 (1965) 1.

[11] For example, see:

K. Zyczkowski, "Renyi extrapolation of Shannon entropy," Open Syst. Inf. Dyn. 10, 297 (2003) [arXiv:quant-ph/0305062];

C. Beck and F. Schlögl, "Thermodynamics of chaotic systems", (Cambridge University Press, Cambridge, 1993).

[12] See, for example:

H. Li and F. D. M. Haldane, "Entanglement Spectrum as a Generalization of Entanglement Entropy: Identification of Topological Order in Non-Abelian Fractional Quantum Hall Effect States," Phys. Rev. Lett. 101, 010504 (2008) [arXiv:0805.0332 [cond-mat.mes-hall]]; S. T. Flammia, A. Hamma, T. L. Hughes, and X.-G. Wen, Phys. Rev. Lett. 103, 261601 (2009) [arXiv:0909.3305 [cond-mat.str-el]];

M. A. Metlitski, C. A. Fuertes, and S. Sachdev, Phys. Rev. B 80, 115122 (2009) [arXiv:0904.4477 [cond-mat.stat-mech]]; 
M. B. Hastings, I. Gonzalez, A. B. Kallin, and R. G. Melko, "Measuring Renyi

Entanglement Entropy in Quantum Monte Carlo Simulations," Phys. Rev. Lett. 104, 157201 (2010) [arXiv:1001.2335 [cond-mat.str-el]].

[13] D. V. Fursaev, "Proof of the holographic formula for entanglement entropy," JHEP 0609, 018 (2006) [arXiv:hep-th/0606184].

[14] G. Michalogiorgakis, "Entanglement entropy of two dimensional systems and holography," JHEP 0812, 068 (2008) [arXiv:0806.2661 [hep-th]].

[15] See, for example:

S. Aminneborg, I. Bengtsson, S. Holst and P. Peldan, "Making Anti-de Sitter Black

Holes," Class. Quant. Grav. 13, 2707 (1996) [arXiv:gr-qc/9604005];

D. R. Brill, J. Louko and P. Peldan, "Thermodynamics of (3+1)-dimensional black holes with toroidal or higher genus horizons," Phys. Rev. D 56, 3600 (1997)

[arXiv:gr-qc/9705012];

L. Vanzo, "Black holes with unusual topology," Phys. Rev. D 56, 6475 (1997) [arXiv:gr-qc/9705004];

R. B. Mann, "Pair production of topological anti-de Sitter black holes," Class. Quant. Grav. 14, L109 (1997) [arXiv:gr-qc/9607071];

D. Birmingham, "Topological black holes in anti-de Sitter space," Class. Quant. Grav. 16, 1197 (1999) [arXiv:hep-th/9808032]. R. Emparan, "AdS membranes wrapped on surfaces of arbitrary genus," Phys. Lett. B 432, 74 (1998) [arXiv:hep-th/9804031].

[16] R. M. Wald, "Black hole entropy is the Noether charge," Phys. Rev. D 48, 3427 (1993) [arXiv:gr-qc/9307038];

T. Jacobson, G. Kang and R. C. Myers, "On Black Hole Entropy," Phys. Rev. D 49, 6587 (1994) [arXiv:gr-qc/9312023];

V. Iyer and R. M. Wald, "Some properties of Noether charge and a proposal for dynamical black hole entropy," Phys. Rev. D 50, 846 (1994) [arXiv:gr-qc/9403028].

[17] J. C. Baez, "Renyi Entropy and Free Energy," arXiv:1102.2098 [quant-ph].

[18] D. Lovelock, "The Einstein tensor and its generalizations," J. Math. Phys. 12, 498 (1971);

D. Lovelock, "Divergence-free tensorial concomitants," Aequationes Math. 4, 127 (1970).

[19] R. C. Myers and B. Robinson, "Black Holes in Quasi-topological Gravity," JHEP 1008, 067 (2010) [arXiv:1003.5357 [gr-qc]].

[20] R. C. Myers, M. F. Paulos and A. Sinha, "Holographic studies of quasi-topological gravity," JHEP 1008, 035 (2010) [arXiv:1004.2055 [hep-th]]. 
[21] R. C. Myers and A. Sinha, "Holographic c-theorems in arbitrary dimensions," JHEP 1101, 125 (2011) [arXiv:1011.5819 [hep-th]];

R. C. Myers and A. Sinha, "Seeing a c-theorem with holography," Phys. Rev. D 82, 046006 (2010) [arXiv:1006.1263 [hep-th]].

[22] R. Emparan, C. V. Johnson and R. C. Myers, "Surface Terms as Counterterms in the AdS/CFT Correspondence," Phys. Rev. D60 (1999) 104001 [arXiv:hep-th/9903238]; S. de Haro, S. N. Solodukhin and K. Skenderis, "Holographic reconstruction of spacetime and renormalization in the AdS/CFT correspondence," Commun. Math. Phys. 217, 595 (2001) [arXiv:hep-th/0002230];

P. Kraus, F. Larsen and R. Siebelink, "The Gravitational Action in Asymptotically AdS and Flat Spacetimes," Nucl. Phys. B563 (1999) 259-278 [arXiv:hep-th/9906127];

A. Yale, "Simple counterterms for asymptotically AdS spacetimes in general theories of gravity," arXiv:1107.1250 [gr-qc].

[23] B. Hsu, M. Mulligan, E. Fradkin and E.A. Kim, "Universal entanglement entropy in 2D conformal quantum critical points," Phys. Rev. B 79, 115421 (2009) [arXiv:0812.0203].

[24] R.C. Myers and A. Yale, "A Holographic Calculation of Mutual Information," in preparation.

[25] H. Casini, "Mutual information challenges entropy bounds," Class. Quant. Grav. 24, 1293 (2007) [arXiv:gr-qc/0609126];

H. Casini and M. Huerta, "Remarks on the entanglement entropy for disconnected regions," JHEP 0903, 048 (2009) [arXiv:0812.1773 [hep-th]];

H. Casini and M. Huerta, "Entanglement entropy in free quantum field theory," J. Phys. A 42, 504007 (2009) [arXiv:0905.2562 [hep-th]].

[26] B. Swingle, "Mutual information and the structure of entanglement in quantum field theory," arXiv:1010.4038 [quant-ph].

[27] M. Henningson and K. Skenderis, "The holographic Weyl anomaly," JHEP 9807, 023 (1998) [arXiv:hep-th/9806087];

M. Henningson and K. Skenderis, "Holography and the Weyl anomaly," Fortsch. Phys. 48, 125 (2000) [arXiv:hep-th/9812032].

[28] A. Yale and T. Padmanabhan, "Structure of Lanczos-Lovelock Lagrangians in Critical Dimensions," Gen. Rel. Grav. 43 (2011) 1549-1570 [arXiv:arXiv:1008.5154 [gr-qc]]

[29] R. G. Cai, "Gauss-Bonnet black holes in AdS spaces," Phys. Rev. D 65 (2002) 084014 [arXiv:hep-th/0109133];

S. Nojiri and S. D. Odintsov, "Anti-de Sitter black hole thermodynamics in higher derivative gravity and new confining-deconfining phases in dual CFT," Phys. Lett. B 521 (2001) 87 [Erratum-ibid. B 542 (2002) 301] [arXiv:hep-th/0109122]; 
Y. M. Cho and I. P. Neupane, "Anti-de Sitter black holes, thermal phase transition and holography in higher curvature gravity," Phys. Rev. D 66 (2002) 024044 [arXiv:hep-th/0202140];

I. P. Neupane, "Black hole entropy in string-generated gravity models," Phys. Rev. D 67 (2003) 061501 [arXiv:hep-th/0212092];

I. P. Neupane, "Thermodynamic and gravitational instability on hyperbolic spaces," Phys. Rev. D 69 (2004) 084011 [arXiv:hep-th/0302132];

R. G. Cai, "A note on thermodynamics of black holes in Lovelock gravity," Phys. Lett. B 582, 237 (2004) [arXiv:hep-th/0311240].

[30] J. de Boer, M. Kulaxizi and A. Parnachev, "Holographic Entanglement Entropy in Lovelock Gravities," arXiv:1101.5781 [hep-th].

[31] M. Brigante, H. Liu, R. C. Myers, S. Shenker and S. Yaida, "Viscosity Bound Violation in Higher Derivative Gravity," Phys. Rev. D 77 (2008) 126006 [arXiv:htp-th/0712.0805];

M. Brigante, H. Liu, R. C. Myers, S. Shenker and S. Yaida, "The Viscosity Bound and Causality Violation," Phys. Rev. Lett. 100, 191601 (2008) [arXiv:0802.3318 [hep-th]]; A. Buchel and R. C. Myers, "Causality of Holographic Hydrodynamics," JHEP 0908, 016 (2009) [arXiv:0906.2922 [hep-th]];

D. M. Hofman, "Higher Derivative Gravity, Causality and Positivity of Energy in a UV complete QFT," Nucl. Phys. B 823, 174 (2009) [arXiv:0907.1625 [hep-th]];

X. H. Ge and S. J. Sin, "Shear viscosity, instability and the upper bound of the Gauss-Bonnet coupling constant," JHEP 0905, 051 (2009) [arXiv:0903.2527 [hep-th]];

R. G. Cai, Z. Y. Nie and Y. W. Sun, "Shear Viscosity from Effective Couplings of Gravitons," Phys. Rev. D 78, 126007 (2008) [arXiv:0811.1665 [hep-th]];

R. G. Cai, Z. Y. Nie, N. Ohta and Y. W. Sun, "Shear Viscosity from Gauss-Bonnet Gravity with a Dilaton Coupling," Phys. Rev. D 79, 066004 (2009) [arXiv:0901.1421 [hep-th]];

J. de Boer, M. Kulaxizi and A. Parnachev, "AdS $S_{7} / C F T_{6}$, Gauss-Bonnet Gravity, and Viscosity Bound," JHEP 1003, 087 (2010) [arXiv:0910.5347 [hep-th]];

X. O. Camanho and J. D. Edelstein, "Causality constraints in AdS/CFT from conformal collider physics and Gauss-Bonnet gravity," arXiv:0911.3160 [hep-th].

[32] S. Nojiri and S.D. Odintsov, "On the conformal anomaly from higher derivative gravity in AdS/CFT correspondence," Int. J. Mod. Phys. A 15, 413 (2000) [arXiv:hep-th/9903033];

M. Blau, K.S. Narain and E. Gava, "On subleading contributions to the AdS/CFT trace anomaly," JHEP 9909, 018 (1999) [arXiv:hep-th/9904179].

[33] A. Buchel, J. Escobedo, R. C. Myers, M. F. Paulos, A. Sinha and M. Smolkin, 
"Holographic GB gravity in arbitrary dimensions," JHEP 1003 (2010) 111 [arXiv:0911.4257 [hep-th]].

[34] S. Deser and A. Schwimmer, "Geometric classification of conformal anomalies in arbitrary dimensions," Phys. Lett. B309, 279-284 (1993) [hep-th/9302047].

[35] D. G. Boulware and S. Deser, "String Generated Gravity Models," Phys. Rev. Lett. 55 (1985) 2656.

[36] T. Jacobson and R. C. Myers, "Black Hole Entropy And Higher Curvature Interactions," Phys. Rev. Lett. 70, 3684 (1993) [arXiv:hep-th/9305016].

[37] S. N. Solodukhin, "Entanglement entropy, conformal invariance and extrinsic geometry," Phys. Lett. B 665, 305 (2008) [arXiv:0802.3117 [hep-th]].

[38] D. M. Hofman and J. Maldacena, "Conformal collider physics: Energy and charge correlations," JHEP 0805, 012 (2008) [arXiv:0803.1467 [hep-th]].

[39] J. Oliva and S. Ray, "A new cubic theory of gravity in five dimensions: Black hole, Birkhoff's theorem and C-function," Class. Quant. Grav. 27, 225002 (2010) [arXiv:1003.4773 [gr-qc]].

[40] H. Casini, "Entropy inequalities from reflection positivity," J. Stat. Mech. 1008, P08019 (2010) [arXiv:1004.4599 [quant-ph]].

[41] A. Kapustin, "Wilson-'t Hooft operators in four-dimensional gauge theories and S-duality," Phys. Rev. D74 (2006) 025005. [hep-th/0501015].

J. Gomis, S. Matsuura, T. Okuda, D. Trancanelli, "Wilson loop correlators at strong coupling: From matrices to bubbling geometries," JHEP 0808 (2008) 068. [arXiv:0807.3330 [hep-th]].

[42] G. Michalogiorgakis, "Entanglement entropy of two dimensional systems and holography," JHEP 0812 (2008) 068 [arXiv:0806.2661 [hep-th]].

[43] O. Lunin and S. D. Mathur, "Correlation functions for $M^{N} / S_{N}$ orbifolds," Commun. Math. Phys. 219 (2001) 399 [arXiv:hep-th/0006196].

[44] C. Fefferman and C. R. Graham, "Conformal Invariants," in Elie Cartan et les Mathématiques d'aujourd hui (Astérisque, 1985) 95;

C. Fefferman and C. R. Graham, "The Ambient Metric," arXiv:0710.0919 [math.DG].

[45] S. de Haro, S. N. Solodukhin and K. Skenderis, "Holographic reconstruction of spacetime and renormalization in the AdS/CFT correspondence," Commun. Math. Phys. 217, 595 (2001) [arXiv:hep-th/0002230]. 
[46] K. Skenderis and S. N. Solodukhin, "Quantum effective action from the AdS / CFT correspondence," Phys. Lett. B 472 (2000) 316 [arXiv:hep-th/9910023].

[47] K. Krasnov, "On holomorphic factorization in asymptotically AdS 3-D gravity," Class. Quant. Grav. 20 (2003) 4015 [arXiv:hep-th/0109198].

[48] For example, see:

S. Carlip, "The (2+1)-Dimensional black hole," Class. Quant. Grav. 12, 2853 (1995) [arXiv:gr-qc/9506079].

[49] P. Calabrese and A. Lefevre, "Entanglement spectrum in one-dimensional systems," Phys. Rev. A 78, 032329 (2008) arXiv:0806.3059 [cond-mat.str-el].

[50] R. C. Myers and M. Smolkin, in preparation.

[51] P. Calabrese, J. Cardy, E. Tonni, "Entanglement entropy of two disjoint intervals in conformal field theory," J. Stat. Mech. 0911, P11001 (2009) [arXiv:0905.2069 [hep-th]]; P. Calabrese, J. Cardy, E. Tonni, "Entanglement entropy of two disjoint intervals in conformal field theory II," J. Stat. Mech. 1101, P01021 (2011) [arXiv:1011.5482 [hep-th]].

[52] D. Marolf, M. Rangamani and M. Van Raamsdonk, "Holographic models of de Sitter QFTs," Class. Quant. Grav. 28 (2011) 105015 [arXiv:1007.3996 [hep-th]].

[53] L. Y. Hung, R. C. Myers, M. Smolkin and A. Yale, in preparation.

[54] C. R. Graham and E. Witten, "Conformal anomaly of submanifold observables in AdS/CFT correspondence," Nucl. Phys. B 546, 52 (1999) [arXiv:hep-th/9901021].

[55] C. G. . Callan and F. Wilczek, "On geometric entropy," Phys. Lett. B 333, 55 (1994) [arXiv:hep-th/9401072].

[56] D. V. Fursaev and S. N. Solodukhin, "On The Description Of The Riemannian Geometry In The Presence Of Conical Defects," Phys. Rev. D 52, 2133 (1995) [arXiv:hep-th/9501127].

[57] M. Banados, C. Teitelboim and J. Zanelli, "The Black hole in three-dimensional space-time," Phys. Rev. Lett. 69, 1849 (1992) [arXiv:hep-th/9204099].

[58] For example, see: D. Friedan, "Introduction To Polyakov's String Theory," in Recent Advances in Field Theory and Statistical Mechanices (Les Houches Summer School Proceedings, v. 39), eds., J.B. Zuber and R. Stora (Amsterdam, North-Holland, 1984. 871p.) 